\title{
The genus Eragrostis (Poaceae: Chloridoideae) in India: A Taxonomic Revision
}

\author{
Vivek, C.P*, G.V.S. Murthy ${ }^{1}$ and V.J. Nair'† \\ Botanical Survey of India, Andaman and Nicobar Regional Centre, Haddo Post \\ Port Blair - 744102, Andaman and Nicobar Islands, India \\ 'Botanical Survey of India, Southern Regional Centre, Lawley Road Post \\ Coimbatore - 641002, Tamil Nadu, India \\ *Corresponding author: vvkcpoulose@gmail.com

\section{भारत में वंश इराग्रोस्ट्रिस (पोएसी: क्लोरिडोईडी): एक वर्गीकीय पुनर्वलोकन}

विवेक सी.पी., जी.वी.एस. मूर्ती एवं वी.जे. नय्यर

\section{सारांश}

भारत में वंश इराग्रोस्ट्रिस वुल्फ के वर्गीकीय पुर्बरावलोकन में 42 जातियां एवं 5 प्रभेद शामिल हैं। इराग्रोस्ट्रिस टेनेल्ला प्रभेद पेरामंगलमेंसिस को इराग्रोस्ट्रिस टेनेल्ला प्रभेद टेनेल्ला, इराग्रोस्ट्रिस बर्मेनिका के अंतर्गत समानार्थ किया गया है। इस जाति का पता मणिपुर राज्य से लगाया गया है और इसे भारत के वनस्पतिजात में समाहित किया गया है। प्रस्तुत शोधपत्र में सभी जातियों एवं प्रभेदों का विवरण, सचित्र व्याख्या एवं वर्गिकी कुंजी सहित आकारिकी, वर्गिकी, नामकरण/ग्रंथीय सूची, ऋतुजैविकीय, सवितरण एवं प्रत्येक जाति की पारिस्थितिकीय विवरण दिया गया है।

\section{Abstract}

The taxonomic revision of the genus Eragrostis Wolf in India recognizes 42 species and 5 varieties. Eragrostis tenella var. peramangalamensis is synonymised under Eragrostis tenella var. tenella. Eragrostis burmanica is reported as an addition to India from the state of Manipur. The morphology, taxonomy, nomenclatural/bibliographic citations, phenology, distribution and ecology of every taxon are discussed along with descriptions, illustrations and key to all the species and varieties.

Keywords: Eragrostis, Grasses, India, Revision, Taxonomy

\section{INTRODUCTION}

Eragrostis Wolf (1776) is a morphologically diverse and taxonomically complicated genus in the subfamily Chloridoideae. The genus comprises approximately 423 species, and distributed in tropical, subtropical, and warm temperate regions of the world (Hartley \& Slater, 1960; Clayton \& Renvoize, 1986; Ingram \& Doyle,
2007; Ingram, 2010; Giraldo-Canas \& al., 2012). Of which 55 are native in Australia (Lazarides, 1997), 25 are native in the United States and Canada, and 36 are native in Mexico (Beetle \& al., 1991; Espejo-Serna \& al., 2000; Peterson \& al., 2001). The diagnostic features of the genus include ciliate ligules, paniculate inflorescence, rarely racemes or spikes and manyflowered (rarely 1-flowered) laterally compressed 
spikelets, the disarticulation of the lemma and palea occurs here separately, mainly disarticulating between the florets but also having other forms of disarticulation, glumes are usually 1-nerved, lemmas are usually 3nerved and unawned, the paleas are longitudinally bowed-out with ciliate, scaberulous or rarely smooth keels (Peterson \& al., 1997; Giraldo-Canas \& al., 2012). Eragrostis is notorious for its confusing infrageneric delimitation (Veldkamp, 2002; Ingram, 2010; GiraldoCanas \& al., 2012). The tribe is regarded as of an unnatural grouping of convenience, and Eragrostis itself as a large polyphyletic assemblage (Voigt \& al., 2004). Most of the species grow in open habitats with poor soils and many found in disturbed sites (Clayton \& Renvoize, 1986; Van den Borre \& Watson, 1994).

There are many taxonomic and systematic treatments on the genus Eragrostis. Some significant contributions are from Costa Rica (Pohl, 1980), Venezuela (Graterol \& al., 1989), Mexico (Beetle \& al., 1991; Peterson \& Valdes Reyna, 2005), Congo (Kami, 1993), Peru (Tovar, 1993; Peterson \& Vega Sanchez, 2007), Mesoamerica (Davidse, 1994), Australia (Lazarides, 1997), Argentina (Nicora, 1998), Zambesiaca (Cope, 1998), Benin (Houinato \& al., 2000), Brazil (Boechat \& Longhi-Wagner, 2000 \& 2001), France (Portal, 2002), Malesia (Veldkamp, 2002), the United States and Canada (Peterson, 2003), Colombia (Peterson, 2008), northwestern South America (Giraldo-Canas \& al., 2012), Thailand (Chaisongkrama \& al., 2013) etc. However, hitherto no such comprehensive taxonomic study has been carried out on the genus in India. Bor (1960) who provided an annotated list of the species of the genus in India and neighbouring countries reported 39 species from India. Karthikeyan $\&$ al. (1989) included 36 species from India. The recently published ckecklist of grasses of India (Kellogg \& al., 2020) included 51 taxa. Fischer (1934-1936), Cooke (1908), Haines (1921-1925), Roy (1976, 1984), Deshpande \& Singh (1986), Uniyal \& al. (1994), Lakshminarasimhan (1996), Shukla (1996), Moulik (1997), Daniel \& Umamaheshwari (2001) and Yadav \& Sardesai (2002) are some of the earlier workers who have included good number of Eragrostis species in thier respective Flora.

The present study describes 42 species and 5 varieties of Eragrostis that are distributed in different parts of
India. Many taxa have morphological variations and related problems in delimitaion. Some of them are looking alike owing to their resemblance in colour, size of the culms and spikelets due to which there are confusions in the identity and circumscription. There are inconsistencies in the descriptions provided in some of the earlier Floras. The present treatment is based on the study of herbarium specimens and live collections of Eragrostis in India and herbarium images from other countries including type specimens. This study contains a key for determining the species and varieties, nomenclatural/bibliographic citations, vernacular names of the species, if available, descriptions, phenology, habitat, distribution, taxonomic notes indicating inter-relationships of various taxa, specimens examined, and illustrations.

The morphological features of culms, leaves, panicle, spikelets and their components are used in the determination of species identity. The culms are usually erect to decumbent or geniculate at lower portions. The leaves are linear to lanceolate or flat with involute or folded margins. These characters are helpful for identification of Eragrostis in combination with other characters. The relative density of hairs and types of microhairs on leaves have some significance. In Indian Eragrostis, chloridoid and panicoid types of microhairs have been recorded (Vivek \& al., 2016). Ligule of most of the Eragrostis species in India is a fringe of cilia. Although the ligule is ciliate in most cases, in species such as E. gangetica (Roxb.) Steud., E. unioloides (Retz.) Nees ex Steud. and E. zeylanica Nees \& Mey.) it is narrowly membranous at base, but in E. japonica (Thunb.) Trin. the whole ligule is membranous. Morphology of panicle of a species is almost invariable. However, there are extreme variations in E. viscosa from open to spiciform conditions. Differences in the size of the panicles, length of pedicels and presence or absence of glands are reliable characters. The morphology of mature spikelets including shape, texture, size, in relation with number of florets and its mode of disarticulation from the rachilla are significant characters. Coulour of the spikelets is a supporting character, for instance the colour of spikelets of $E$. collinensis Vivek \& al. is deep reddish and that of $E$. nigra Nees ex Steud. is blackish green. In a number of species the florets disarticulation starts from base towards apex or in certain others it is from apex towards 
base. Rarely a mixed type of disarticulation is noticed in E. viscosa (Retz.) Trin. In E. superba Peyr., the glumes, lemmas and paleas are disarticulating as entire unit of spikelet from pedicels, but the spikelets do not disarticulate in E. tef (Zucc.) Trotter. Generally, the florests are closely arranged on rachilla, but relatively loosely arranged in a few species, are significant characters in species identification. The size, shape and texture of glumes, lemmas, paleas and caryopses are important diagnostic characters (Vivek \& al., 2015a, 2019). Lodicules, ovary, styles and stigmas are of less singnificance in the taxonomy of Eragrostis.

In India, Eragrostis are highly adapted to grow in different habitats from sea level to higher elevations, up to $2800 \mathrm{~m}$. Eragrostis collinensis, E. nilgiriensis Vivek \& al., E. nigra, E. schweinfurthii Chiov. and E. tenuifolia (A. Rich.) Hochst. ex Steud. are high-elevation species. Rest of them are distributed in different habitats such as riversides, seashores, plantations, agricultural fields, forest margins, wetlands, etc. Eragrostis unioloides has wide range of habitat adaptations from sea level up to $1000 \mathrm{~m}$ elevation. Eragrostis tenella var. insularis C.E. Hubb. is a costal taxon. Out of the 47 taxa, 8 are endemic to India. They are E. ciliaris var. clarkei Stapf (Delhi, Karnataka and Rajasthan), E. collinensis Vivek \& al. (Tamil Nadu), E. deccanensis Bor (Andhra Pradesh, Karnataka, Kerala, Odisha and Tamil Nadu), E. henryi Vivek \& al. (Tamil Nadu), E. jainii Vivek \& al. (Karnataka and Kerala), E. minor Host var. rajasthanensis Vivek \& al. (Rajasthan), E. nilgiriensis Vivek \& al. (Tamil Nadu), and E. tremula var. gajanandii Genda Singh \& al. (Rajasthan).

\section{MATERIALS AND METHODS}

The present study is based on the comprehensive investigation of specimens housed in various Indian herbaria such as BSA, BSD, BSHC, BSI, BSJO, CAL, CALI, FRC, MH, PBL, RHT, TBGT, Herbarium of Malabar Botanical Garden and Institute for Plant Sciences, Kozhikode, Kerala, and additional live collections and relevant literature. Information and images available in the databases of various national and international herbaria such as B, BM, BRI, E, FI, K, LD, L, LE, LINN, NTM, NY, P, UPS, W, and associated libraries were also studied. Live specimens of most of the species of Eragrostis could be collected, preserved and deposited in $\mathrm{MH}$. The species identity was confirmed mainly with the help of Stapf (1896), Bor (1960), Cope (1982), Moulik (1997), Sreekumar \& Nair (1991), Kabeer \& Nair (2009) and other important floras, revisions and monographs. Available type material of the species or digital images of types and protologues were consulted. Illustrations of habit and floral parts and images of spikelets using Nikon SMZ 1500 microscope of almost all the species were prepared for easy identification of key characters. The specimens consulted are cited under "specimens examined".

\section{TAXONOMIC TREATMENT}

Eragrostis Wolf, Gen. Pl.: 23. 1776.

Type: Eragrostis minor Host. Lectotype designated by Pfeiffer, Nomencl. Bot. 1(2): 1226. 1874-1875.

Annual or perennial. Culms (2)5-170 cm high, erect or decumbent, geniculate, sometimes rooting at lower nodes; nodes brownish, glabrous; internodes glandular or eglandular below nodes, glabrous. Leaf sheaths often with tufts of hairs at mouth. Ligule ciliate or membranous. Leaf blades linear to lanceolate, apex acute to acuminate, base almost rounded to subcordate, margins eglandular or with raised glands, serrulate, surfaces hairy or glabrous. Inflorescence panicle, open to spiciform or contracted; primary branches altenate to opposite or subwhorled, glandular or eglandular, axils hairy or glabrous; peduncle glandular or nonglandular, glabrous. Spikelets 1-18 $(-23) \times 0.6-9 \mathrm{~mm}$, up to 80 -flowered, ovate, oblong, linear to lanceolate, laterally compressed, straight or slightly curved, greenish, greyish, yellowish, purplish to greenish black. Glumes linear to lanceolate or ovate, apex acute or acuminate, margins entire or ciliate, 1-nerved or nerveless, 1-keeled, or without keel; florets disarticulating from below upwards or from above downwards; rachilla zigzag or nearly straight, slender or stout. Lemmas ovate, lanceolate, oblong or elliptic, apex acute or acuminate or obtuse, margins entire or ciliate, usually 3-nerved, unawned, glandular or eglandular, 1-keeled; keel scaberulous or glabrous. Paleas caducous or persistent, apex acute, acuminate or obtuse, entire or sliced to 3-lobes, flap margins entire or ciliate, 2-nerved, nerves reach at apex, or up to $3 / 4$ length of palea, 2-keeled; keels scaberulous, ciliate or eciliate. Lodicules 2 , obovate to cuneate, apex truncate 
or dentate, white- or cream-coloured. Stamens 3 or 2; anthers $0.2-10 \mathrm{~mm}$ long, purplish or yellowish. Ovary ovoid, obovoid or ellipsoid, light green, white- or cream-coloured; styles free to the bases, white; stigmas plumose, whiteor cream-coloured or purplish. Caryopses ovoid, obovoid, ellipsoid, oblong, globular, laterally or dorsally compressed, angled with or without groove, truncate, obtuse or acute at ends, brownish to yellowish or deep brown in colour.

Key to the species

1a. Spikelets disintegrating at maturity; caryopses variously shaped

1b. Spikelets not disintegrating at maturity; caryopses spindle-shaped

...35. E. tef

2a. Glumes, lemmas and paleas disarticulating as entire unit of spikelet from pedicels ...34. E. superba

2b. Glumes, lemmas and paleas disarticulating one after another from rachilla ...3

3a. Spikelets disarticulating from above downward

3b. Spikelets disarticulating from below upward

4a. Lemmas and paleas ciliate on margins

4b. Lemmas and paleas eciliate on margins

5a. Panicles much compact, cylindric; stamens 2

...7. E. ciliata

5b. Panicles less compact, not cylindric; stamens 3

6a. Spikelets less than $4.5 \times 2 \mathrm{~mm}$; lemmas up to $1.7 \mathrm{~mm}$ long, chartaceous; palea keels not winged; keel cilia long, stiff, thick and bulbous-based; caryopses less than $0.5 \mathrm{~mm}$ long

6b. Spikelets up to $8 \times 3 \mathrm{~mm}$; lemmas up to $3 \mathrm{~mm}$ long, subcoriaceous; palea keels narrowly winged; keel cilia short, thin and not bulbous-based; caryopses up to $0.6 \mathrm{~mm}$ long

7a. Ligules purely membranous; paleas incise into 3 lobes at apex ...17. E. japonica

7b. Ligules a fringe of cilia; paleas acute to obtuse at apex, not lobed

8a. Panicles much spreading and broom like with long and capillary branches; pedicels capillary and flexuous; lemmas truncate at apex; palea keels scaberulous

8b. Panicles open or spiciform with more or less stiff branches; lemmas acute to obtuse at apex; palea keels ciliate

9a. Peduncles, panicle branches and pedicels eglandular or obscurely glandular; palea keels tubercle-based ciliate; cilia subequal or longer than the width of palea

9b. Peduncles, panicle branches and pedicels prominently glandular; palea keels ciliate but cilia not tubercle-based; cilia subequal or shorter than the width of palea

10a. Lemmas dorsally with 2-3 mm long cilia along median nerve towards base; lateral nerves excurrent; anthers 2

10b. Lemmas dorsally scabrid or glabrous on median nerve; lateral nerves not excurrent; anthers 3

11a. Annual; panicles open or narrowly spiciform, viscous

11b. Perennial; panicles compact or spiciform, non-viscous

12a. Plants prominently glandular on culms, leaves, peduncle, panicle branches, pedicles or nerves of glumes and lemmas

12b. Plants eglandular 
13a. Peduncle viscous below panicle

13b. Peduncle non-viscous

14a. Primary panicle branches capillary or filiform; spikelets less than $1 \mathrm{~mm}$ wide

14b. Primary panicle branches more or less stiff; spikelets more than $1 \mathrm{~mm}$ wide

15a. Leaf margins eglandular

15b. Leaf margins conspicuously glandular

16a. Annual; caryopses ovoid to subglobose or ellipsoid, less than $0.7 \mathrm{~mm}$ long

16b. Perennial; caryopses strongly flattened to grooved, more than $0.7 \mathrm{~mm}$ long

17a. Spikelets serrate in appearance; glumes nerveless or nerves obscure; lemma lateral nerves indistinct

17b. Spikelets not serrate in appearance; glumes distinctly 1-nerved; lemma lateral nerves distinct $\ldots 18$

18a. Florets closely arranged on rachilla; rachilla not visible between florets; rachilla internodes less than $1 \mathrm{~mm}$ long

18b. Florets distantly arranged; rachilla clearly visible between florets; rachilla internodes more than $1 \mathrm{~mm}$ long

19a. Spikelets less than $1.3 \mathrm{~mm}$ wide; glumes unequal; caryopses truncate at ends

19b. Spikelets more than $1.3 \mathrm{~mm}$ wide; glumes subequal; caryopses not truncate at ends

20a. Leaf blades up to $8 \mathrm{~mm}$ wide; panicles broadly ovate to obong, with spikelets clustered on branches; lemmas up to $4 \mathrm{~mm}$ long

20b. Leaf blades less than $4 \mathrm{~mm}$ wide; panicles narrowly ovate to oblong with spikelets scatterely arranged on branches; lemmas less than $2.5 \mathrm{~mm}$ long

21a. Spikelets up to 55-flowered, linear, more or less curved, less than $2 \mathrm{~mm}$ wide; caryopses ovoid to ellipsoid, ventrally flattened to slightly grooved

21b. Spikelets up to 45-flowered, ovate, oblong or elliptic, not curved, more than $2 \mathrm{~mm}$ wide; caryopses ellipsoid to subglobose or oblong, not flattened

22a. Paleas acuminate at apex; nerves of palea not reach up to apex; palea keels ciliate

22b. Paleas acute to obtuse at apex; nerves of palea reach up to apex; palea keels scaberulous or ciliolate

23a. Paleas not persistent (sub-persistent in E. gangetica) on rachilla nodes

23b. Paleas persistent on rachilla nodes

24a. Rachilla slender and clearly visible between florets; spikelets less than $1.5 \mathrm{~mm}$ wide; lemmas less than $1 \mathrm{~mm}$ long

24b. Rachilla more or less stiff and not visible between florets; spikelets more than $1.5 \mathrm{~mm}$ wide; lemmas more than $1 \mathrm{~mm}$ long

25a. Paleas narrowly winged; stamens 2; anthers less than $0.5 \mathrm{~mm}$ long

25b. Paleas not winged; stamens 3; anthers more than $0.5 \mathrm{~mm}$ long

26a. Lemma gibbous, apiculate at apex

26b. Lemmas not gibbous, acute or acuminate at apex 
27a. Spikelets dull green to grey; caryopses ellipsoid to oblong

27b. Spikelets pinkish to chocolate brown; caryopses obovoid

28a. Leaves filiform, drooping, apex attenuate into a point

28b. Leaves not filiform, apex not attenuate into a point

29a. Panicles subsecund

29b. Panicles not subsecund

30a. Leaf sheaths and lamina hirsute and tubercle-based-hairy, leaving sunken pits after falling; pedicels barbellate; glumes and lemmas boat-shaped

30b. Leaf sheaths and lamina hairy or glabrous, hairs not hirsute or tubercle-based; pedicels scabrous or nearly glabrous; glumes and lemmas linear to lanceolate, elliptic, ovate or oblong

31a. Spikelets sharply acute to acuminate in outline; lemmas subcoriaceous; palea keels ciliolate

31b. Spikelets not as above; lemmas chartaceous to membranous; palea keels scabrous to scaberulous

32a. Spikelets scatterely arranged on panicle branches ...3. E. brownii

32b. Spikelets fascicled, more or less clustered on panicle branches $\ldots 33$

33a. Leaves flat; spikelets up to 60 -flowered; lemmas up to $2.5 \mathrm{~mm}$ long; paleas up to $1.5 \mathrm{~mm}$ long

33b. Leaves filiform; spikelets up to 20 -flowered; lemmas less than $1.8 \mathrm{~mm}$ long; paleas less than $1.25 \mathrm{~mm}$ long

34a. Spikelets 10-70 or more-flowered, longer and curved on capillary and flexuous pedicels; lemmas broady ovate

...38. E. tremula

34b. Spikelets up to 18-flowered, shorter, straight; lemmas ovate to lanceolate or oblong to elliptic

35a. Lemmas more than $2 \mathrm{~mm}$ long

35b. Lemmas less than $2 \mathrm{~mm}$ long

36a. Spikelets blackish or dark grey; lemmas acuminate at apex ...23. E. nigra

36b. Spikelets greenish to yellowish; lemmas acute or obtuse at apex

39. E. trichodes

37a. Caryopses laterally flattened ...38

37b. Caryopses dorsally or ventrally flattened or groved ...39

38a. Panicles open or narrowly contracted, lowermost branches alternate; spikelets more than $2 \mathrm{~mm}$ wide; caryopses $0.5-0.8 \mathrm{~mm}$ long

38b. Panicles lax to effuse, lowermost branches whorled; spikelets less than $1 \mathrm{~mm}$ wide; caryopses $0.4-0.5 \mathrm{~mm}$ long

39a. Panicles more or less contracted, branches appressed; lemmas up to $1.3 \mathrm{~mm}$ long; caryopses globose to ellipsoid

39b. Panicles open to effuse, branches spreading; lemmas up to $1.7 \mathrm{~mm}$ long; caryopses ovoid to ellipsoid or oblong

40a. Panicle branches alternate or lowermost sub-whorled; spikelets dark green to greyish

40b. Panicle branches strictly alternate; spikelets greenish to purplish 
41a. Spikelets up to $12(-14) \mathrm{mm}$ long; anthers $0.6-0.9 \mathrm{~mm}$ long ...18. E. lehmanniana

41b. Spikelets up to $6 \mathrm{~mm}$ ling; anthers less than $0.5 \mathrm{~mm}$ long ...19. E. macilenta

42a. Pedicels capillary, up to $2.4 \mathrm{~cm}$ long; spikelets $1-2 \mathrm{~mm}$ wide; palea keels scaberulous throughout; palea flaps almost equal in width to body of palea; caryopses dorsally flattened

42b. Pedicels more or less stiff, up to $1 \mathrm{~cm}$ long; spikelets $c$. $1 \mathrm{~mm}$ wide; palea keels scaberulus above middle; palea flaps narrower than body of palea; caryopses dorsally flattened

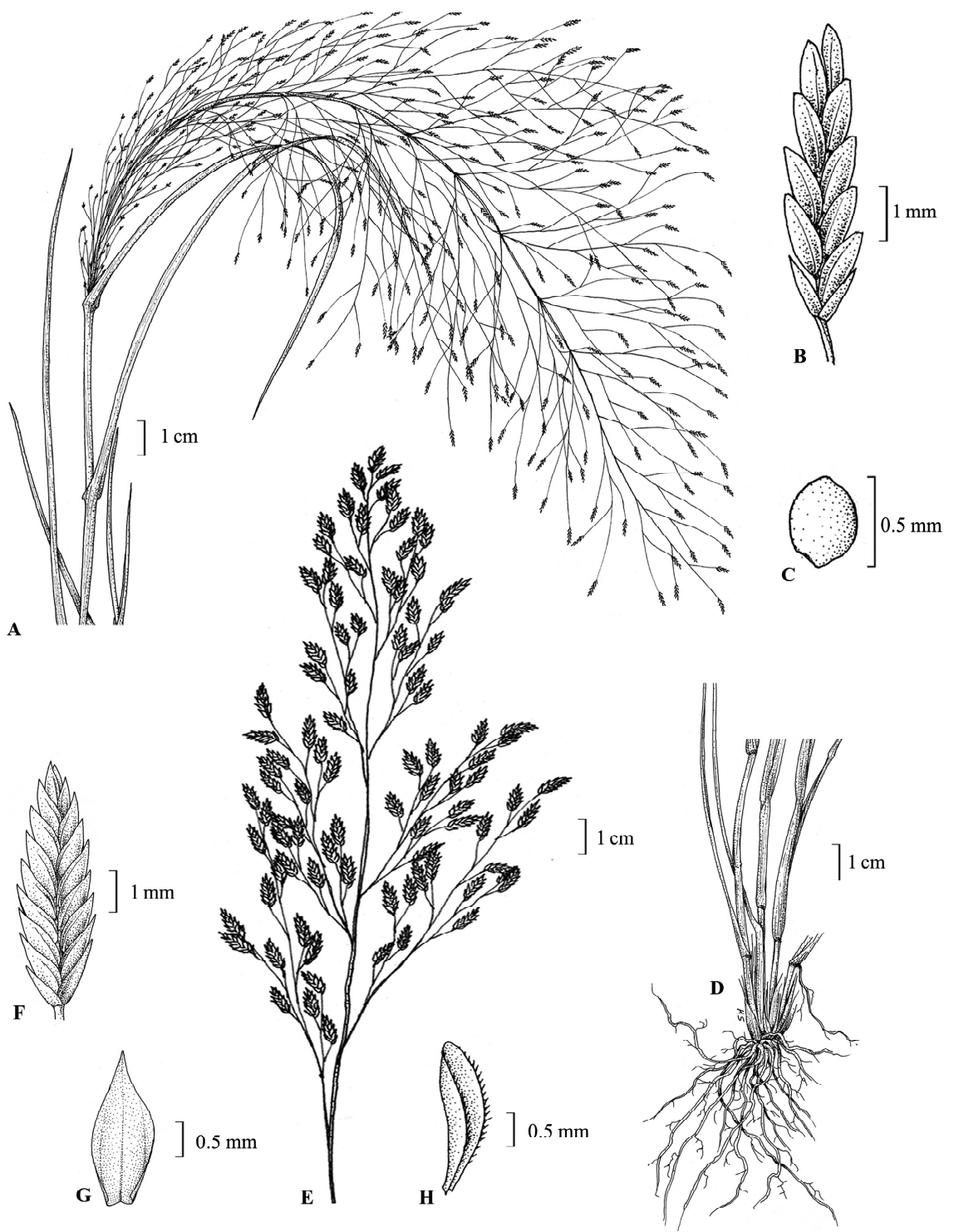

Fig. 1. Eragrostis aspera (Jacq.) Nees (A-C). A. Inflorescence \& culm; B. Spikelet; C. Caryopsis. E. atrovirens (Desf.) Trin. ex Steud. (D-H). D. Culm; E. Inflorescence; F. Spikelet; G. Lemma-ventral view; H. Palea-lateral view. 
1. Eragrostis aspera (Jacq.) Nees, Fl. Afr. Austral. Ill.: 408. 1841

Poa aspera Jacq., Hort. Bot. Vindob. 3: 32, t. 56. 1777. Type: Cultivated in EUROPE with seed from INDIA, s.d., s.coll., s.n. (W [W0000343 image!]).

(Fig. 1 \& Plate 1)

Annual. Culms $40-110 \mathrm{~cm}$ high, erect to suberect; nodes brownish; internodes $c .10 \mathrm{~cm}$ long. Leaf sheaths c. $6 \mathrm{~cm}$ long, with tubercle-based cilia and bearded mouth. Ligule a fringe of cilia. Leaf blades 7-48 $\times 0.4-$ $0.8 \mathrm{~cm}$, flat, upper surface scabrid. Panicles $20-70 \times 7-$ $20 \mathrm{~cm}$, open, thyrsiform, broadly oblong; primary branches alternate to subwhorled, capillary; axils ciliate; pedicels up to $7 \mathrm{~mm}$ long, capillary. Spikelets $4-10 \times$ 1-1.5 mm, 4-22-flowered, linear to oblong, green or green with pinkish to purplish tinge; rachilla zigzag; florets more or less closely arranged on rachilla, disarticulating from above downwards. Glumes ovate to lanceolate, chartaceous, 1-nerved, 1-keeled, scabrous along keel, apex acute; lower glume 1-1.2 ×c. $0.3 \mathrm{~mm}$; upper glume $1.1-1.3 \times$ c. $0.3 \mathrm{~mm}$. Lemmas $1.2-1.7 \times$ 0.4-0.5 mm, ovate to elliptic, chartaceous, 3-nerved, lateral nerves prominent, almost straight, at times slightly excurrent, 1-keeled, scabrid along keel, apex obliquely truncate, obtuse when spread. Paleas $1-1.3 \times$ c. $0.3 \mathrm{~mm}$, caducous, elliptic to lanceolate, 2-nerved, 2keeled, keels scaberulous above middle, apex acute to obtuse; palea flaps narrower than its body. Anthers 3, $c$. $0.2 \mathrm{~mm}$ long, purplish. Caryopses $0.4-0.5 \times 0.3-0.35$ $\mathrm{mm}$, ovoid to subglobose, deep brown.

Flowering and fruiting: July-December; stray flowering almost throughout the year.

Distribution: INDIA: Andhra Pradesh, Bihar, Karnataka Maharashtra, Odisha, Rajasthan and Tamil Nadu; TROPICAL AFRICA, MALESIA, MASCARENE ISLANDS.

Habitat: Grows along road sides of hill slopes, up to 350 m elevation; also in sandy loam soils, moist and shady habitats, and weed in cultivated land.

Chromosome no.: 2n = 20 (Moffett \& Hurcombe, 1949).

Notes: The attractive spreading, broom-like panicle makes this species very distinct from other species of Eragrostis in India.

Specimens examined: INDIA. Andhra Pradesh: West Godavari District: Peddavegi-Ellore, 09.10.1930, V. Narayanaswamy 4516 (MH). Karnataka: Bellari District: Kudatini, 17.11.1979, B.R. Ramesh \& S.R. Ramesh 10513 (CAL); Chamarajanagar District:
Kollegal, Anaikkaraimalai-Lokkanahalli, $\pm 990 \mathrm{~m}$, 15.01.1930, T.R. Naganathan 19480 (MH); Haveri District: Haveri, 01.03.1890, W.A. Talbot 2275 (CAL). Maharashtra: Bombay State (Solapur District), Pandharpur, 05.12.1956, S.C. Agarwal 1001 (CAL). Tamil Nadu: Coimbatore District: Anaikkatti, \pm 609 m, 22.01.1931, S.R. Raju \& Naganathan 4816 (MH); Coimbatore, 17.12.1900, C.A. Barber 2566 (MH); Dinhalli, 13.11.06, C.E.C. Fischer s.n. (FRC); Grattimalai-Slope, $870 \mathrm{~m}, 25.02 .1963$, C.P. Sreemadhavan $466(\mathrm{MH})$; On the way to Kunjur, \pm 853 m, 27.01.1931, S.R. Raju \& Naganathan 4994 (MH); Kuridimalai, 27.11.1956, K. Subramanyam 1468 (CAL); Madeswaram Malai, $\pm 1219 \mathrm{~m}, 10.02 .1930, V$. Narayanaswami 19767 (MH); Manguli, $880 \mathrm{~m}$, 16.12.1970, M.V. Viswanathan $850(\mathrm{MH})$; Pungangeri R.F. - Siruvani, \pm 502 m, 26 Jan. 1932, M. Ramavelu 97 (MH); Velliangiri Hills, 15.01.2012, C.P. Vivek 126148 (MH); Cuddalore District: South Arcot, 06.02.1983, K. Ramamurthy 77344 (CAL); Dindigul District: Sirumalai - on the way, $900 \mathrm{~m}, 26.02 .1978, M$. Chandrabose 54300 ( $\mathrm{MH})$; Kallakurichi District: Gomiki-Vellimalai section, 200 m, 06.02.1983, K. Ramamurthy 77344 (MH); Kanyakumari District: Thekkumalai, 662 m, 19.11.1956, K.M. Sebastine 1386 (CAL \& MH); 666 m, 06.12.1956, K.M. Sebastine 1638 (CAL \& MH); Nilgiris District: Mannar, 06.11.1900, C.A. Barber 2662 (MH); Tirunelveli District: Thirunelveli, 26.11.1969, B.V. Shetty 32916 (CAL); Kodamady Estate - Thirukurungudi, 220 m, 26.11.1969, B. V. Shetty $32916(\mathrm{MH})$.

2. Eragrostis atrovirens (Desf.) Trin. ex Steud., Nomencl. Bot., ed. 2, 1: 562. 1840.

Poa atrovirens Desf., Fl. Atlant. 1: 73, t. 14. 1798. Type: ALGERIA: Barbaria, La Calle, s.d., Desfontaines 160 (holo F [FI012554 image!], picture K, iso BAA-1006 \& P).

(Fig. 1 \& Plate 1)

Perennial. Culms 30-135 cm high, erect to decumbent, geniculate, at times glaucous; nodes brownish; internodes 3-20 cm long. Leaf sheaths 4-7 cm long, with bearded or glabrous mouth. Ligule a ciliate rim, with $0.2-0.5 \mathrm{~mm}$ long narrow membrane at base. Leaf blades $8-22 \times 0.2-0.4 \mathrm{~cm}$, linear to lanceolate, upper surface scabrid. Panicles $8-35 \times 2-8 \mathrm{~cm}$, open, effuse; primary branches up to $10 \mathrm{~cm}$ long, alternate; axils glabrous; pedicels $0.5-5 \mathrm{~mm}$ long. Spikelets $4-10 \times$ 1.5-2.5 mm, 6-20-flowered, ovate to lanceolate, dull green or grey with or without purplish tinge, at times glaucous; rachilla zigzag; florets closely arranged on rachilla, disarticulating from below upwards. Glumes 
linear to lanceolate, chartaceous, 1-nerved, 1-keeled, scabrid along keel towards apex, apex acute; lower glume 1.5-1.8 × 0.3-0.4 mm; upper glume 1.9-2.1 $\times$ $0.4-0.5 \mathrm{~mm}$. Lemmas $1.8-2.2 \times 0.8-1 \mathrm{~mm}$, ovate to lanceolate, chartaceous, 3-nerved, lateral nerves straight, 1-keeled, scabrid along keel towards apex, apex acute to subacuminate. Paleas $1.8-2 \times 0.3-0.4 \mathrm{~mm}$, caducous, elliptic to lanceolate, 2-nerved, 2-keeled, scaberulous along keels, apex acute; palea flaps narrower than its body. Anthers 3, 0.6-0.8 mm long. Caryopses $0.6-0.7 \times 0.3-0.4 \mathrm{~mm}$, ellipsoid to oblong, deep brown.

Flowering and fruiting: Almost throughout the year.

Distribution: INDIA: Almost throughout; AFRICA, AMERICA, AUSTRALIA, JAPAN, MALESIA, MARIANA ISLANDS, MASCARENE ISLANDS, MYANMAR, SRI LANKA and TAIWAN.

Habitat: Grows along drains in sandy soils, along the banks of backwaters, rivers, streams, canals; also in wetlands, in hilly areas, weed in waste places and plantations.

Chromosome nos.: $2 \mathrm{n}=40$ (de Wet, 1960), $2 \mathrm{n}=60$ (Larsen, 1963; Christopher \& Abraham, 1974; Gould \& Soderstrom, 1974), 2n = 20 (Kammacher \& al., 1973).

Notes: There are two forms of E. atroviresns in India, one with tufted and glaucous culms and the other with non-glaucous and comparatively slender culms. The panicle and spikelets of the second form resemble those of E. gangetica. However, there are clear differencences including caducous paleas and oblong caryopses in E. atrovirens versus the sub-persistent paleas and ovoid to ellipsoid caryopses in E. gangetica (Vivek \& al., 2013e).

Specimens examined: INDIA: Andhra Pradesh: Ananthapuramu District: Reddy Palli Farm, 340 m, 15.08.1981, N. Yesoda 281 (MH); Chittoor District: Horslykonda, 05.05.1918, s.coll. 15476 (MH); Kambakam Hills, 06.05.1913, s.coll. 8954 (MH); Kuppam, 26.08.1918, s.coll. 15539 (MH); Seethalam Riverbed, Thandipandal, 225 m, 25.09.1974, M. Chandrabose 45101 (MH); Kadapa District: Balapalle, 320 m, 19.07.1762, J.L. Ellis 14271 (CAL); Kurnool District: Diguvametta, 07.08.1972, J.L. Ellis 42180 (MH); Gundlakamma, Nallamalais, 650 m, 01.04.1965, J.L. Ellis 23838 (MH); Sunnipanta Vagu, $450 \mathrm{~m}$, 21.10.1964, J.L. Ellis 22130 (MH); Visakhapatnam District: Ananthagiri, $1125 \mathrm{~m}, 18.10 .1964, G . V$. Subbarao 21759 (MH); Chalikona summit waterfall,
304 m, 29.01.1934, K.C. Jacob 453 (MH); Khotnighada area, 910 m, 12.05.1964, G.V. Subbarao 19505 (MH); Matryagundam, 1000 m, 03.06.1968, G.V. Subbarao $30067(\mathrm{MH})$; Bihar: Champaran District: 07.04.1963, K. Thothathri 9937 (CAL); Manguraha Forest, 14.04.1963, K. Thothathri 10028, 10091 (CAL); Bilaspur District: Bilaspur, 29.10.1970, G. Panigrahi 13057 (CAL); North Bilaspur, 22 Dec. 1969, C.M. Arora 7126 (CAL); Dantewada District: Bailadila, 13.02.1963, G. Panigrahi 1689 (CAL). Chhattisgarh: Bastar District: Keskal, 567 m, 17.11.1958, K. Subramanyam 7121 (CAL \& MH). Himachal Pradesh: Kangra District: s.d., Stoliczka s.n. (CAL). Jharkhand: Ranchi District: Karo river, 17.02.1981, K.C. Malik 9721 (CAL); Sahibganj District: Rajmahal, Oct.1894, Mokim 1510 (CAL). Karnataka: Chamarajanagara District: Doda Halla, Kollegal, \pm 792 m, 05.07.1930, V. Narayanswami 3610 (MH); Coorg (Kodagu) District: Fraserpet, 1902, C.A. Barber 4419 (MH); Hassanur, \pm 914 m, 21.06.1929, K.C. Jacob 18552 (MH); Hassan District: Byra, 11.11.1978, C.J. Saldanha \& P. Prakash 3793 (CAL); Mandya District: Sivasamudra, 28.10.1978, S.R. Ramesh \& S.B. Manohar 3966 (CAL); Mysore District: Bandipur Forest, 28.01.1965, B.D. Naithani 23202 (MH); Uttara Kannada District: Pilikode, 10.11.1917, s.coll. 15331, 15337 (MH). Kerala: Idukki District: Edapalayam, 850 m, 25.09.1972, B.D. Sharma 41677 (MH); Mangaladevi lake, 1100 m, 16.02.1981, N.C. Nair 70231 (MH); Mullakkudy, 850 m, 1973, B.D. Sharma 43880 (MH); Munnar, 30.05.1944, K.C. Jacob 20484 (MH); Munnar, 18.12.2008, K.K. Suresh 4480 (Herbarium of Malabar Botanical Garden and Institute for Plant Sciences, Kozhikode, Kerala); Thenkachi, 24.09.1972, B.D. Sharma 40891 (MH); Thekkady, 850 m, 15.03.1973, B.D. Sharma 43880 (MH); Kannur District: Aralam, 250 m, 26.02.1979, V.S. Ramachandran 61935 (CAL \& MH); Aralam Farm, 16.08.1979, 250 m, V.S. Ramachandran 63966 (MH); Chandanathode, $850 \mathrm{~m}$, 15.06.1979, V.S. Ramachandran 62629 (CAL \& MH); Thirunelli, 725 m, 04.03.1979, V.S. Ramachandran 62069 (MH); Tolpetty Forest, 725 m, 08.02.1978, V.S. Ramachandran 52393 (MH); Kasaragod District: Peelikoode, 06.02.2007, K.K. Suresh 3155 (Herbarium of Malabar Botanical Garden and Institute for Plant Sciences, Kozhikode, Kerala); Kollam District: Thenmala, 300 m, 18.05. 1978, M. Mohanan 54857 (CAL \& MH); Thenmalai, $450 \mathrm{~m}, 19.05 .1978$, C.N. Mohanan 55593 (CAL \& MH); Kottayam District: Changanassery, Kurisumala, 1000 m, V.T. Antony 934 (MH); Mudurapalai river side, 525 m, 13.06.1963, K.M. Sebastine 16441 \& 20839 (MH); Kozhikode District: 
Nilanjara, Chedaleth, 900 m, 14.08.1964, J.L. Ellis 19992 (MH); Palakkad District: Chindakk Forest, 12.01.1980, N.C. Nair 65419 (MH); Pulikal Forest, 20.07.1964, K.M. Sebastine 20839 (MH); Vetriluichola, Pulikal, 1200 m, 20.07.1964, K.M. Sebastine 20839 (MH); Thiruvananthapuram District: Agasthyamala, western slope, 1800 m, 06.10.1973, J. Joseph 44638 (MH); Kottur, 04.05.1979, P. Farooqui \& V.K. Nallasamy 781 (FRC); Klamala R.F., 200 m, 14.04.1973, J. Joseph 44163 (MH); Neyyar, 175 m, 26.05.1979, M. Mohanan 63288 (CAL \& MH); Neyyar dam site, 15.01.2009, K.K. Suresh 4607 (Herbarium of Malabar Botanical Garden and Institute for Plant Sciences, Kozhikode, Kerala); Thrissur District: Chalakkudi river side, Kodasseri, 300 m, 22.03.1980, K. Ramamurthy 66287 (CAL \& MH); Kandasan Kadavu pond, 10 m, 07.09.1976, K. Ramamurthy 48403 (MH); Thunakkadavu submergible area, 667 m, 10.03.1965, K.M. Sebastine 22855 (MH); Wayanad District: Karappuzha Dam site, 19.02.2008, K.K. Suresh 4871 (Herbarium of Malabar Botanical Garden and Institute for Plant Sciences, Kozhikode, Kerala); Meppadi, 20.06.1902, C.A. Barber 4217 (MH). Madhya Pradesh: Damoh District: 11.02.1979, B.K. Shukla 29745; Sirghrampur, 17.09.1979, B.K. Shukla 29981 (CAL); Jubbulpur District: 13.03.1962, K.M. Sebastine 13959 (CAL); Kundwara, SW of forest Bungalaw, 472 m, 13.03.1962, K.M. Sebastine 13952 (MH); Kundwara R.F., 475 m, 23.12.1962, K.M. Sebastine 15522 (MH); Mahadeo, 27.07.1964, G. Panigrahi \& V. Singh 4549 (CAL); Mandhla District: Kanta, 08.03.1962, J.K. Maheshwari 4710 (CAL); Rewa District: 13.02.1959, K.M. Sebastine 7736 (CAL); Sidhi District: 17.01.1964, G. Panigrahi 2152 (CAL); Mandla District: Khari R.F., 675 m, 27.11.1961, J. Joseph 13440 $(\mathrm{MH})$; Rewa District: Agri College Rewa, beside the lake, 500 m, 13.02.1959, K.M. Sebastine 7736 (MH); Saugor District: Timorda, near Hirapur, $420 \mathrm{~m}$, 03.03.1960, K. Subramanyam 10175 (MH); Shivpuri District: Naka Kota, 12.08. 1963, D.M. Verma 445 (CAL). Maharashtra: North Kanara District: 26.11.1889, W.A. Talbot 2067 (CAL). Meghalaya: Cherapunji District: 01.06.1911, S.C. Banerjee 159 (CAL); Khasi District: Khasia, s.dat., J.D. Hooker s.n., MH Acc. No. 87758 (MH); Nungpo, Khasia, $\pm 1371 \mathrm{~m}$, 30.10.1872, C.B. Clarke 17686 (CAL); Shillong District: 20.09.1959, S.C. Deka 18381 (CAL); Shillong, 1878, Geo Gallatly 158 (CAL). Odisha: Angul District: 14.12.1902, J.H. Lace 2589 (CAL); Ganjam District: Mahendragiri, $\pm 1158 \mathrm{~m}, 17.08$. 1931, $V$. Narayanaswami 5743 (MH). Rajasthan: Ganganagar District: 17.11.1976, G.P. Roy 3958 (CAL); Padampur,
29.10.1959, G. Panigrahi 20543 (CAL). Sikkim: East Sikkim District: Gangtok, s.d., N.C. Mazumdar 37 (CAL); Rhenock, 12.12.1980, P. Chakraborti 1144 (BSHC). Tamil Nadu: Coimbatore District: Akkamalai, 1625 m, 18.02.1980, M. Chandrabose 65853 (MH); Aliyar submergible area, $350 \mathrm{~m}, 25.11 .1962$, K.M. Sebastine 15377 (MH); Kallar R.F., \pm 1066 m, 01.03.1942, S.R. Raju 20308 (MH); Kobai Halla bank, Hassanur, \pm 914 m, 09.03.1931, K.C. Jacob 170 (MH); Maruthamalai, 653 m, 07.08. 1956, K.M. Sebastine 510 (MH); Muthukolam, Siruvani, 1067 m, 28.05.1957, K. Subramanyam 3062 (MH); Ollear to Thorakadavur way, 10.10.1901, C.A. Barber 3672 \& $4182(\mathrm{MH})$; Siruvani, Adivaram, 667 m, 15.02.1957, K. Subramanyam 2339 (MH); Erode District: Dhinbam to Bannari, road side, 04.10.1987, N. Venkatasubramanyan 869 (FRC); Kanyakumari District: Muthukuzhi Estate, 1400 m, 27.07.1977, A.N. Henry 49408 (MH); Muthukuzhi Vayal, 1400 m, 28.09.1980, A.N. Henry 68845 (MH); PurathimalaiKulasekaran range, $200 \mathrm{~m}, 27.01 .1978$, A.N. Henry $53323(\mathrm{MH})$; Madurai District: Combai, 333 m, 16.09.1961, K.M. Sebastine 12926 (MH); Kumbukere, 400 m, 15.06.1961, K.M. Sebastine 12602 (MH); Kumili, 830 m, 25.06.1959, K. Subramanyam 8209 (MH); Periyar River bank, 925 m, 23.04.1960, B.V. Shetty 10265 (MH); Nilgiris District: s.loc., Aug. 1883, J.S. Gamble 12339 (CAL); Coonoor,.06.1883, J.S. Gamble 12131 (CAL); Coonoor, along railway track, 700 m, 22.10.2011, C.P. Vivek 126154 (MH); Ebanad Stream Bank, 900 m, 29.08. 1970, G. V. Subbarao 36411 (MH); Hulical Droog, 1733 m, 27.08. 1957, K.M. Sebastine 4132 (MH); Karimund, Pykara, 2060 m, 13.07.1970, J.L. Ellis 34660 (MH); Kodagu Road, 05.01.1957, K. Subramanyam 1942 (CAL); Koilmattam, near Ebanad, 1850 m, 25.03.1972, G.V. Subbarao 40478 (MH); Kotagiri,.09.1885, J.S. Gamble 16725 (CAL); Kundha, 16.05.1957, K.M. Sebastine 3278 (CAL); Kunnacombai, 2000 m, 16.05.1957, K.M. Sebastine 3278 (MH); Loz Falls, 1450 m, 12.03.1969, D.B. Deb 31708 (MH); Ootacamund,.01.1902, C.A. Barber 4182 (MH); Ootty, June 1883, J.S. Gamble 11736 (CAL); Pakasura hills, 200 m, 08.12.1957, K.M. Sebastine 4825 (MH); Parkside R.F., $2000 \mathrm{~m}$, 29.03.1958, K.M. Sebastine 5677 (CAL \& MH); Pykara, 1828 m,.05.1889, J.S. Gamble 20514 (MH); Salem District: Hogainakal, 11.02.1927, K.C. Jacob 17994 (MH); Hogainakal Forest, 275 m, 16.07.1964, E. Vajravelu 20658 (MH); Yercaud, 1380 m, 15.02.1969, D.B. Deb 31415 (MH); Tirunelveli District: Ambasamudram, 13.05.1901, C.A. Barber 2745 (MH); 
Courtallam Falls, 333 m, 21.04.1957, K. Subramanyam 2792 (MH); Kalakkadu, 225 m, 12.11.1962, J. Joseph $15262(\mathrm{MH})$; Tiruchirapalli District: Puliyancholai river bank, 233 m, 29.10.1958, K.M. Sebastine 7042 (MH). Telangana: Karimnagar District: Kodimial, 400 m, 18.07.1964, G.V. Subbarao 20132 (MH); Mahadevpur, nearby Neelampalli, 100 m, 22.02.1986, N. Rama Rao \& T. Ravishankar 83712 (MH); Warangal District: Pakhal, 290 m, 25.02.1963, A.N. Henry 15911 (MH); Pakhal, beside the lake, $290 \mathrm{~m}, 30.11 .1960$, K.M. Sebastine $11702(\mathrm{MH})$. Tripura: Agartala District: Tipperah, 20.12.1914, P.M. Debberman 334 (CAL). Uttar Pradesh: Aligarh District: 11.11.1887, J.F. Duthie 6779 (CAL); Mirzapur District: 05.10.1969, G. Panigrahi 12280 (CAL); Satna District: Majhgawan, 23.02.1987, R. Prasad 38456 (CAL); Shahjahanpur District: Indalpur, Gangetic Plain, 11.10.1885, J.F. Duthie 5113 (CAL); Sonbhadra District: Dudhi, 26.10.1964, G. Panigrahi 2073 (CAL); Varanasi District: Kotwa, 15.07.1965, O.P. Misra 9701 (CAL). West Bengal: Birbhum District: Moshmara Forest, 18.12.1969, R.K. Basak 1263 (CAL); Goalpara, Santiniketan, 26.07.1969, R.K. Basak 1025 (CAL); Burdwan District: Eden Canal, 16.10.1894, J.D. Hooker 1206 (CAL); Hooghly District: Goghat, 11.03.1902, J.D. Hooker 35 (CAL); Purulia District: s.dat., K.C. Malik 1039 (CAL).

3. Eragrostis brownii (Kunth) Nees, Cat. Indian Pl.: 105. 1834.

Poa brownii Kunth, Revis. Gramin. 1: 112. 1829; nom. nov. for Poa polymorpha R.Br. Prodr. 180. 1810, non Wibel 1799. Lectotype: AUSTRALIA, Queensland, Port Curtis District, R. Brown 6284 (BM, K images!), designated by Lazarides in Austral. Syst. Bot. 10: 101. 1997.

Uniola spicata Llanos, Fragm. Pl. Filip.: 33. 1851, non L. 1753. Neotype: PHILIPPINES, Luzon, E.D. Merrill Sp. Blancoan 170 (US, image!, iso K image!), designated by Veldkamp in Blumea 47: 169. 2002.

Eragrostis spartinoides Steud., Syn. Pl. Glumac. 1: 265. 1854. Type: PHILIPPINES, Luzon, Laguna, Cuming 668 (holo $\mathrm{P}$, image!, iso $\mathrm{K}$, image!).

Eragrostis santapaui K.G. Bhat \& Nagendran in Reinwardtia 10: 127. 1985. Type: INDIA, Karnataka, Coorg District, Mercara, 18 December 1980, K.G. Bhat 794A (holo CAL!, iso K image!).

(Plate 1)

Perennial. Culms 10-68 cm high, erect or ascending; nodes brownish; internodes $4-14 \mathrm{~cm}$ long. Leaf sheaths 3-6 cm long, glabrous, with bearded mouth. Ligule a rim of cilia with narrow membrane at base. Leaf blades 2-20 $\times 0.1-0.4 \mathrm{~cm}$, linear to lanceolate, upper surface sparsely ciliate, glabrous when mature. Panicles 10-28 $\times 4-11 \mathrm{~cm}$, open; primary branches $1-6 \mathrm{~cm}$ long, alternate, starts dividing almost from near base; axils ciliate. Spikelets 4-20 × 1.5-2.8 mm, 5-42-flowered, oblong to lanceolate or ovate, sharply acute, light green to grey; rachilla persistent, zigzag; florets firmly arranged on rachilla, disarticulating from below upwards. Glumes elliptic to lanceolate, membranous to chartaceous, 1-nerved, 1-keeled, glabrous or scabrid along keel, apex acute; lower glume $0.8-1.5 \times 0.25-0.7$ $\mathrm{mm}$; upper glume $1-1.9 \times 0.4-0.8 \mathrm{~mm}$. Lemmas $1.4-$ $2.8 \times 0.6-1.5 \mathrm{~mm}$, lanceolate to elliptic or narrowly ovate, chartaceous or membranous, 3-nerved, lateral nerves distinct, 1-keeled, scabrous or glabrous on keel, apex acute to subacuminate. Paleas 1.2-1.8 $\times 0.6-0.9$ $\mathrm{mm}$, persistent, oblanceolate or obovate, slightly curved, 2-nerved, 2-keeled, ciliolate along keels; palea flaps narrower than its body. Anthers 3, 0.3-0.6 mm long, purplish to brownish. Caryopses $0.3-0.8 \times 0.3-$ $0.5 \mathrm{~mm}$, subglobose to oblong, brownish.

Flowering and fruiting: August-December.

Distribution: INDIA: Karnataka; tropical and temperate regions of the world.

Habitat: The species occurs in moist open places, on roadsides and forest margins.

Specimens examined: INDIA. Karnataka: Coorg (Kodagu) District: Mercara, 18.12.1980, K. Gopalakrishna Bhat 794 (CAL); Shimoga District: Nearby Tirthahalli, 12.12.1978, S.R. Ramesh \& P. Prakash 5281 (CAL). SRI LANKA, without precise locality, 1882, C.P. 176 (MH).

4. Eragrostis burmanica Bor in Kew Bull. 6(2): 166. 1951. Type: MYANMAR, Prome District, at the edge of paddy uelds, 20.07.1948, U Thein Lwin 582 (holo K [K000245112 image!]).

(Fig. 2 \& Plate 4)

Perennial. Culms $35-60 \mathrm{~cm}$ high, erect or ascending, geniculate; nodes brownish, glabrous. Leaf sheaths sparsely ciliate or glabrous, mouth bearded. Ligule a ciliate rim with narrow membrane at base. Leaf blades 6-15 $\times 0.4-0.6 \mathrm{~cm}$, flat or convolute, upper surface scabrous to sparsely ciliate. Panicles c. $35 \times 15 \mathrm{~cm}$, open, lax or effuse; branches up to $15 \mathrm{~cm}$ long, alternate; axils glabrous; pedicels capillary, up to $2.4 \mathrm{~cm}$ long. Spikelets 2-6 $\times 1-2 \mathrm{~mm}, 5-16$-flowered, ovate to oblong or elliptic, purplish; rachilla persistent, slender, zigzag; florets closely arranged on rachilla, disarticulating from below upwards. Glumes elliptic to lanceolate, 
chartaceous, 1-nerved, 1-keeled, scaberulous along keel, apex acute; lower glume $c .1 \mathrm{~mm}$ long; upper glume $c$. $1.25 \mathrm{~mm}$ long. Lemmas $1.1-1.7 \times$ c. $0.8 \mathrm{~mm}$, ovate to elliptic, chartaceous, 3-nerved, 1-keeled, scabrous along keel, apex acute. Paleas 1-1.2 mm long, persistent, oblanceolate to elliptic, 2-nerved, 2-keeled, scaberulous along keels, apex acute; palea flaps narrower than its body. Anthers 3, c. $0.4 \mathrm{~mm}$ long, purplish. Caryopses $0.6-0.75 \times c .0 .3 \mathrm{~mm}$, ellipsoid, slightly flattened dorsally, reddish brown.

Flowering and fruiting: September.

Distribution: INDIA: Manipur (reported here); MYANMAR, THAILAND (Chaisongkrama \&al., 2013).

Habitat: Grows along the margins of paddy fields.

Chromosome nos.: 2n=40 (Larsen, 1963).

Notes: Eragrostis burmanica was described by Bor in 1951 from Burma (Myanmar). According to him it is not common but is found along the margins of paddy fields in the Prome district. In CAL there are 2 sheets of a single collection of Eragrostis made by Bor in 1937 (N.L. Bor 15438) from Imphal District of Manipur, a neighbouring state of the country Myanmar, which were identified and labelled by him only up to the generic level. Someone else determined this as $E$. tremula Hochst. ex Steud. Furthermore, an unknown worker has given the following comment on the sheet "this perennial coming close to E. burmanica, a Burma species.....". On further study of the specimens with the help of the protologue and type images, the specimens were identified as E. burmanica. Bor described E. burmanica based only on his collection of it from Myanmar, though he had collected the same species from India 13 years earlier but could not recognize it at that time, which led him to wrongly mention in the protologue that E. burmanica as an endemic to Myanmar.

Sreedevi \& Binoj Kumar (2001) claimed the first report of E. burmanica in India from Kerala state based on the specimen collected from Thottappally in Alappuzha District. However, there is no mention of the specimen of their claim or presisce locality of the collection or the herbarium wherein the specimen is housed. Besides, it is evident from the description and illustration provided in the publication that the identity of the specimen involved was wrongly confirmed as $E$. burmanica which in fact belonged to a different species of Eragrostis. Leaves of the species described by them are filiform, panicle is narrow, spikelets are curved, greyish green, and more than 20-flowered, which are not the characters of E. burmanica. While the leaves are flat, spikelets are straight, purplish green and less than 16-flowered in E. burmanica. Therefore, the species record in Kerala state (Sreedevi \& Binoj Kumar, 2001 ) is not considered in the present paper.

Specimens examined: INDIA. Manipur: Imphal District: 02.09.1937, N.L. Bor 15438 (CAL). MYANMAR, Prome District, at the edge of paddy ûelds, 20.07.1948, U. Thein Lwin 582 (K).

5. Eragrostis cilianensis (All.) Vignolo ex Janch., Mitt. Naturwiss. Vereins Univ. Wein 5(9): 110. 1907.

Poa cilianensis All., Fl. Pedem. 2: 246, t. 91, f. 2.1785. Lectotype: ITALY, In agrio patrio Ciliana, Cl. Bellardi s.n. (TO-8242), designated by F. Vignolo Lutati in Malpigia 18: 380. 1904.

Briza eragrostis L., Sp. Pl.: 70. 1753. Eragrostis major Host, Icon. Descr. Gram. Austriac. 4: 14, t. 24. 1809, nom. superfl. Lectotype: Herb. Burser I: 10 (UPS), designated by Clayton in Polhill, Fl. Trop. E. Africa, Gram. 2: 232. 1974.

(Fig. 2 \& Plate 1)

Annual. Culms 20-140 cm high, erect or decumbent, with or without a ring or cluster of glands close below nodes; nodes brownish. Leaf sheaths with pitted or warty glands especially on midnerve and margins. Leaf blades $3-19 \times 0.3-0.8 \mathrm{~cm}$, usually flat, mouth often bearded, upper and lower surfaces glabrous. Ligule $0.5-1.5 \mathrm{~mm}$ long, a ciliate rim with narrow membrane at base. Panicles $10-28 \times 3-15 \mathrm{~cm}$, open or dense, ovate to oblong; primary branches $5-8 \mathrm{~cm}$ long, usually with crateriform, pitted or warty glands; axils ciliate; pedicels 1-2 mm long, punctately glandular. Spikelets 5-20 $\times$ 2-4 mm, 4-35-flowered, ovate to oblong, greenish to grey or yellowish; rachilla zigzag with internodes $c$. 0.5 $\mathrm{mm}$ long; florets closely arranged on rachilla, disarticulating from below upwards. Glumes ovate to lanceolate, chartaceous, 1-nerved, 1-keeled, scabrid with 2-4 pitted glands along keel, apex acuminate; lower glume $1.5-2.2 \times 0.4-0.6 \mathrm{~mm}$; upper glume $1.7-2.2 \times$ $0.6-0.8 \mathrm{~mm}$. Lemmas $1.8-2.2 \times 0.8-1 \mathrm{~mm}$, broadly ovate to elliptic, boat shaped, almost orbicular when spread, chartaceous, 3-nerved, 1-keeled, with or without minute glands on keel, apex acute to subacute or obtuse. Paleas $1.7-2 \times 0.5-0.7 \mathrm{~mm}$, persistent, elliptic to oblanceolate, curved, 2-nerved, 2-keeled, ciliolate along keels above middle, apex acute; palea flaps narrower than its body. Anthers 3, c. $0.3 \mathrm{~mm}$ long yellowish to cream coloured. Caryopses c. $0.4 \times 0.4 \mathrm{~mm}$, globose to orbicular, reddish brown. 
Flowering and fruiting: January-September; stray flowering almost throughout the year.

Distribution: INDIA: Almost throughout; tropical parts of the world.

Habitat: Grows in moist open places, in cultivated fields, forest margins, hillslopes, riverbeds, etc., usually grows up to $1000 \mathrm{~m}$.

Vernacular name: Bettada akabu hullu (Kannada).

Chromosome nos.: 2n = 20 (Moffett \& Hurcombe, 1949; Malik \& Tripathi, 1970; Dujardin, 1979), 2n = 70 (Mehra \& al., 1968).

Notes: Genereal appearance, presence of crateriform glands on plant parts viz. leaf margins, peduncle, panicle branches, pedicels and lemma keels place this species closer to E. minor. However, E. cilianensis is more glandular having larger panicles and broader spikelets.

Specimens examined: INDIA. Himachal Pradesh: Shimla District: Rohru, 1800 m, 31.07.1965, N.C. Nair 36223 (BSD). Karnataka: Chamarajanagara District: Anakasai Malai-Lokkanahalli, \pm 975 m, 15.01.1930, V. Narayanaswami 19479 (MH); Belgaum District: August, W.M. Munro 847 (CAL); Bellary District: Kottur, 12.10.1919, s.coll. 15961 (MH); Hagari, 20.09.1916, s.coll. 15930 ( $\mathrm{MH})$; Ramandurg, 09.10.1919, s.coll. 15930 (MH); Mysore District: Felaigi, 05.10.1903, C.A. Barber 6137 (MH); Mysore, s.dat., G. Thomson s.n. (CAL); St. Philomena's College, $725 \mathrm{~m}$, 10.03.1964, K.M. Sebastine 18715 (MH). Kerala: Palakkad District: Panthanthode, 825 m, 21.08. 1966, E. Vajravelu 27574 (CAL \& MH); Thissur District: Parambikkulam submergible area, 607 m, 28.10.1964, K.M. Sebastine 22388 (CAL \& MH); Travancore State, s.d., C.C. Calder \& M.S. Ramaswami 1695 (CAL). Madhya Pradesh: Mandla District: 02.12.1961, J. Joseph 13520 (CAL); Mandla, 500 m, 02.12.1961, J. Joseph 13520 (BSA). Maharashtra: Bombay State, Goregan, 10.12.1956, S.C. Agarwal 1034 (CAL); Radhanagri, 09.12.1956, S.C. Agarwal 1034 (CAL); Sholapur, Oct. 1948, M.P. Srinivas 01 (CAL); Pune District: BSI Garden, 27.09.1966, N.P. Singh 108956 (BSI); Indian Women University, 09.08. 1921, s.coll. s.n. (BSI); Junnar, 14.10.1962, M.Y. Ansari 81984 (CAL); Junnar-JhavleChi-Wadi, 06.10.1965. K. Hemadri 106862 (CAL); Junnar-6 miles away, 08.10.1965, K. Hemadri 106922 (CAL); Kahapur-Haveli Taluka, 13.08. 1964, M.Y. Ansari 99856 (BSI). Rajasthan: Jaisalmer District: 19.09.1956, B.D. Patil 564 (CAL); Jalor District:
Raniwara, 17.08. 1978, B.L. Vyas 6394 (CAL); Pali District: Bijapur, 30.08. 1975, B.V. Shetty 1950 (CAL); Jojawar, 19.11.1974, B.V. Shetty 1517 (CAL). Tamil Nadu: Coimbatore District: s.dat., C.E.C. Fischer 1192 (CAL); 28.08. 1956, K.M. Sebastine 586 (CAL); Anakkatti-Ottakkal Hill near Arnarkad, 23.12.1969, M.V. Viswanathan, 296 (MH); Arachalur, 07.09.1910, C.E.C. Fischer 2151 (FRC); Central Farm, Dec. 1915, s.coll. s.n. (MH); Chinnathadagam, $666 \mathrm{~m}, 22.08 .1956$, K.M. Sebastine 586 (MH); Coimbatore, Feb. 1925, s.coll. 16877 (MH); Cowdally, $\pm 606 \mathrm{~m}, 05.02 .1930, \mathrm{~V}$. Narayanaswami 19484 (MH); Dinhalli, 13.11.06, C.E.C. Fischer s.n. (FRC); Ethanibetta-Kollegal, 1082 m, 04.07.1930, s.coll. 3485 (MH); Hassanur, \pm 914 m, 21.06.1929, K.C. Jacob 18560 (MH); \pm 914 m, 10.03.1931, s.coll. 250 (MH); Hunasikal BettaLokkanahalli-Kollegal, $\pm 914 \mathrm{~m}, 13.01 .1930, T . R$. Naganathan 19458 (MH); Kunjur, \pm 853 m, 27.01.1931, S.R. Raju \& T.R. Naganathan 4970 (MH); Kuridimalai, 600 m, 27.11.1956, K. Subramanyam 1461 (CAL \& $\mathrm{MH}$ ); Poonachi Ghat, 08.10.1901, C.A. Barber 3561 (MH); Ramapuram-Kollegal, 16.12.1924, K.C. Jacob 17349 (MH); Saibaba Colony, 469 m, 15.10.1966, $M$. Chandrabose 28903 (CAL \& MH); Varapalayam-On the way, $666 \mathrm{~m}, 25.07 .1956$, K. Subramanyam 381 $(\mathrm{MH})$; Cuddalore District: Shanikulam, 09.09.1899, s.coll. $873(\mathrm{MH})$; Dindigul District: Kodaikkanal HillsZigzag, 19.12.1905, C.A. Barber 7449 (MH); SirumalaiBase, 28.11.1898, s.coll. s.n (MH); Madras: Sadivayilnear the nursery, 03 Apr. 1963, K.N. Subramanyam 204 (FRC); Madurai District: Kumili-Near Forbay Dam, 750 m, 22.06.1959, K. Subramanyam 8126 (CAL \& $\mathrm{MH})$; Periyakulam, 18.12.1905, C.A. Barber 7446 (MH); Nilgiri District: September 1883, J.S. Gamble 12723 (CAL); 1891, Stahl s.n. (CAL); Gundah River Bank, 01.08. 1975, E. Vajravelu 46410 (MH); KalhathiMasinagudi Road, 1150 m, 23.08. 1970, B.D. Sharma 35739 (MH); Masinagudi, April 1900, C.A. Barber 2667 (MH); Peninsular Indiae Orientalis, 1866, Wight 1038 (CAL); Salem District: Betta Mogilalam-Metagiri Hills, 25.12.1913, s.coll. 9831 (MH); Hosur, 30.12.1916, s.coll. $13984(\mathrm{MH})$; Hosur Cattle Farm, \pm 914 m, 07.06.1930, V. Narayanaswami 2953 (MH); Metagiri, 12.12.1917, s.coll. 15437 (MH); Tirunelveli District: Kalakad to Sengalteri, 23.09.1915, s.coll. 12428 (MH); Mundanthorai Ghat, s.d., C.A. Barber 2773 (MH). Uttarpradesh: Kumaon District: s.d., G. King s.n. (CAL); N.W. Himalaya, Sao Valley, 10.10.1898, J.H. Lace 1869 (CAL).

6. Eragrostis ciliaris (L.) R. Br. in Tuckey, Narr. Exped. Zaire App.: 478. 1818. 
Poa ciliaris L., Syst. Nat., ed. 10, 2: 875. 1759. Lectotype: JAMAICA, Browne s.n. (Herb. Linn. No. 87.66 (LINN), designated by Hitchcock, Contr. U.S. Natl. Herb. 12: 121. 1908.

Annual. Culms $10-25 \mathrm{~cm}$ high, slender, erect to decumbent, geniculate in the lower portion; nodes brownish; internodes $c .2 \mathrm{~cm}$ long with non-viscous glandular ring or spots close below nodes or glands absent. Leaf sheaths $c .1 \mathrm{~cm}$ long, with tubercle based cilia, mouth bearded. Ligule a fringe of cilia. Leaf blades $1.5-2 \times 0.15-2 \mathrm{~cm}$, linear to lanceolate, upper surface scaberulous. Panicles $2-6 \times 1-2.5 \mathrm{~cm}$, spiciform to compact or narrowly open, lanceolate to oblong; primary branches alternate to subwhorled; axils glabrous; pedicels $0.5-1 \mathrm{~mm}$ long, eglandular, at times gland-dotted. Spikelets $1.8-4 \times 1.2-2 \mathrm{~mm}, 5-10-$ flowered, ovate to lanceolate, pale green; rachilla zigzag; florets more or less closely arranged on rachilla, disarticulating from above downwards. Glumes lanceolate, chartaceous, 1-nerved, 1-keeled, scabrous with 2-5 cilia along keel, apex acute; lower glume $c$. $0.5 \times 0.2 \mathrm{~mm}$; upper glume $c .0 .6 \times 0.2 \mathrm{~mm}$. Lemmas $0.8-1.2 \times 0.3-0.6 \mathrm{~mm}$, lanceolate to elliptic, chartaceous, 3-nerved, nerves excurrent, 1-keeled, scabrous with 2-5 longer cilia along keel, apex acute to obliquely truncate. Paleas $0.7-1 \times 0.3-0.4 \mathrm{~mm}$, caducous, elliptic to lanceolate, 2-nerved, 2-keeled, long ciliate along keels, apex acute to obtuse; cilia on palea keels stiff, bulbous based, longer than width of palea body; palea flaps narrower than its body. Anthers 2, c. $0.15 \mathrm{~mm}$ long, purplish. Caryopses $0.3-0.5 \times 0.2-$ $0.3 \mathrm{~mm}$, ovoid to ellipsoid, yellowish brown.

Key to the varieties

1a. Panicles open; branches 1-2.5 cm wide; spikelets scattered on branches ...6.3. var. clarkei 1b. Panicles compact; branches $1-1.3 \mathrm{~cm}$ wide; spikelets clustered on main rachis

2a. Panicles oblong or lanceolate, not lobed

$$
\text { ...6.1. var. ciliaris }
$$

2b. Panicles interrupted, lobed ...6.2. var.brachystachya 6.1. Eragrostis ciliaris var. ciliaris (Fig. 2 \& Plate 1)

Panicles compact, oblong or lanceolate; branches 1$1.3 \mathrm{~cm}$ wide. Spikelets clustered on main rachis.

Flowering and fruiting: Almost throughout the year.

Distribution: INDIA: Almost throughout; tropical and subtropical parts of the world.
Habitat: Usually grows in dry areas, at times also along wet sandy shores, commonly found between 250 and $350 \mathrm{~m}$.

Etymology: The varietal epithet 'ciliaris' means ciliate, alluding to the long-ciliate keels of palea.

Chromosome nos.: $2 \mathrm{n}=40$ (Moffett \& Hurcombe, 1949), 2n = 20 (de Wet, 1960; Mehra \& al., 1968; Dujardin, 1978).

Vernacular names: Burbudi, Tor chandbol, Undarpuncho (Bengali).

Notes: Inflorescence of this taxon is condensed and appears to be woolly because of the densely ciliate palea keels. This can be at times confused with the compact panicle of E. riparia (Willd.) Nees. Most of the specimens kept under the name of E. ciliaris in the herbaria infact belonged to the E. riparia. Major distinction between $E$. ciliaris and E. riparia involved in the number of anthers i.e. 2 in E. ciliaris versus 3 in E. riparia, and cilia on palea keels are prominently bulbous based in E. ciliaris versus that are not bulbous based in E. riparia (Vivek \& al., 2019).

Specimens examined: INDIA. Andhra Pradesh: Nalconda District: Krishna River bank, 16.12.1959, K.M. Sebastine 9819 (CAL \& MH). Gujarat: Sourashtra Coast-Bwark-Thor Jungle, 15.10.1961, T.A. Rao 439 (CAL). Haryana: Hissar District: Rajghat road, 30.11.1961, N.C. Nair 18810 (BSD); Mahendragarh District: Narnaul, 24.10.1962, N.C. Nair 25197 (BSD); Sirsa District: without precise locality, 18.02.1963, N.C. Nair 26060 (BSD); Sonipat District: without precise locality, 15.08. 1962, V.J. Nair 23288 (BSD). Jharkhand: Ramgarh, 01.09.1964, B.M. Wadhwa 5114 (CAL). Madhya Pradesh: Khargaon District: Bistan, 21.01.1990, B.K. Sinha 50345 (BSA); Raigarh District: Dharanjaygarh, 09.11.1971, G. Sengupta 16029 (BSA). Maharashtra: Bombay State-Taranga Hills, 28.01.1957, S.K. Jain 11340 (CAL); Khanajun-Junnar R.F., 10.10.1965, K. Hemadri 106961 (CAL); Kolwam-Mahr, 25.11.1956, s.coll. 9595 (CAL); Naiayangaon, 01.11.1967, K. Hemadri 106989 (CAL). Pujab: Rohtak District: without precise locality, 13.08. 1962, V.J. Nair 23189 (BSD); Khansala District: 14.08. 1962, V.J. Nair 23254 (BSD). Rajasthan: Alwar District: Saruska, 26.10.1983, P.J. Parmar 9601 (CAL); Bikaner District: without precise locality, 11.09.1975, G.P. Roy 2035 (CAL); Churu District: without precise locality, 18.07.1976, G.P. Roy 2696 (CAL); Dhilwara: Umed Sagar, 06.10.1978, A.N. Singh 5757 (CAL); Ganganagar District: without precise locality, 14.11.1976, G.P. Roy 
3881; without precise locality, 08.10.1977, G.P. Roy 4966 (CAL); Jaipur District: Jagatpur, 22.09.1963, S. Sharma 232 (CAL); Jaisalmer District: without precise locality, 24.08. 1976, B.V. Shetty 3308 (CAL); without precise locality, 07.11.1981, R.P. Pandey 7865 (CAL); Jalor District: without precise locality, 14.10.1977, B.L. Vyas 4573 (CAL); without precise locality, 14.08.1978, B.L. Vyas 6359 (CAL); without precise locality, 28.09.1978, B.V. Shetty 6757 (CAL); Jhujhunu District: Lohargal, Sept. 1960, N.C. Nair 2002, 2021 (BSD); Jodhpur District: without precise locality, 09.10.1976, A.N. Singh 3153 (CAL); Kota District: Shahabad, 15.09.1968, R.B. Majumdar 13151 (BSA); Pali District: Erinpura-near Jawai Dam, 01.10.1960, R.S. Rao 66751 (CAL); Udaipur District: Vaghrama, 06.09.1956, S.C. Agarwal 465 (CAL). Tamil Nadu: Kancheepuram District: Mahabalipuram, 27.09.1970, A.R.K. Sastry 7621 (CAL); Ramanathapuram District: Rameshwaram, 22.12.1960, T.A. Rao 590 (CAL); Viluppuram District: Marakkanam Seashore, 19.04.1972, A.K.K. Sastry 9029 (CAL). Uttar Pradesh: Allahabad District: Chathamline, 28.09.1968, T. Rajagopal 4683 (BSA); Kumbha Mela area -Arail, 30.10.2003, K.K. Khanna 58439 (BSA); Dhar District: Dahi, 24.09.1977, O.P. Misra 27767 (BSA); Firozabad District: Shikohabad, 29.01.1961, C.N. Malhotra 13559 (BSD); Saharanpur District: Narsan, Aug. 1985, T.S. Murthy \& A.K. Goel 1863 (BSD).

6.2. Eragrostis ciliaris var. brachystachya Boiss., Fl. Orient. 5: 582. 1884. Type: "Hab. in arenosis littoris Egyptiaco-Arabici inter Kosseir et Ras Benass; Schv. 1091. Belutschia; J.E. Stocks s.n.

Panicles compact, interrupted, almost globular, lobed; branches $1-1.3 \mathrm{~cm}$ wide. Spikelets clustered on main rachis.

Flowering and fruiting: October.

Distribution: INDIA: Gujarat, Haryana, Maharashtra, Rajasthan, Tamil Nadu and Uttar Pradesh; EGYPT.

Specimens examined: INDIA. Gujarat: Mahesana District: 27.12.1977, V. Singh 5476 (CAL); Sourashtra Coast-Okha, 17.10.1961, T.A. Rao 439, 480 (CAL); 16.11.1962, T.A. Rao 1255; 22.11.1963, T.A. Rao 1982 (CAL). Haryana: Gurgaon District: 25.10.1962, N.C. Nair 25250 (BSD); Hissar District: Siwani, 23.10.1967, V.J. Nair 37609 (BSD). Rajasthan: Barmer District: Without precise locality, 15.11.1981, R.P. Pandey 8034 (CAL); Jalor District: Jan. 1971, R.B. Majumdar 165 (CAL); Marwar, 1868, G. King s.n. (CAL); Jhujhunu District: Pilani, Nov. 1961, B.D. Deshpande 6 (BSD);
Jodhpur District: 15.11.1976, B.V. Shetty 308 (CAL). Tamil Nadu: Coimbatore District: Chidambaram Park, 467 m, 04.10.1964, M. Chandrabose 28576 (MH). Uttar Pradesh: Etawah District: Without precise locality, 27.01.1961, C.L. Malhotra 13521 (BSD).

6.3. Eragrostis ciliaris var. clarkei Stapf in Hook.f., Fl. Brit. India 7: 315. 1896. Type: INDIA, Delhi, Clarke s.n. (holo CAL0000002456!).

Panicles open, $1-2.5 \mathrm{~cm}$ wide. Spikelets scattered on branches.

Flowering and fruiting: June.

Distribution: INDIA: Delhi, Karnataka and Rajasthan. Endemic.

Notes: Panicle of this variety is open and also morphologically quite different from that of the other two varieties. However, at times it is confused with $E$. tenella and some forms of $E$. viscosa. But presence of 2 anthers and more prominently bulbous-based and longer cilia on palea keels are main characters to differentiate it from E. tenella and E. viscosa.

Specimens examined: INDIA. Karnataka: Chitradurga District: Hosakere, 13.11.1978, K.P. Sreenath \& S.R. Ramesh 4201 (CAL). Rajasthan: Jalor District: without precise locality, 16.10.1977, B.L. Vyas 5114 (CAL).

7. Eragrostis ciliata (Roxb.) Nees, Agrost. Bras.: 512. 1829.

Poa ciliata Roxb., Fl. Ind. 1: 336. 1820. Lectotype: INDIA ORIENTALIS, N. Wallich 5015 (BM [BM000797909 image!]), designated by Chaisongkrama \& al. (2013).

Poa incurvata Rottler ex Stapf in Hook.f., Fl. Brit. India 7: 313. 1896, nom. inval. Type: INDIA, Rottler s.n. (Wall.,Numer. List: n. 5014).

(Fig. 2 \& Plate 1)

Perennial. Culms $60-120 \mathrm{~cm}$ high, erect or decumbent, geniculate, nodes brownish; internodes $c .14 \mathrm{~cm}$ long. Leaf sheaths $c .5 \mathrm{~cm}$ long, ciliate along one margin, with bearded mouth. Ligule a fringe of cilia. Leaf blades c. $17 \times 0.5 \mathrm{~cm}$, linear to lanceolate, upper surface scabrid. Panicles 3-8 $\times 0.5-1 \mathrm{~cm}$, spiciform, cylindrical, compact, oblanceolate to oblong; primary branches alternate to subwhorled; axils ciliate; pedicels $c .0 .5 \mathrm{~mm}$ long. Spikelets 3.8-5 × 1.5-2.5 mm, 5-10-flowered, ovate to lanceolate, greenish; rachilla zigzag; florets closely arranged on rachilla, disarticulating from above downwards. Glumes linear to lanceolate, chartaceous, margins ciliate, 1-nerved, 1-keeled, scabrid along keel, 
apex acuminate; lower glume $c .1 \times 0.5 \mathrm{~mm}$; upper glume c. $1.3 \times 0.5 \mathrm{~mm}$. Lemmas $1.6-1.8 \times 0.5-0.6 \mathrm{~mm}$, ovate to lanceolate, chartaceous, margins ciliate, 3-nerved, 1-keeled, scabrid along keel, apex acuminate. Paleas 1-1.2 mm long, caducous, oblanceolate to elliptic, 2nerved, 2-keeled, ciliate along keels, apex acute to obtuse; cilia on palea keels bulbous-based, stiff, equaling to or longer than width of palea body; palea flaps almost equal in width to its body and closely ciliate along margins. Anthers 2, c. $2 \mathrm{~mm}$ long. Caryopses $0.4-0.5 \times 0.2-0.3 \mathrm{~mm}$, ovoid to ellipsoid, deep brown.

Flowering and fruiting: Almost throughout the year.

Distribution: INDIA: Andhra Pradesh, Bihar, Gujarat, Madhya Pradesh, Maharashtra, Odisha, Tamil Nadu and West Bengal; SOUTHEAST ASIA.

Habitat: Open places and waste land.

Notes: The name Eragrostis ciliata (Roxb.) Nees was based on Poa ciliata Roxb. (1820). The name was validated by Wight \& Arnott in Wight's Catalogue of Indian Plants 106 (1834) without knowing that the earlier combination made by Nees (1829) was a legitimate one thus the combination had priority over the validation of Nees (Noltie, 2005).

Specimens examined: INDIA. Andhra Pradesh: East Godavari district: Annavaram, 17.01.1916 (MH); Kothapalli, 05.09.1907, C.A. Braber 8273 (MH); Srikakulam District: Palasa, 20.11.1955, M.P. Guha 193; 20.11.1955, B.D. Patil 181 (CAL); Visakhapatnam district: Bhupathipaliam, 27.09.1920, $V$. Narayanaswami 256 (CAL); Chidepallem near, 26.02.1947, V. Narayanaswami 663 (CAL); Itchapuram R.F., 08.12.1923, K.C. Jacob 17097 (MH); Maripalli, 13.10.1930, V. Narayanswami 4597 (MH). Chhattisgarh: Bastar district: Indravati Tiger Reserve, 13.02.1988, Anand Kumar 15584 (CAL); Kutunsar, 19.02.1963, G. Panigrahi 1781 (BSA). Madhya Pradesh: Narsingpur district: Naringhpur, 18.03.1943, K. Biswas 6129 (CAL); Raoghat, 15.02.1961, N.P. Balakrishnan \& A.N. Henry 12145 (CAL \& MH); Umaria District: Bandhavgarh National Park, Shadol-BHP, 14.01.1997, S.K. Srivastava 45689 (BSA). Tamil Nadu: Tirunelveli district: Mundanthorai Ghat, 14.05.1901, C.A. Barber 2759 (MH); 22.09.1943, D. Daniel \& S.R. Raju 20431 $(\mathrm{MH})$; Peninsula Ind. Orientalis, 1841, Wight 3330 (CAL).

8. Eragrostis coarctata Stapf in Hook.f., Fl. Brit. India 7: 313. 1896. Type: INDIA, Upper Gangetic Plain, Thomson s.n.; Sikkim, Behar, Chittagong, Arracan and
Myanmar. Central Provinces \& Chota Nagpore, Clarke s.n.

(Fig. 2 \& Plate 1)

Perennial. Culms 10-70 cm high, decumbent, geniculate; nodes brownish; internodes $c .6 \mathrm{~cm}$ long. Leaf sheaths c. $4 \mathrm{~cm}$ long, ciliate along one margin, mouth bearded. Ligule a fringe of cilia. Leaf blades $c$. $15 \times 0.4 \mathrm{~cm}$, linear to lanceolate, upper surface scaberulous. Panicles 5-15 × 1-2.5 cm, spiciform; primary branches alternate to subwhorled; axils longciliate; pedicels $0.3-8 \mathrm{~mm}$ long. Spikelets $2-4.5 \times 1-2$ $\mathrm{mm}, 4-12$-flowered, lanceolate to oblong, greenish; rachilla zigzag; florets closely arranged on rachilla, disarticulating from above downwards. Glumes ovate to lanceolate, chartaceous, margins ciliate, 1-nerved, 1-keeled, scabrid along keel, apex acute; lower glume $0.8-1.1 \times 0.2-0.3 \mathrm{~mm}$; upper glume $1-1.1 \times 0.3-0.4$ $\mathrm{mm}$. Lemmas $1.3-1.7 \times 0.4-0.5 \mathrm{~mm}$, elliptic to lanceolate, chartaceous, margins ciliate, 3-nerved, 1keeled, scaberulous along keel, apex acuminate. Paleas $1.2-1.4 \times 0.3-0.4 \mathrm{~mm}$, caducous, elliptic to oblanceolate, chartaceous, 2-nerved, 2-keeled, ciliate along keels, apex acute to obtuse, at times fimbriate; cilia on palea keels equaling to or more than width of palea body; palea flaps almost equal in width to its body and ciliate along margins. Anthers 3, c. $2 \mathrm{~mm}$ long. Caryopses $0.4-0.5 \times 0.2-0.3 \mathrm{~mm}$, ovoid to ellipsoid, yellowish brown.

Flowering and fruiting: Almost throughout the year.

Distribution: INDIA: Andhra Pradesh, Assam, Bihar, Jharkhand, Karnataka, Madhya Pradesh, Maharashtra, Odisha, Rajasthan, Sikkim, Tamil Nadu, Uttar Pradesh and West Bengal; BANGLADESH and MYANMAR.

Habitat: Grows on sandy soils, also on roadsides, between 500 and $1000 \mathrm{~m}$.

Notes: Panicles of E. coarctata is more or less contracted with the possibility of it being confused with that of $E$. riparia. But margins of glumes and lemmas are ciliate in E. coarctata and eciliate in E. riparia. Moreover, E. coarctata is an eglandular species.

Specimens examined: INDIA. Andhra Pradesh: East Godavari District: without precise locality, 03.01.1902, Bourne 3404 (CAL); Kuntada R.F., Maredumilli, 500 m, 16.02.1994, M. Mohanan 101241 (MH); Mudurlanka, Marudamilli, 500 m, 20.10.1994, M. Mohanan 102567 (MH); Samalkota, 27.08. 1902, C.A. Barber 4537 (MH); Sirivaka, 30.11.1902, C.A. Barber 4987 (MH); Ibid., 08.12.1902, C.A. Barber 5256 (MH); Karimnagar District: Aklaspur, 27.07.1964, G.V. 


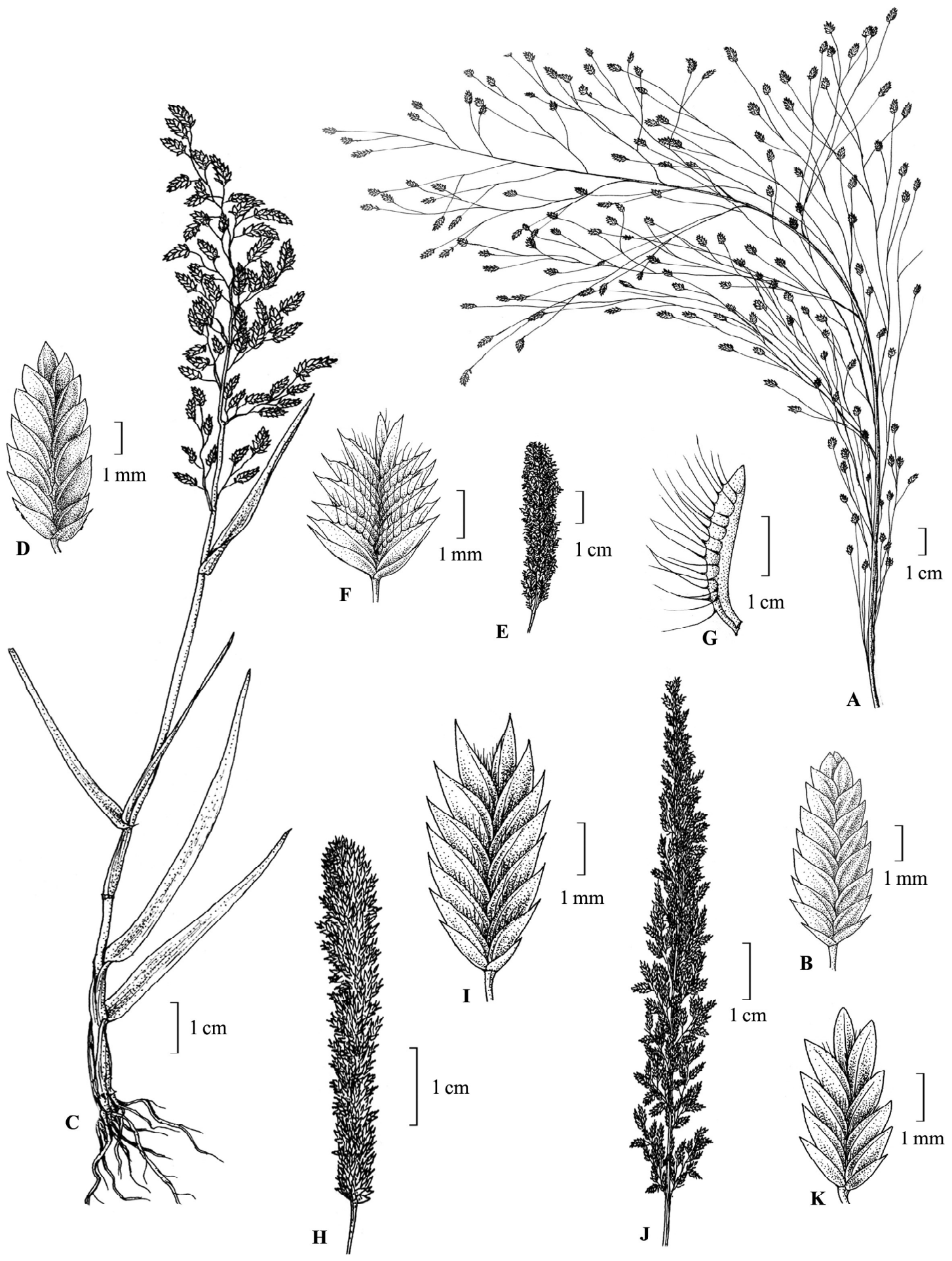

Fig. 2. Eragrostis burmanica Bor (A-B). A. Inflorescence; B. Spikelet. E. cilianensis (All.) Vignolo Lut. ex Janch.; (C-D). C. Habit; D. Spikelet. E. ciliaris (L.) R. Br. var. ciliaris; (E-G). E. Inflorescence; F. Spikelet; G. Palea-lateral view. E. ciliata (Roxb.) Nees; (H-I). H. Inflorescence; I. Spikelet. E. coarctata Stapf.; (J-K). J. Inflorescence; K. Spikelet. 
Subbarao 20236 (CAL); Khammam District: Lakshmipuram Forest, $\pm 250 \mathrm{~m}, 21.11 .1993, R$. Chandrasekaran 98910 (MH); Krishna District: Allur, 19.08.1907, C.A. Barber 7940 (FRC); Nellore District: Kollur, 15.12.1917, C.E.C. Fischer 4222 (FRC); Srikakulam District: Korada R.F., 125 m, 12.05.1979, G.V. Subbarao 62382 (MH); Visakhapatnam District: Araku Valley, 15.09.1961, N.P. Balakrishnan 587 (CAL); Barnakonda R.F., 09.12.1923, K.C. Jacob 17281 (MH). Bihar: Champaran District: Without precise locality, 14.04.1963, K. Thothathri 10087 (CAL). Chhattisgarh: Bastar District: Bailadilla, 15.02.1963, G. Panigrahi 7000 (BSA); Chotadongar Nursery, 21.01.1990, G.P. Roy 65020 (BSA); Keskal, 567 m, 17.11.1958, K. Subramanyam 7124 (CAL \& MH); Kanger Valley National Park, 15.01.2004, Ajay Kumar 57432 (BSA). Jharkhand: Ranchi District: s.d., B.D. Patil 231 (CAL); Koil Karo River, 15.02.1981, K.C. Malik 9702 (CAL); Gaya District: 28.12.1876, C.B. Clarke 31842 (CAL); Singhbhum District: Goelkera, 27.12.1960, G.V.S. Rao 23726 (CAL); Singhbhum, 24.12.1902, H.H. Haines 545 (CAL). Madhya Pradesh: Bilaspur District: Bilaspur, Feb. 1972, S.K. Murti 15339 (CAL); Korbi, 10.02.1972, G. Panigrahi 15339 (BSA); Chhindwara District: Nagdwari, 01.02.2004, Parul Sharma 59645 (BSA); Korba District: Gevera, 17.11.2006, B.K. Shukla 67380 (BSA); Korba to Kudura, G. Panigrahi 16807 (CAL); Koroi, Feb. 1972, S.K. Murti 15337 (CAL); Manikpur, 29.03.2006, B.K. Shukla 65031 (BSA); Raigarh District: Barumkila, 02.02.1974, N.C. Rathakrishnan 19629 (BSA); Dharanjaygarh, 09.11.1971, G. Sengupta 16029 (BSA); Silot Block, Gargode Range, 09.11.1971, G. Sengupta 16071 (BSA). Odisha: Cuttack District: 22.05.1967, M. Ghosh 227 (CAL); Ganjam District: Boragharo, 20.08. 1931, V. Narayanaswami 5963 (MH); Koraput District: Jamunda, Nov. 1885, Prain s.n., CAL Acc. No. 541184 (CAL); Malkangiri District: Orkel, 07.11.1963, D.C.S. Raju 1037 (CAL); Sundargarh District: Panposh, 02.10.1986, D. Namhata 304 (CAL). Sikkim: East District: Chaisay, on the way to Rongli, $\pm 1219 \mathrm{~m}$, 07.12.1980, P. Chkrabarty 1022 (BSHC); West Sikkim: Naya Boyer to Somboriya Road, 11.02.1996, B.K Shukla \& S.K. Rai 18638 (BSHC). Uttar Pradesh: Bahraich District: Bahraich, Feb. 1959, M.A. Rao 8204 (BSD); Ibid.,.09.1959, M.A. Rao 8205 (BSD); Ibid., 09.02.1959, M.A. Rao 8194 (BSA \& BSD); Bijnor District: Balawali, 12.03.1958, Y.K. Sarin 5038 (BSD); Chandpur District: 05.03.1962, C.L. Malhotra 20236 (BSD); Chitrakoot District: Banda, 14.11.1957, M.A. Rao 3761 (BSD); Pilibhit District: Dhanaraghat, 19.10.1970, C.L.
Malhotra 42840 (BSD); Madhotanda, 20.10.1970, C.L. Malhotra 42860 (BSD); Lucknow District: Carlton Hotel, s.dat., E.K. Janakiammal 68 (BSA). West Bengal: Birbhum District: 16.12.1969, R.K. Basak 1241 (CAL); 10.03.2008, A.K. Sinha 43310 (CAL); Shantiniketan, 28.01.1956, S.K. Mukherjee 4101 (CAL); Midnapur District: 16.05.1963, G. Sen Gupta 397 (CAL); Baura, 03.03.1976, Sudarsan Maji 2909 (CAL); Bhakra, 06.06.1975, Sudarsan Maji 1553 (CAL); Jhargam, 22.02.1976, Sudarsan Maji 2645 (CAL); Junput, 05.12.1975, Sudarsan Maji 2532 (CAL); Purulia District: 20.04.1968, K.C. Malick 539 (CAL); Chota Nagpur,.12.1880, J.S. Gamble 8986 (CAL); Ibid., 12.11.1883, C.B. Clarke 34086 (CAL); Ibid., Jan. 1903, Prain 61 (CAL).

9. Eragrostis collinensis Vivek, G.V.S. Murthy \& V.J. Nair in Indian J. Forest. 36(3): 401. 2013. Type: INDIA, Tamil Nadu, Nilgiris District, Avalanche, 1925 m, 14 October 1972, K. Vivekanandan 42951 (holo CAL!, iso $\mathrm{MH}$ !).

(Fig. 3 \& Plate 1)

Perennial. Culms $10-38 \mathrm{~cm}$ high, erect; nodes brownish; internodes $2-3.5 \mathrm{~cm}$ long. Leaf sheaths $c .4$ $\mathrm{cm}$ long, margins glabrous or finely ciliate, with bearded mouth. Ligule c. $0.2 \mathrm{~mm}$ long, a ciliate rim with narrow membrane at base. Leaf blades $10-30 \times$ $0.5-1 \mathrm{~cm}$, linear to lanceolate or flat, upper surface scabrid to sparsely ciliate. Panicles 5-9 $\times 1.5-4 \mathrm{~cm}$, open, ovate; primary branches alternate, spreading; axils glabrous; pedicels up to $3 \mathrm{~mm}$ long. Spikelets 4$10 \times 3-4 \mathrm{~mm}, 3-39$-flowered, ovate to oblong or elliptic, margins serrate in appearance, purplish to dark pink or chocolate brown in colour; rachilla zigzag with internode up to $1 \mathrm{~mm}$ long; florets loosely arranged on rachilla, disarticulating from below upwards. Glumes unequal, lanceolate, chartaceous, 1-nerved, 1-keeled, scabrous along keel above middle, apex acute; lower glume 1.3-1.7 × c. $0.3 \mathrm{~mm}$; upper glume 1.9-2.1 $\times$ c $0.4 \mathrm{~mm}$. Lemmas $1.9-2 \times 0.4-0.8 \mathrm{~mm}$, lanceolate to elliptic, chartaceous, 3-nerved, lateral nerves straight, 1-keeled, scabrid along keel towards apex, apex acute. Paleas 1.5-1.9 $\times 0.4-0.8 \mathrm{~mm}$, caducous, elliptic to lanceolate, 2-nerved, 2-keeled, scaberulous along keels, apex acute to obtuse; palea flaps narrower than its body. Anthers 3, 0.5-0.8 mm long, deep purple. Caryopses up to $0.7 \times 0.4 \mathrm{~mm}$, obovoid to narrowly ellipsoid, reddish brown.

Flowering and fruiting: Throughout the year.

Distribution: INDIA: Kerala and Tamil Nadu. Endemic. Habitat: Hills, 1000-2200 m. 

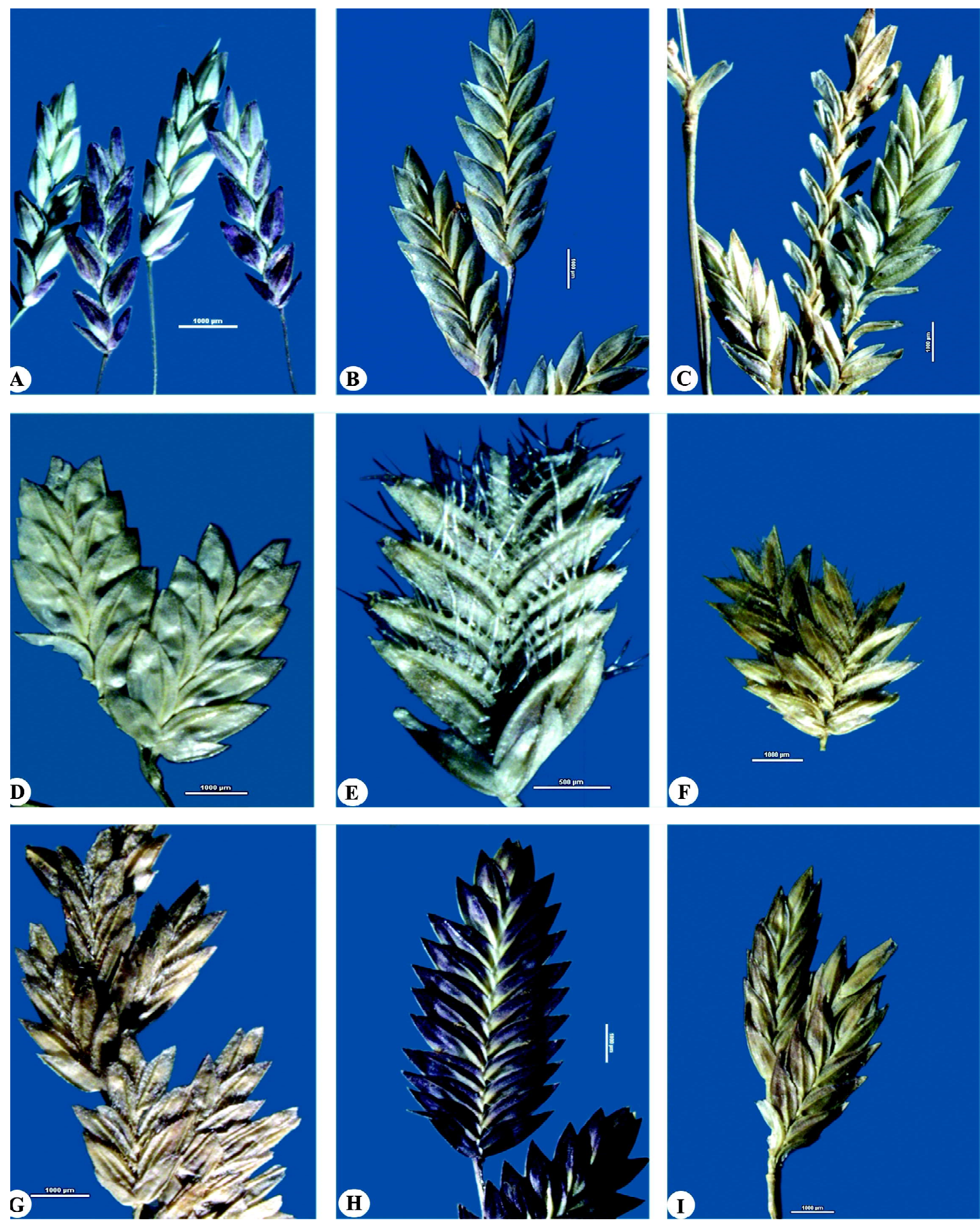

Plate 1. Spikelets of : A. Eragrostis aspera (Jacq.) Nees; B. E. atrovirens (Desf.) Trin. ex Steud.; C. E. brownii (Kunth) Nees; D. E. cilianensis (All.) Vignolo Lut. ex Janch; E. E. ciliaris (L.) R. Br. var. ciliaris; F. E. ciliata (Roxb.) Nees; G. E. coarctata Stapf; H. E. collinensis Vivek, G.V.S. Murthy \& V.J. Nair; I. E. cumingii Steud. 
Etymology: The specific epithet collinensis (collina = pertaining to hills), refers to the distribution of the species on hills.

Notes: This species is distinguished from E. unioloides by its much broader spikelets and loosely arranged florets on rachilla, erect lemmas, wingless palea and 3 longer and broader anthers (Vivek \& al., 2013b).

Specimens examined: INDIA. Kerala: Idukki District: Munnar-Travancore, 30.05.1944, K.C. Jacob 20485 (MH); Kollam District: Anathode, 1100 m, 10.11.1975, K. Vivekananthan 46598 (MH); Kottayam District: Devicolam-Travancore, 02.06.1944. K.C. Jacob 20516 (MH); Upper Vagavurrai, $1800 \mathrm{~m}, 08.08$. 1967, B.V. Shetty 28336 (MH). Tamil Nadu: Coimbatore District: Anamali Hills, Konalar, 1925 m, 17.11.1980, M. Chandrabose 69001 (MH); Valparai, $1219 \mathrm{~m}$, 03.03.1942, S.R. Raju 20364 (MH); Nilgiris District: Avalanche, $2000 \mathrm{~m}, 14.06 .1970$, B.V. Shetty 34230 (MH); Coonoor, 1700 m, 28.07.1957, K.M. Sebastine 4079 (MH); Hulical Droog, 1733 m, 27.08.1957, K.M. Sebastine 4131 (MH); Pakasura Fort, $2066 \mathrm{~m}$, 07.12.1957, K.M. Sebastine 4809 (MH); Ootacamund Lake, 2194 m, 15.09.1930, V. Narayanaswami 4373 (MH); Nanjanad, 2010 m, 07.07.1970, J.L. Ellis 34460 (MH); Pykara bank of river, $2133 \mathrm{~m}, 12.09 .1930, \mathrm{~V}$. Narayanaswami 4240 (MH); Sullikodu R.F., Kotagiri, 2200 m, 28.07.1970, E. Vajravelu 35141 (MH); Salem District: Balmadies, Yercaud, 1300 m, 05.11.1965, A.V.N. Rao 26970 (MH); Kauveri Peak, Yercaud, 1700 m, 04.11.1968, D.B. Deb 31301 (CAL \& MH).

10. Eragrostis cumingii Steud., Syn. Pl. Glumac. 1: 266. 1854. Type: PHILIPPINES, Luzon, 1841, Cuming 1104, designated by Lazarides in Austral. Syst. Bot. 10: 109. 1997 (lecto K000290379, isolecto G00301770, P00622473, P00622472 images!).

Eragrostis distans Hack., Publ. Bur. Sci. Gov. Lab. 35: 81. 1906. Type: PHILIPPINES: Kias, Benguet, Luzon, June, 1904, Elmer 6608 (holo W 17346).

(Fig. 3 \& Plate 1)

Annual. Culms 10-50 cm high, erect or decumbent, slender; nodes brownish; internodes $2-5 \mathrm{~cm}$ long, glabrous. Leaf sheaths $1.5-4 \mathrm{~cm}$ long, glabrous or sparsely bulbous-based ciliate, mouth bearded. Ligule a fringe of cilia. Leaves mostly basal, filiform; blades 5$10 \mathrm{~cm}$ long, linear to lanceolate, upper surface sparsely ciliate. Panicles 4-10 $\times 1-2 \mathrm{~cm}$, open, elliptic to oblong, with spikelets clumped along branches; primary branches $1-3 \mathrm{~cm}$ long, fascicled; axils densely ciliate; pedicels $0.5-1.5 \mathrm{~mm}$ long. Spikelets $5-10 \times 1-2.3 \mathrm{~mm}$, 10-30-flowered, lanceolate to oblong, apex sharply acute, greenish to pinkish; rachilla zigzag; florets firmly arranged on rachilla, disarticulating from below upwards. Glumes linear to lanceolate, chartaceous to subcoriaceous, 1-nerved, 1-keeled, scabrous along keel, apex acute to acuminate; lower glume 1-1.5 ×0.3-0.5 $\mathrm{mm}$; upper glume, $1.5-2 \times 0.5-0.7 \mathrm{~mm}$. Lemmas $1.5-$ $1.8 \times 0.6-1 \mathrm{~mm}$, ovate to lanceolate, subcoriaceous, 3 nerved, lateral nerves nearly straight, 1-keeled, scabrous along keel, apex acute. Paleas 1-1.3 × 0.2-0.4 mm, persistent, elliptic to oblanceolate, narrowly curved, 2 nerved, 2-keeled, ciliolate along keels, apex acute to obtuse; palea flaps narrower than its body. Anthers 3, $c$. $0.1 \mathrm{~mm}$ long, purplish. Caryopses $c .0 .5 \times 0.3 \mathrm{~mm}$, ovate to globose or orbicular, brownish.

Flowering and fruiting: June-February.

Distribution: INDIA: Andaman and Nicobar Islands and Kerala; AUSTRALIA, BHUTAN, CHINA, JAPAN, MALESIA, MYANMAR TO VIETNAM, PHILIPPINES, introduced in USA.

Habitat: Grows in clayey soils of seasonally wet places, and in coastal areas.

Notes: So far, this species is reported in India from Andaman Islands and Kerala (Vivek \& al., 2020). It has close morphological resemblance with E. zeylanica Nees \& Mey. but differs from it in having wiry culms, shorter and narrower spikelets and lemmas. The number of florets is also comparatively less in E. cumingii.

Specimens examined: INDIA. Andaman and Nicobar Islands: Nicobar Islands, s.d., S. Kurz s.n. (CAL); South Andaman, Beadonabad, 30.11.1973, N.P. Balakrishnan 661 (CAL, PBL); Nayasahar, 11³4.447' N, 09240.470' E, 5 m, 04.03.2019, C.P. Vivek 33203 (PBL); Sippighat, $11^{\circ} 35.543^{\prime} \mathrm{N}, 092^{\circ} 40.497^{\prime} \mathrm{E}, 3 \mathrm{~m}, 25.01 .2019$, C.P. Vivek 33202 (PBL); Port Blair, 3 m, 12.01.1951, K. Thothathri 9042 (CAL, MH, PBL). Kerala: Kannur District: Chandanathode, 825 m, 17.06.1979, V.S. Ramachandran $62649 \quad$ (CAL, MH); Thiruvananthapuram District: Veli, 30 m, 30.07.1979, M. Mohanan 63327 (CAL, MH).

11. Eragrostis curvula (Schrad.) Nees, Fl. Afr. Austral. Ill.: 397. 1841.

Poa curvula Schrad., Gott. Gel. Anz. 3: 2073. 1821. Type: SOUTH AFRICA, Cape Province: Cape of Good Hope, Hesse s.n. in Herb. Trinius 2327.1, lower middle specimen (holo \& iso LE).

Eragrostis robusta Stent in Bothalia 2: 288. 1927. Type: 


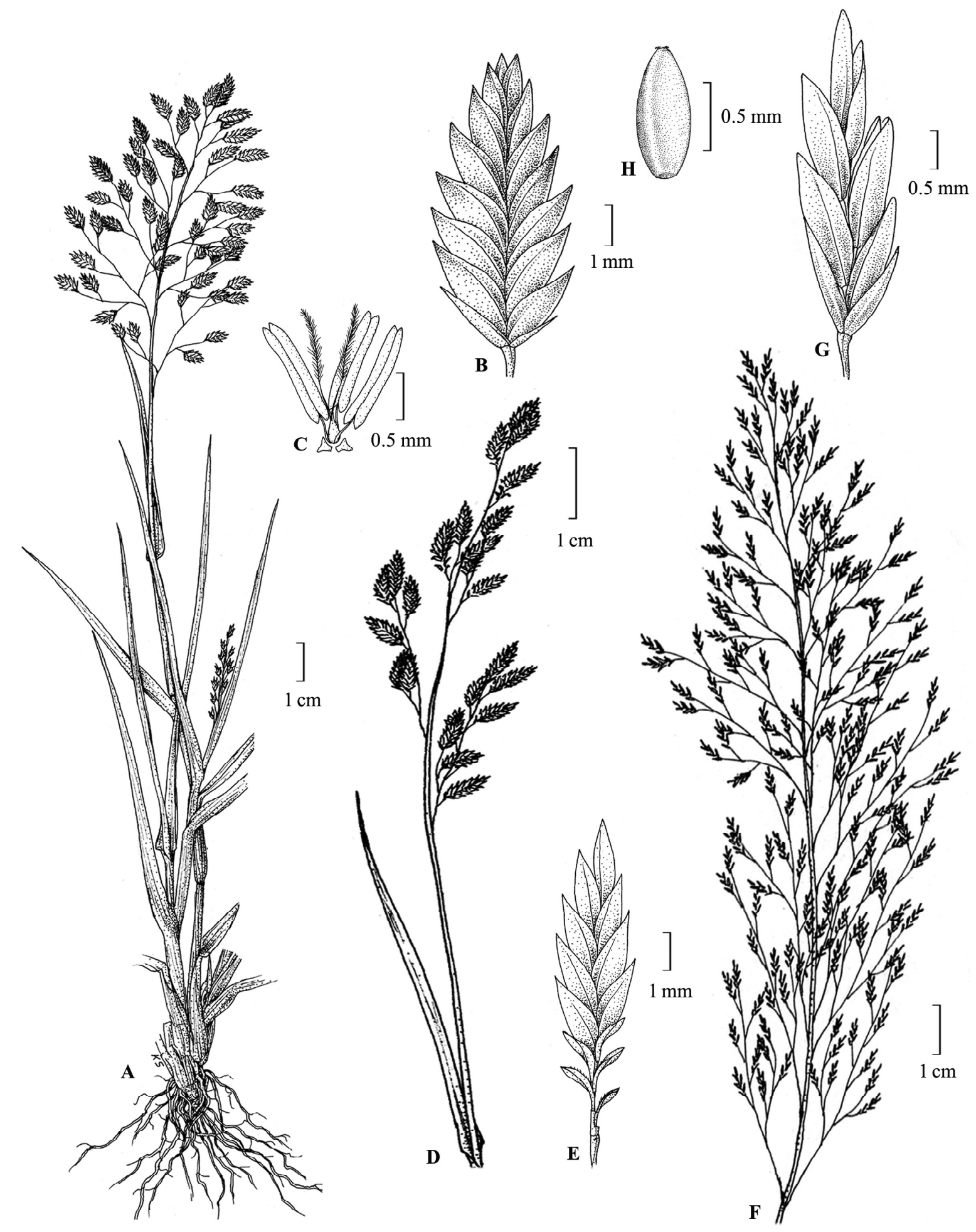

Fig. 3. Eragrostis collinensis Vivek, G.V.S. Murthy \& V.J. Nair (A-C). A. Habit; B. Spikelet; C. Lodicules, Stamens \& Pistil. E. cumingii Steud. (D-E). D. Inflorescence; E. Spikelet. E. curvula (Schrad.) Nees (F-H). F. Inflorescence; G. Spikelet; H. Caryopsis. 
SOUTH AFRICA, Pietermmaritzburg, 28 March 1917, Angus M. 2 (iso PRE0029198, image!).

(Fig. 3 \& Plate 2)

Perennial. Culms 30-120 cm high, densely tufted, erect; nodes brownish; internodes $c .25 \mathrm{~cm}$ long, glabrous. Leaf sheaths $10-25 \mathrm{~cm}$ long, hard and yellowish, villous hairy at young stage, glabrous when mature, mouth bearded. Ligule a ciliate rim with narrow membrane at base. Leaf blades $10-35 \times 0.2-0.5 \mathrm{~cm}$, linear to filiform, drooping, margins inrolled, upper surface scaberulous, apex attenuate to a point. Panicles $6-40 \times 8-16 \mathrm{~cm}$, broadly ovate, lax, loose and spreading or narrow to contracted; branches up to $16 \mathrm{~cm}$ long, alternate to subwhorled, rigid, with or without glandular patches; pedicels 1-5 mm long, angled, scabrous along angles. Spikelets 4-10×1-1.6 mm, 4-13-flowered, lanceolate to oblong, green to grey coloured; rachilla persistent below but fragile above, zigzag; florets more or less closely arranged on rachilla, disarticulating from below upwards. Glumes lanceolate, chartaceous, 1-nerved, 1keeled, scabrous or glabrous along keel, apex subacute; lower glume $0.8-1.8 \times c$. $0.5 \mathrm{~mm}$; upper glume $1.5-2.2$ $\times$ c. $0.6 \mathrm{~mm}$. Lemmas $1.8-2.6 \times c .1 \mathrm{~mm}$, narrowly ovate to lanceolate or elliptic, chartaceous, 3-nerved, 1keeled, glabrous or scabrous along keel, apex acute. Paleas c. $2 \times 0.4 \mathrm{~mm}$, persistent, elliptic, 2-nerved, 2keeled, scaberulous along keels above middle, apex obtuse to truncate; palea flaps almost equal in width to its body. Anthers 3, c. $1 \mathrm{~mm}$ long, yellowish to purplish. Caryopses $0.8-1 \times 0.2-0.4 \mathrm{~mm}$, ellipsoid, obtuse or truncate, dorsally compressed to slightly grooved, yellowish brown.

Flowering and fruiting: May-January.

Distribution: INDIA: Rajasthan, Sikkim and Tamil Nadu; Native to SOUTH AFRICA. introduced in KENYA, BOTZWANA, MOZAMBIQUE, MYANMAR, MALESIA, TANZANIA, ZAMBIA and ZIMBABWE.

Habitat: Introduced and naturalized in hilly areas.

Chromosome nos.: 2n = 40 (Nielsen, 1939; Mehra \& al., 1968), $2 \mathrm{n}=50$ (de Wet, 1954), $2 \mathrm{n}=70$ (Pienaar, $1955), 2 \mathrm{n}=42,63,80$ (Roy, 1984), $2 \mathrm{n}=20,60$ (Halvorsen \& Guertin, 2003).

Notes: This polymorphic species is often referred as a member of E. curvula complex and ecotypes with intermediate characters are often seen, thus bringing much confusion in the identity (Covas, 1991; Voigt \& al., 2004). In India, the glandular as well as eglandular forms of this species with much contracted or loose panicle are seen.

Specimens examined: INDIA. Sikkim: Gangtok District: Forest Department, 14.07.1980, B.K. Das 402 (BSHC). Tamil Nadu: Nilgiris District: Nanjanad, 28.01.1952. D. Daniel Sunder Raj s.n. (MH); Sheep farm Ooty, September 1956, Vet. Asst. Surgeon s.n. (MH). Uttarakhand: Tehri District: Dhanaulti, $2318 \mathrm{~m}$, 07.06.1978, A.K. Goel 64126 (BSD).

12. Eragrostis deccanensis Bor, Grass. Burma Ceylon India Pakistan: 507. 1960.

Dactylis phleoides J.G. Klein ex Steud., Nomencl. Bot., ed. 2, 1: 478. 1840.

Eragrostis phleoides Stapf in Hook.f., Fl. Brit. India 7: 313. 1896, non Hillebr. 1888.

Eragrostis spicata Jedwabn. in Bot. Archiv. 5: 185. 1924, nom. illeg., non Vasey 1891. Type: INDIA: Madras, Narayanaswami 3027; Madras; Numer. List [Wallich] no. 5015 (holo K000643390 image!, iso CAL0000002457!).

(Fig. 4 \& Plate 2)

Perennial. Culms 30-100 cm high, geniculate; nodes brownish; internodes $c .8 \mathrm{~cm}$ long. Leaf sheaths $c .4 \mathrm{~cm}$ long, tubercle-based ciliate, mouth bearded. Ligule a fringe of cilia. Leaf blades $4-12 \mathrm{~cm}$ long, linear to lanceolate, margins and both surfaces tubercle based ciliate. Panicles 3-10 × 1.2-1.5 mm, spiciform with clustered spikelets; branches very short, alternate to subwhorled; axils long ciliate. Spikelets 6-8 $\times 2-3 \mathrm{~mm}$, 10-16-flowered, ovate to lanceolate; rachilla zigzag; florets closely arranged on rachilla, disarticulating from above downwards. Glumes ovate to lanceolate, subcoriaceous, margins ciliate, 1-nerved, 1-keeled, scabrous along keel, apex acute; lower glume 1.2-1.5 $\times$ 0.3-0.4 mm; upper glume $1.8-2 \times 0.4-0.5 \mathrm{~mm}$. Lemmas $2.5-3 \times 0.8-1 \mathrm{~mm}$, ovate to lanceolate, sub-coriaceous, margins ciliate, 3-nerved, 1-keeled, scabrous along keel, apex subacute to mucronate. Paleas $1.8-2 \times 0.4-$ $0.6 \mathrm{~mm}$, caducous, oblanceolate to elliptic, 2-nerved, 2-keeled, ciliate and slightly winged along keels, apex acute to obtuse; cilia on palea keels shorter than width of palea body; palea flaps almost equal in width to its body. Anthers 3, c. $3 \mathrm{~mm}$ long. Caryopses $0.4-0.9 \times$ 0.3-0.5 mm, ovoid to ellipsoid, yellowish brown.

Flowering and fruiting: September-October.

Distribution: INDIA: Andhra Pradesh, Karnataka, Kerala, Odisha and Tamil Nadu. Endemic.

Habitat: Grows in hilly tracks. 
Notes: The cylindrical and condensed panicle of $E$. deccanensis is difficult to distinguish from that of $E$. ciliata. Texture of individual spikelet of E. deccanensis, however is more leathery when compared with E. ciliata. Further, cilia along the lemma margins of E. deccanensis are shorter, weaker and more closely arranged and palea keels are narrowly winged versus the cilia of lemma margins are longer, stiffer and distantly arranged and palea keels are wingless in E.ciliata.

Specimens examined: INDIA. Andhra Pradesh: Chitoor District: Irumalai Hills, 12.10.1938, K.C. Jacob 471 (MH). Karnataka: Bangalore, 04.11.1890, s.coll. 535 (MH); Bangalore, 09.1910, A. Meebold 11349 (CAL). Tamil Nadu: Krishnagiri District: Hosur Cattle Farm, 08.06.1930, V. Narayanaswami 3027 (MH).

13. Eragrostis ferruginea (Thunb.) P. Beauv., Ess. Agrostogr. 71, 162, 174.

Poa ferruginea Thunb. in Murray, Syst. Veg., ed. 14: 114. 1784. Type: JAPAN, Herb. Thunberg 2234 (holo UPS, Kew negative no. 869, IDC microfiche 94/20).

(Fig. 4 \& Plate 4)

Perennial. Culms $30-110 \mathrm{~cm}$ high, tufted or single, erect or geniculate; nodes brownish; internodes $c .5$ $\mathrm{cm}$ long, glabrous. Leaf sheaths ciliate along one margin, mouth bearded. Ligule a fringe of cilia. Leaf blades $4-40 \times 0.2-0.6 \mathrm{~cm}$, linear to lanceolate, upper surface sparsely hairy or glabrous, ciliate towards base, lower surface glandular on nerves. Panicles 15-40 $\times$ 4-15 cm, large and open; branches c. $9 \mathrm{~cm}$ long, glandular; axils glabrous. Spikelets $5-10 \times 2-2.5 \mathrm{~mm}$, 4-10 (-20)-flowered, oblong to lanceolate, grey to green or purplish black; florets more or less closely arranged on rachilla, disarticulating from below upwards. Glumes lanceolate, chartaceous, 1-nerved, 1keeled, scabrous along keel, apex acute to acuminate; lower glume 1.4-2 mm long, upper glume $2-3 \mathrm{~mm}$ long. Lemmas $2.4-3 \times$ c. $1.2 \mathrm{~mm}$ long, ovate to lanceolate or oblong, chartaceous, 3-nerved, 1-keeled, scabrous along keel towards apex, apex obtuse to narrowly acute. Paleas 2-2.2 $\mathrm{mm}$ long, persistent, oblanceolate to elliptic, 2-nerved, 2-keeled, scaberulous to ciliolate along keels above middle, apex obtuse; palea flaps almost equal in width to its body. Anthers 3, c. 0.1 $\mathrm{mm}$ long, brownish. Caryopses 0.7-1.5 × c. $0.4 \mathrm{~mm}$, rectangular to prismatic, dorsally flattened to grooved, truncate at both ends, dark reddish.

Flowering and fruiting: August-September.
Distribution: INDIA: Sikkim (Bor, 1960); CHINA, JAPAN and TIBET.

Habitat: Grows in high altitude meadows.

Chromosome no.: 2n = 80 (Tateoka, 1953, 1954, 1967).

Notes: Bor (1960) included it based on a collection from Sikkim (Sikkim, Hook.f. s.n.). But, during the present study the specimen could not be traced in any Indian herbaria. However, as this species occurs in the Eastern Himalayas, and neighboring countries such as Tibet, Nepal and Myanmar, there is every possibility of its occurrence in India, especially in the northeastern Himalayan states thus it is included in the present study. The description and illustration provided here are based on the Chinese and Japanese collections housed at CAL.

Specimens examined: CHINA. 1907, Kurz s.n. (CAL); Thangai 548 (CAL); Central China, 1885, A.N. Henry 4809 (CAL); Koera Septentrionalis, 1897, V. Komorov 174, 2743, 4288 (CAL). JAPAN. Koshigaya in Musashi, 26.09.1950, J. Ohwi 96 (CAL).

14. Eragrostis gangetica (Roxb.) Steud., Syn. Pl. Glumac. 1: 266. 1854.

Poa gangetica Roxb., Fl. Ind. 1:341. 1820. Type: INDIA, Banks of Ganges, Roxburgh s.n. (holo CAL!, K, iso BM image!).

Eragrostis stenophylla Hochst. ex Miq., Anal. Bot. Ind. 2: 27. 1851. Type: INDIA, prope urbem Mangalore, Metz no. 664 (holo L).

Eragrostis dayanandanii Ravich. \& al. in Kew Bull. 51: 155. 1996. Type: INDIA, Tamil Nadu, Chengalpattu District, 24 January1992, Azhakanandam, Krishnan, Ravichandran \& Samson 235 (holo MH!, iso K, Madras Christian College Herbarium, Chennai).

(Fig. 4 \& Plate 4)

Annual. Culms $40-50 \mathrm{~cm}$ high, tufted, geniculately ascending; nodes brownish; internodes 6-9 $\mathrm{cm}$ long. Leaf sheaths 3-7 cm long, mouth bearded. Ligule 0.2$0.4 \mathrm{~mm}$ long, a ciliate rim with narrow membrane at base. Leaf blades $7-12 \times 0.3-0.5 \mathrm{~mm}$, linear to lanceolate, upper surface scabrid. Panicles 4-15 $\times 4-6$ $\mathrm{cm}$, lax to effuse; branches $6-10 \mathrm{~cm}$ long, alternate, near capillary; axils glabrous; pedicels $2-5 \mathrm{~mm}$ long. Spikelets 3-5 × 0.8-1.1 mm, 5-20-flowered, lanceolate to elliptic, dull green to greyish; rachilla visible between florets, slender, zigzag; florets loosely arranged on rachilla, disarticulating from below upwards. Glumes linear to lanceolate, chartaceous, 1-nerved, 1-keeled, 
scabrid along keel towards apex, apex acute; lower glume $c .0 .8 \times 0.2 \mathrm{~mm}$; upper glume $c .1 \times 0.4 \mathrm{~mm}$. Lemmas $1.2-1.3 \times$ c. $0.5 \mathrm{~mm}$, ovate, chartaceous, 3 nerved, narrowly 1-keeled, glabrous along keel, apex subacute to submucronate. Paleas $0.8-1 \times 0.4-0.5 \mathrm{~mm}$, persistent or subpersistent, elliptic, 2-nerved, 2-keeled, scaberulous along keels, apex acute; palea flaps almost equal in width to its body. Anthers 2 , up to $3 \mathrm{~mm}$ long, purplish. Caryopses up to $0.4 \times 0.2 \mathrm{~mm}$, ovoid to ellipsoid, deep brown.

Flowering and fruiting: Almost throughout the year.

Distribution: INDIA: Almost throughout; TROPICAL ASIA and AFRICA

Habitat: It prefers moist soils, grows along paddy fields, river beds, stream margins, in wetlands, dry river beds, and around stagnated water, between 10-100 m elevation.

Chromosome nos.: $2 \mathrm{n}=80$ (Tateoka, 1965), $2 \mathrm{n}=20$ (Dujardin, 1979).

Notes: This species is often confused with E. atrovirens and E. nutans, but distinct from them by having slender culms with effuse panicle, smaller spikelets, distantly arranged florets on rachilla and ovoid to ellipsoid caryopses (Vivek \& al., 2013e, 2015c).

Specimens examined: INDIA. Andhra Pradesh: Chittoor District: Chandragiri, 23.02.1914, s.coll. 9997 $(\mathrm{MH})$; East Godavari District: Forest nearby Anigiri Village, 200 m, 25.09.1980, G.V. Subbarao 68552 (MH); Korukonda, 25.05.1959, R.S. Rao 18579 (CAL); Karimnagar District: Aklaspur R.F., 200 m, 30.12.1964, G.V. Subbarao 22511 (CAL, MH); Kurnool District: Chenchukunda Pond, Digwametha, Nallamalais, 325 m, 18.11.1969, J.L. Ellis 32513 (CAL, MH); Medak District: Pegarikutta, on the way to Narsapur, $713 \mathrm{~m}$, 23.09.1958, K.M. Sebastine 6655 (MH); Visakhapatnam District: Mandasa Mandu, 15.10.1930, V. Narayanaswami 4622, 4624 (MH); West Godavari District: Palagudam, 08.10.1930, V. Narayanaswami 4479 (MH); Gopanapalam, Sea level, 25.01.1958, K. Subramanyam 5159 (MH). Daman: Without precise locality, 25.09.1963, M.Y. Ansari 93645 (CAL). Jharkhand: Baidyanath, 10.10.1894, J.D. Hooker 1262 (CAL). Kerala: Kasaragod District: Cheruvathur, 125 m, 26.09.1982, R. Ansari 74308 (MH); Kasaragod, 02.10.1982, R. Ansari 74440 (CAL, MH); Nileshwar, 07.12.1919, s.coll. 16307 (MH); Nileshwar, 2nd station, 07.11.1917, s.coll. 15297 (MH); Pilikode, near Nileshwar, 04.12.1919, s.coll. 16277 (MH); Palakkad
District: Without precise locality, 10.03.1965, K.M. Sebastine 22855 (CAL); Chindakki Forest, 12.01.1980, N.C. Nair 65419 (CAL); Water tank hillock, 150 m, 30.05.1964, E. Vajravelu $19126 \quad(\mathrm{MH})$; Thiruvananthapuram District: Veli, 75 m, M. Mohanan $58553(\mathrm{MH})$. Madhya Pradesh: Bilaspur District: Bilaspur, 30.04.1964, C.M. Arora 3785; Ibid., 26.10.1970, G. Panigrahi 12939 (CAL); Ibid., 29.10.1970, G. Panigrahi 13050 (CAL); Bilaspur, Pratabpur, 08.05.1964, C.M. A rora 3920 (CAL); Damoh District: Without precise locality, 14.02.1979, B.K. Shukla 29789 (CAL); Ibid., 22.02.1979, B.K. Shukla 29844 (CAL); Hosangabad District: Bori, $1000 \mathrm{~m}$, 06.10.1960, J. Joseph 11298 (CAL \& MH); Ibid., 28.12.1962, G. Panigrahi 6464 (CAL); Saugor District: Bina river, Rahatgarh, $475 \mathrm{~m}, 04.11 .1960$, N.P. Balakrishnan 11481 (MH); Ghandigram, 22.12.1962, G. Panigrahi 5593 (CAL); Gwalior, s.dat., C. Maries 52 (CAL); Khargaon, 09.02.1987, M. Prasad 39447 (CAL); Mohli R.F., 500 m, 08.11.1960, N.P. Balakrishnan 11541 (CAL \& MH). Maharashtra: Nagpur District: Bhide Tank, 250 m, 13.11.1957, K. Subramanyam 4551 (MH); Pune District: Lonavla, Bhoma Hills, 01.11.1956, S.K.J. 8937 (CAL). Odisha: Balasore District: Balasur, 13.10.1884, C.B. Clarke 36887 (CAL); Bargarh District: Borasambar, 29.10.1959, G. Panigrahi 20624 (CAL); Sundargarh District: Bhutura, 24.11.1987, D. Namhata 2672 (CAL). Rajasthan: Banswara District: Without precise locality, 22.10.1976, V. Singh 3788 (CAL); Jhalawar District: Dug Kyasra, 16.12.1964, D.M. Verma 6777 (CAL). Tamil Nadu: Chenglapatu District: On the way to prehistoric caves of Gudium, c. 50 m, 24.01.1992, Azhakanandam, Krishnan, Ravichandran \& Samson 235 (MH); Kancheepuram District: Vandalur, 07.02.1915, s.coll. 11477, 11478 (MH); Ramanathapuram District: Valantharavai, $50 \mathrm{~m}$, 07.02.1987, V. Balasubramanyam 1178 (CAL, MH); Ibid., 23.02.1988, V. Balasubramanyam 1645 (MH); Tirunelveli District: Mundanthorai, 16.05.1901, C.A. Barber 2806 (MH). Uttar Pradesh: Champaran District: Without precise locality, 26.11.1907, I.H. Burkill 29362 (CAL); Mirzapur District: Mirzapur, 27.10.1964, G. Panigrahi 2090 (CAL); Ibid., 07.03.1970, G. Panigrahi 8499 (CAL); Saharanpur District: Saharanpur, Gangetic Plain, 13.11.1886, J.F. Duthie 6589 (CAL). West Bengal: Darjeeling District: Kalimpong, Eastern Himalaya, s.d., S.K. Mukerjee 5077 (CAL); Hooghly District: Hooghly, 11.09.1968, Subire Sen 753 (CAL); Howrah District: Without precise locality, Oct. 1894, J.D. Hooker 1311; Ibid., 19.09.1894, J.D. Hooker 1314; Ibid., 26.10.1894, 


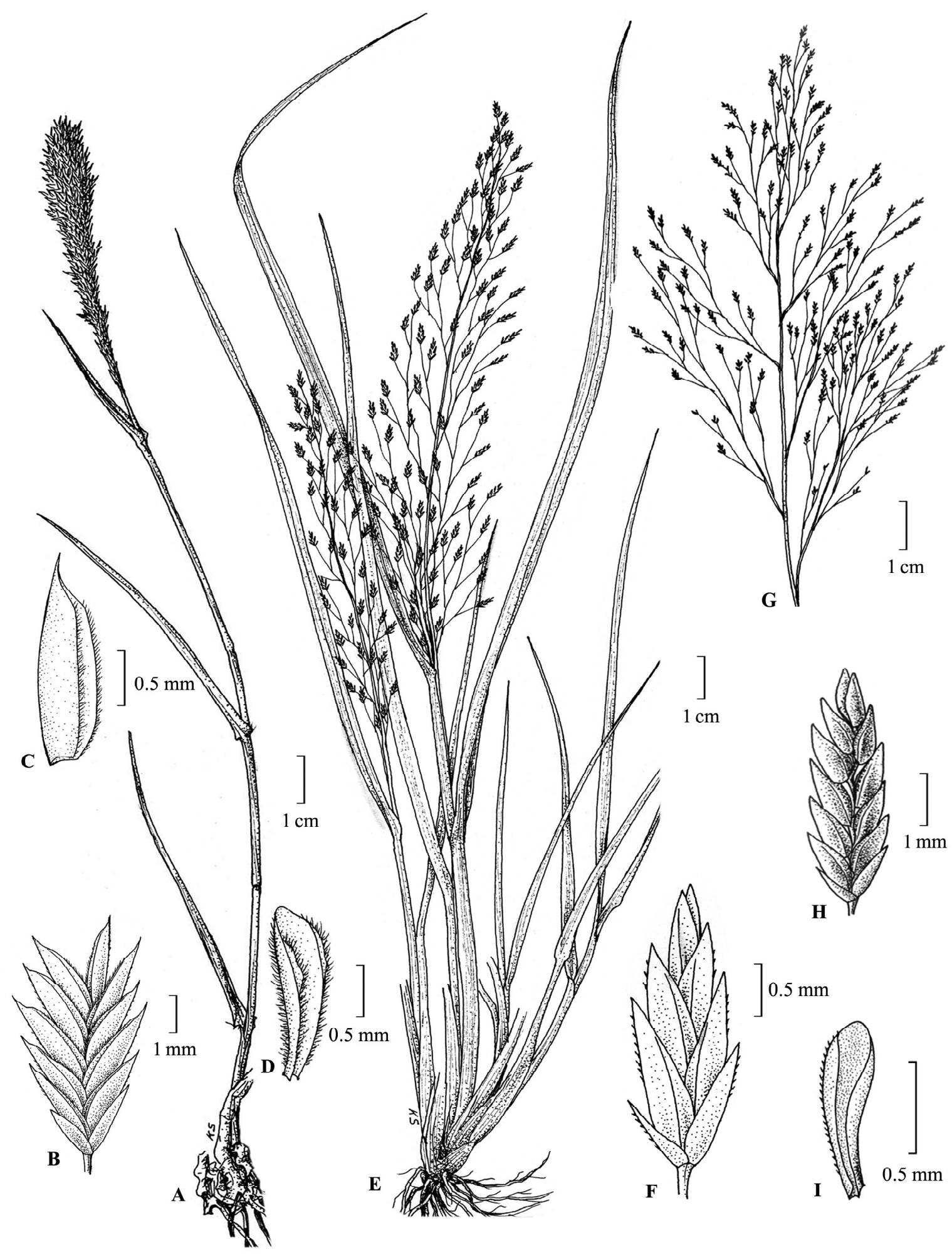

Fig. 4. Eragrostis deccanensis Bor (A-D). A. Habit; B. Spikelet; C. Lemma-lateral view; D. Palea-lateral view. E. ferruginea (Thunb.) P. Beauv. (E-F). E. Habit; F. Spikelet. E. gangetica (Roxb.) Steud. (G-I). G. Inflorescence; H. Spikelet; I. Palea-ventral view. 
J.D. Hooker 1307; Ibid., January 1895, Mokim 1494 (CAL); Horinhpur, 18.10.1964, S.S.R. Bennet 1031, 1032 (CAL); Indian Botanic Garden, 10.10.1968, D.K. Banerjee 21616 (CAL); Malda District: Without precise locality, 29.10.1959, G. Panigrahi 20591 (CAL); Ibid., 01.11.1959, G. Panigrahi 20624 (CAL); Ibid., 14.12.1980, P.C. Nanda 2364 (CAL); Purulia District: Without precise locality, s.d., K.C. Malik 993 (CAL).

15. Eragrostis henryi Vivek, G.V.S. Murthy \& V.J. Nair in Nelumbo 54: 9. 2012. Type: INDIA, Tamil Nadu, Kanyakumari district (Near Anjugramam), 25 m, 27.03.1979, A.N. Henry 6156 (holo CAL!, iso MH!).

(Fig. 5 \& Plate 2)

Annual. Culms 5-20 cm high, geniculate; nodes brownish; internodes $c .3 \mathrm{~cm}$ long, with glandular ring close below nodes. Leaf sheaths $c .2 \mathrm{~cm}$ long, glandular on nerves, ciliate along one margin or glabrous, mouth bearded. Ligule a fringe of cilia. Leaf blades $1-3 \mathrm{~cm}$ long, linear to lanceolate, margins with raised glands, upper surface glabrous, lower surface with glandular dots along midrib. Panicles $3-7 \times 1.5-4 \mathrm{~cm}$, ovate to oblong, with non-viscous glandular band or ring on peduncle close below panicle; primary panicle branches up to $3 \mathrm{~cm}$ long, alternate, stiff, with crateriform glands; axils glabrous; pedicels $1-1.5 \mathrm{~mm}$ long, stiff, with crateriform glands. Spikelets $8-25 \times 1.5-2 \mathrm{~mm}$, up to 55-flowered, 1-9 per branch, linear, narrowly curved, pinkish to yellowish; rachilla slender, zigzag; florets closely arranged on rachilla, disarticulating from below upwards. Glumes ovate to lanceolate, chartaceous, 1nerved, 1-keeled, scabrous along keel, apex acute; lower glume 0.8-1 mm long; upper glume 0.9-1.2 mm long. Lemmas $1-1.3 \times$ c. $0.7 \mathrm{~mm}$, elliptic to narrowly ovate, chartaceous, 3-nerved, 1-keeled, scabrous along keel, apex acute, obtuse when spread. Paleas $c .1 \mathrm{~mm}$ long, persistent, elliptic to oblanceolate, 2-nerved, 2-keeled, scaberulous along keels above middle, apex acute to obtuse; palea flaps narrower than its body. Anthers 3, up to $0.3 \mathrm{~mm}$ long. Caryopses $0.5-0.6 \times c .0 .3 \mathrm{~mm}$, ovoid, apex acute to obtuse, base truncate, laterally flattened to narrowly grooved, yellowish brown.

Flowering and fruiting: March.

Distribution: INDIA: Tamil Nadu. Endemic.

Habitat: Occurs in sandy soil, c. $25 \mathrm{~m}$.

Notes: Long, linear and narrowly curved spikelets of $E$. henryi closely resemble those of E. tremula. However, the latter species differs from E. henryi in many characters including the large dimension of panicle, capillary and flexuous pedicels and absence of glands (Vivek \& al., 2012).

Specimens examined: INDIA. Tamil Nadu: Kanyakumari District: Anjugramam near area, 27.03.1979, A.N. Henry 61563 (CAL, MH).

16. Eragrostis jainii Vivek, G.V.S. Murty \& V.J. Nair in Nelumbo 55: 1. 2013. Type: INDIA, Kerala state, Kannur District, Manjeshwar, 100 m, 13 October 1979, R. Ansari 64888 (holo CAL!, iso MH!).

(Fig. 5 \& Plate 2)

Annual. Culms 20-60 cm high, erect to decumbent; nodes brownish; internodes $2-8 \mathrm{~cm}$ long, glabrous. Leaf sheaths $c .3 \mathrm{~cm}$ long, mouth bearded. Ligule a ciliate rim with narrow membranous base. Leaves basal; blades $2-9 \times 0.2-0.5 \mathrm{~cm}$, linear to involute, stiff and attenuate to a point, upper surface villous-hairy. Panicles 4-23 $\times$ 1-2 cm, narrow, linear; branches alternate, appressed, effuse, angled, scabrous along angles; pedicels 1-5 mm long, scabrous along angles. Spikelets 5-10 × 2-3.5 $\mathrm{mm}, 6-80$-flowered, ovate to lanceolate or elliptic, apex sharply acute, purplish; rachilla persistent, thick, terete or wavy to almost straight; florets firmly arranged on rachilla, disarticulating from below upwards. Glumes unequal, lanceolate, chartaceous, margins with or without minute hairs at base, 1-nerved, 1-keeled, scabrous along keel, apex acuminate to subacuminate; lower glume $1-1.5 \times 0.2-0.25 \mathrm{~mm}$; upper glume $1.5-2$ $\times 0.3-0.4 \mathrm{~mm}$. Lemmas $1.7-2 \times 0.7-1 \mathrm{~mm}$, ovate to lanceolate, chartaceous, dorsal side nearly globose at base, margins with or without minute cilia at base, 3nerved, 1-keeled towards apex, more or less rounded at base, apex acuminate. Paleas 1.7-2 $\times 0.4-0.8 \mathrm{~mm}$, caducous, elliptic to lanceolate, curved on back, 2nerved, nerves reach only up to $3 / 4$ of overall length of palea, 2-keeled, ciliate along keels, apex with up to 0.5 $\mathrm{mm}$ long acuminate projection, at times split opened into 3-minute lobes; palea flaps narrower than its body. Anthers 2, c. $0.3 \mathrm{~mm}$ long, purplish. Caryopses 0.4-0.5 $\times 0.25-0.35 \mathrm{~mm}$, obovoid, reddish brown.

Flowering and fruiting: October-December.

Distribution: INDIA: Karnataka and Kerala. Endemic.

Habitat: Along sandy beaches, between 50 and $100 \mathrm{~m}$.

Notes: This species resembles E. unioloides but differs by its appressed panicle branches, sharply acute spikelets, acuminate lemmas and ciliate paleas. Another diagnostic character of palea of E. jainii is the nerves on keels of palea that extent only up to $3 / 4$ of the overall length of the palea and the apical portion remains as an 
acuminate projection (Vivek \& al., 2013c).

Specimens examined: INDIA. Karnataka: Mysore, Paygambalam Beach, 17.11.1972, T.A. Rao 10022 (CAL); Uttara Kannada District: North Kanara, Sea Coast, 10.12.1883, W.A. Talbot 869 (CAL); Barwar, 27.08.1885, W.A. Talbot 1296 (CAL). Kerala: Alappuzha District: Alappuzha Beach, 03.11.1972, T.A. Rao 9717 (CAL); Kannur District: Manjeshwar, 13.10.1979, R. Ansari 64888 (CAL); Muzhappilangad, 16.12.1979, V.S. Ramachandran 65258 (CAL).

17. Eragrostis japonica (Thunb.) Trin. in Mem. Acad. Imp. Sci. St.-Petersbourg, Ser. 6, Sci. Math. 1: 405.1830.

Poa japonica Thunb. in Murray, Syst. Veg., ed. 14: 114. 1784. Diandrochloa japonica (Thunb.) A.N. Henry in Bull. Bot. Surv. India 9: 290. 1968. Type: JAPAN, Herb. Thunberg 2252 (holo UPS, frag. in BRI, photo K).

Eragrostis interrupta sensu Stapf in Hook.f., Fl. Brit. India 7: 316. 1896, incl. vars., non (R. Br.) P. Beauv. 1812.

Poa diarrhena Schult. \& Schult.f., Mant. 3: 616. 1824. Eragrostis diarrhena (Schult. \& Schult.f.) Steud., Syn. Pl. Glumac. 1: 266. 1854. Diandrochloa diarrhena (Schult. \& Schult.f.) A.N. Henry in Bull. Bot. Surv. India 9: 290. 1968. Type: Roxburgh s.n. (holo BM, iso CAL!, K [K000245081 image!], frag. in BRI).

Eragrostis diplachnoides Steud., Syn. Pl. Glumac. 1: 268. 1854. Type: AFRICA, Sudan, Nubia, 16 March 1840, Kotschy 346 (holo HAL0107094, image!, UPS; iso K, L, n.v.).

(Fig. 5 \& Plate 4)

Annual or Perennial. Culms 30-200 cm high, erect, at times geniculate. Leaf sheaths keeled towards apex. Ligule 0.3-0.4 mm long, membranous. Leaf blades 12$34 \times 0.4-0.7 \mathrm{~cm}$, linear to lanceolate, base rounded to shallowly cordate, margins flat to inrolled. Panicles 8$65 \times 1-4 \mathrm{~cm}$, open, narrowly contracted, lax or interrupt; primary panicle branches alternate or subverticillate; pedicels terete to flat. Spikelets 1.5-3.4 $\times 0.5-0.9 \mathrm{~mm}, 3-13$-flowered, oblong to lanceolate, greenish yellowish; rachilla fragile, zigzag; florets loosely arranged on rachilla, disarticulating from above downwards. Glumes ovate to lanceolate or oblonglanceolate, chartaceous or hyaline, apex acute to acuminate, 1-nerved, 1-keeled, scabrous on keel; lower glume $0.4-0.8 \times 0.3-0.4 \mathrm{~mm}$; upper glume $0.6-1 \times 0.4-$ $0.5 \mathrm{~mm}$. Lemmas $0.8-1.2 \times c .0 .4 \mathrm{~mm}$, ovate to lanceolate, chartaceous, hyaline, 3-nerved, lateral nerves straight, 1-keeled or keel absent, scabrous on keel, apex acute to acuminate or narrowly emarginate.
Paleas $0.5-0.8 \times 0.3-0.4 \mathrm{~mm}$, caducous, ovate to elliptic, chartaceous or hyaline, 2 -nerved, nerves reach only up to $3 / 4$ of overall length of palea, 2 -keeled, scaberulous or glabrous on keels, apex incise into 3-lobes; palea flaps narrower than its body. Anthers 2, 0.2-0.4 mm long, yellowish to purplish. Caryopses $0.3-0.5 \times c .0 .2 \mathrm{~mm}$, obovoid, brownish.

Flowering and fruiting: Almost throughout the year.

Distribution: INDIA: Almost throughout; SE ASIA, AFRICA and AUSTRALIA.

Habitat: Grows on damp soils near water-logged places, moist riverbeds, and rock crevices during rain, around large perennial tanks, in forests, up to $1000 \mathrm{~m}$.

Chromosome no.: 2n = 20 (Larsen, 1963).

Vernacular names: Chiksi, Dhuria, Ghodila, Ghorila, Madra, Pohe, Poche, Shetpatra (Bengali); Kadu gasagasai hullu, Pini hullu (Kannada); Kanjira pullu (Tamil).

Notes: Bor (1960) treated E. diarrhena, E. diplachnoides and E. japonica as three distinct species. Cope (1982) merged E. diarrhena and E. diplachnoides under E. japonica, and treated them as three different forms of E. japonica. While combining these species Cope (l.c.) says that he is reducing these species to synonymy eventhough this may seem unsatisfactory. A careful scruitiny of the characters cited under these groups and the detailed studies made during the present investigation showed that these three forms are markedly different in the inflorescence dimension and branching. Primary panicle branches of $E$. diplachnoides are capillary, longer, loose, and solitary. In E. diarrhena and E. japonica branches are shorter and solitary with much clustered spikelets in $E$. diarrhena, but with 3 or 4 whorls in E. japonica. The polished appearance of the panicle of E. japonica is another diagnostic feature which is absent in other two forms. However, it is studied in the specimens of the three forms that the charatcres of spikelets and its components are overlapping in many cases. Therefore, we follow Cope (l.c.) in the placement of E. diarrhena and E. diplachnoides as synonym of E. japonica.

E. japonica attaining a height of about 2 meter, is probably the tallest among Indian Eragrostis. Presence of a truly membranous ligule (up to $0.5 \mathrm{~mm}$ long) and 3-lobed palea are the unique characters of this species.

Specimens examined: INDIA. Andhra Pradesh: Ananthapuramu District: Garugudukona, 21.12.1982, 
T. Pullaiah 957 (MH); East Godavari District: Dhowleswaram, 01.05.1902, C.A. Barber 4352 (MH); Chittoor District: Balapalle, 250 m, 22.02.1963, J.L. Ellis 15754 (MH); Kurnool District: 12.12.1963, J.L. Ellis 18074 (CAL); Khammam District: Badrachalam Forest, \pm 250 m, 23.02.1994, R. Chandrasekaran $99033(\mathrm{MH})$; Ratham Hutta Hills, $200 \mathrm{~m}, 25.02 .1994, R$. Chandrasekaran 99059 (MH); Tekkulagudem, 06.04.1988, N. Ramarao \& T. Ravisankar 86105 (MH); Krishna District: Kondapalle Fort, 19.02.1986, P. Venkanna 6025 (MH); Kurnool District: 19.11.1969, J.L. Ellis 32547 (CAL); Nellore District: Duttalur, March 1918, C.E.C. Fischer 4306 (FRC); Ramayapatnam, 26.01.1929, K. Cheriyan Jacob 18361 (CAL); West Godavari District: Vadapalli, Polavaram Agency, 08.03.1963, D.C.S. Raju 142 (CAL). Bihar: Champaran District: Udaipur Forest, 10.11.1963, B.V. Shetty 171 (CAL); Gaya District: Chota Nagpur to Hazaribagh, Oct. 1873, C.B. Clarke 21149 (CAL); Chotowlea, 02.11.68, S. Kurz s.n., CAL Acc. No. 541556 (CAL); Manda, Hazaribagh, 27.11.1874, C.B. Clarke 25004 (CAL); Patna District: Ghalawar, 27.12.1965, R.B. Mazumdar 10045 (CAL); Parasnath District: Chota Nagpore, 05.12.1814, C.B. Clarke 25158 (CAL). Chhattisgarh: Bastar District: Keskal Plantations, 600 m, 18.11.1958, K. Subramanyam 7174 (MH). Gujarat: Vallabh Vidyanagar District: 18.02.1967, s.coll. 2706 (CAL). Jharkhand: Sahibganj District: Rajmahal, Oct. 1894, Mokim 1471 (CAL). Karnataka: Bellary District: May 1886, J.S. Gamble 17765 (CAL); Chickmangalur District: Kadur, Khed Taluka, 29.01.1961, K.P. Janardhanan 69038 (CAL); Coorg District: Fraserpet, 1955, A.D. Fraserpet s.n., MH Acc. No. 97090 (MH). Kerala: Kannur District: Thirunelli, $\pm 800 \mathrm{~m}$, 08.05.1979, V.S. Ramachandran 62719 (MH); Vadakkanchery, 27.12.1919, s.coll. 16332 (MH); Kollam District: Shendurney river valley, Vandalodu, 01.06.1964, K.N. Subramanyan 1511 (FRC); Palakkad District: Thannimoodu, Mukkali, 550 m, 27.01.1980, P. Bhargavan 65724 (CAL \& MH); Walayar, 06.01.1911, C.E.C. Fischer 2447 (FRC); Walayar, Teak Reserch Plantation plot, 22.12.1973, K.N. Subramanyan 4685 (FRC); Thrissur District: Chalakkudi, 300 m, K. Ramamurthy 66289 (MH); Chalakkudi riverside, Kodasseri, 300 m, 22.03.1980, K. Ramamurthy 66289 (MH). Madhya Pradesh: Bilaspur District: Khondra, Canrajpohar, 23.10.1970, G. Panigrahi 12787 (CAL); Damoh District: 23.01.1978, P.C. Pant 26884 (CAL); Singhgergarth, 23.04.1980, B.K. Shukla 30149 (CAL); Jubbalpore District: Heran River side, 470 m, 14.03.1962, K.M. Sebastine 13960
(MH); Khargaon District: Barodi, 15.11.2000, Bipin Kumar Sinha 530509 (BSA); Narmada Valley, Narmada Sagar, 07.05.1986, B.K. Shukla 37759 (BSA); Pepal Jhopa. 06.02.1987, M. Prasad 39355 (CAL); Khawwa District: 13.01.1889, J.F. Duthie 8503 (CAL); Mandla District: Bichhia, 07.02.1961, J. Joseph 12215 (CAL \& MH); Khari R.F., 675 m, 26.01.1961, J. Joseph $13415(\mathrm{MH})$; Narmada river bed, $500 \mathrm{~m}, 03.12 .1961, J$. Joseph 13528 (CAL \& MH); Rewa District: 11.02.1959, K.M. Sebastine 7711 (CAL); Raigarh District: Pithora, Deori, 14.10.1976, D.M. Verma 25707 (BSA); Silot block, Ghargude Range, 09.11.1971, G. Sengupta 16062 (BSA). Satna District: Majhgawan, 23.02.1987, $R$. Prasad 38455 (CAL); Shivpuri District: Shivpuri National Park, 18.07.1972, G. Panigrahi 17041 (BSA); Sidhi District: Majhauli, on the road to Durbi, 19.01.1971, G. Sengupta 14124 (BSA); Seoni District: Umadini, 23.12.1975, L.K. Banerji \& V.J. Nair 23245 (BSA). Maharashtra: Khandesh District: 05.01.1957, S.K. Jain 11009 (CAL); Nagpur District: Gourkilla, Kalahandi, 07.05.1956, S.K. Mukerjee 4298 (CAL); Nashik District: Adgaon, 28.11.1961, K.P. Janardhanan 75965 (CAL); Parbhani District: Bori, 23.12.1962, G. Panigrahi 6375 (CAL); Sindhudurg District: Malavani. 13.12.1956, S.C. Agarwal 1063 (CAL); Thana District: Batana, 13.01.1903, G.A. Rayan 175 (BSI). Odisha: Gajapathi District: Chamundia, Mahanadi, 20.03.1943, K. Biswas 6249 (CAL); Ganjam District: Gulley, 121 m, January 1884, J.S. Gamble 13687 (MH). Rajasthan: Barmer District: Devalai Village, 16.10.1975, B.V. Shetty 2197 (CAL); Banswara District: Bagaycha Forest, 27.03.1977, V. Singh 4279 (CAL); Bikaner District: 26.09.1975, G.P. Roy 2165 (CAL); 28.11.1975, G.P. Roy 2377 (CAL); Kolayat, 28.11.1975, G.P. Roy 2361 (CAL); Ganganagar District: Khanuwala, 05.10.1977, G.P. Roy 4899 (CAL); Satrana Village, 11.11.1976, G.P. Roy 3824 (CAL); Jalor District: Luni Riverbank, 17.01.1978, B.L. Vyas 5294 (CAL); Pali District: Jawai Dam, 11.11.1974, B.V. Shetty 1434 (CAL); Sarparnia, 28.12.1985, R.P. Pandey 19434 (CAL). Tamil nadu: Coimbatore District: Agri. College, 468 m, 21.11.1965, M. Chandrabose 28838 \& 28841 (MH); Coimbatore, Aug. 1902, C.A. Barber 4884 (MH); Kollegal, 14.02.1930, V. Narayanaswami 19987 (MH); Siruvani, Pungamcheri R.F, \pm 457 m, 26.01.1932, M. Ramavelu 64 (MH); Velliangiri Hills, 667 m, 18.02.1957, K.M. Sebastine 2376 (CAL \& MH); Walayar Dam Area, 04.09.2010, C.P. Vivek 126139 (MH); Thadagai Hills, Andiyur, 11.07.2010, C.P. Vivek $126132(\mathrm{MH})$; Dharmapuri District: Hogainakal, 80 m, 11.02.1927, K.C. Jacob 17989 (MH); Madurai District: Kumbukere, 
400 m, 15.06.1961, K.M. Sebastine 12597 (MH); Sirimalai, 266 m, 18.02.1958, K. Subramanyam 5369 (MH); Ramanathapuram District: Gopalapuram burial ground, Pduppettai, $\pm 15 \mathrm{~m}, 15.02 .1979$, N.C. Nair 60813 (MH); Karaikudi, 100 m, 28.11.1977, N.C. Nair 51755 (CAL \& MH); Karisalkulam, \pm 50 m, 23.12.1989, V. Balasubramanyam 2300 (MH); Krishnathevar Thoppu, \pm 225 m, 09.03.1980, S.R. Srinivasan 63633 $(\mathrm{MH})$; Melakkadu Forest, Sivaganga, $100 \mathrm{~m}$, 17.12.1964, K. Ramamurthy 22744 (CAL \& MH); Periyar River, 275 m, 16.02.1979, N.C. Nair 60860 (MH); Sreevilliputtur, Kansapuram Beat Riverside, 210 m, 18.07.1965, E. Vajravelu 24776 (CAL \& MH); Uttarakosamangai, $\pm 75 \mathrm{~m}, 08.02 .1987, \quad V$. Balasubramanyam 1233 (MH); Salem District: Gandiyan lake, 460 m, E. Vajravelu 22454 (MH); Thanjavur District: Mannai, 05.03.1987, S. Ragupathi 291 (MH); Nannilam to Aduthurai, 50 m, 28.01.1978, K. Ramamurthy 53641 (MH); Pattukottai to Tanjore, $50 \mathrm{~m}, 01.02 .1978$, K. Ramamurthy 53718 (MH); Velanganni, along the side of irrigation cannal, $50 \mathrm{~m}$, 21.05.1978, V.J. Nair 57031 (MH); Tirunelveli District: Ambasamudram, 13.05.1901, C.A. Barber 2731 (MH); Mundanthorai, 16.05.1901, C.A. Barber 2833 (MH); Vadugapatty, 08.02.2011, C.P. Vivek \& C. Kalidass 126149 (MH); Virudhunagar District: Tiruchuli, 11.03.1953, C.H. Maduram 21876 (MH). Uttar Pradesh: Allahabad District: Dargang, 10.11.1964, T. Rajagopal 6271 (BSA); Bagia Naini, 24.12.1963, V.N. Singh 2216 (BSA); Naini, 28.12.63, V.N. Singh 2216 (CAL); Bahraich District: Abdullagunj, Chardharange, 18.11.1964, G. Panigrahi 6421 (BSA); Motipur, Kheri, 22.11.1964, G. Panigrahi \& O.P. Misra 6587 (BSA); Banda District: Chitrakut, s.dat., C.M. Arora 2604 (CAL); Gorakhpur District: Baran West Lehri, 02.11.1963, C.M. Arora 1467 (BSA); Mirsapur District: Dhandhraul Dam site, 20.12.1970, G. Panigrahi 13445 (BSA); Gautam Buddha Nagar District: Dadri Dam site, 06.03.1970, G. Panigrahi 8436 (CAL); Khaurwa District: Khari, 13.12.1888, J.F. Duthie 8505 (CAL); Saharanpur District: Saharanpur, Oct. 1888, J.F. Duthie 7708 (CAL); Shahjahanpur District: Indalpur, Shahjanpur, 11.10.1885, J.F. Duthie 5106 (CAL); Varanasi District: Latifshah, 15.05.1968, G. Panigrahi 11831 (BSA). West Bengal: Bankura District: Thitimiti, 10.10.1982, M.N. Sanyal 1471 (CAL); Burdwan District: Bhedia, 18.03.1965, A.K. Dutt 686 (CAL); Burakur, 27.01.1892, C.B. Clarke 25249 (CAL); Eden Canal, 16.10.1894, J.D. Hooker 1222 (CAL); Darjeeling District: Bambuka Bosthi, Kalimpong, 06.11.1960, K. Thothathri 9417 (CAL); Hooghly District: without precise locality, 08.12.1967, Subire Sen 207 (CAL); Uttarpara, Hooghly, 07.08. 1968, Subire Sen 621 (CAL); Jalpaiguri District: Moyasabari, Bhutanghat, 05.12.1975, J.K. Sikdar 4143 (CAL); Kolkata District: Without precise locality, 1836, Joh. W. Helfer 298 (CAL).

18. Eragrostis lehmanniana Nees, Fl. Afr. Austral.: 402. 1841. Type: SOUTH AFRICA., Cabo de Buena Esperanza, J.F. Drege s.n. (iso BM, LE).

Eragrostis chaunantha Pilg. in, Bot. Jahrb. Syst. 40: 84. 1907. Eragrostis lehmanniana Nees var. chaunantha (Pilg.) De Winter in Meredith, Grass. Pastures South Africa: 147. 1955. Type: BOTSWANA, Kalahari, Dec. 1904, L. Schultze 290 (holo B [B100272791, image!], iso K [K000365814, image!]).

(Fig. 5 \& Plate 2)

Perennial. Culms (20-) 40-80 cm high, erect to geniculate, sometimes rooting at lower nodes; nodes brownish; internodes $c .7 \mathrm{~cm}$ long. Leaf sheaths $4-8$ $\mathrm{cm}$ long, margins finely ciliate, sometimes shortly pilose at base. Ligule $0.3-0.7 \mathrm{~mm}$ long, a fringe of cilia. Leaf blades $2-15 \times 0.1-0.3 \mathrm{~cm}$, flat, margins infolded at maturity, upper surface scaberulous. Panicles $7-18 \times$ 2-8 cm long, more or less open or oblong; branches 1$8 \mathrm{~cm}$ long, alternate to subwhorled, appressed to main axis; axils glabrous. Spikelets 5-12 (-14) $\times 0.8-1.2 \mathrm{~mm}$, 4-12 (-14)-flowered, linear to lanceolate or oblong, light green to greyish; rachilla zigzag; florets more or less closely arranged on rachilla, disarticulating from below upwards. Glumes oblong to lanceolate, chartaceous, margins mostly entire, at times finely serrulate, 1-nerved, 1-keeled, apex acute; lower glume 1-1.5 mm long; upper glume 1.3-2 mm long. Lemmas $1.5-1.7 \times 0.6-0.7 \mathrm{~mm}$, ovate to elliptic, membranous, 3-nerved, lateral nerves obscure, 1-keeled, apex obtuse to subacute. Paleas 1.4-1.7 $\times$ c. $0.45 \mathrm{~mm}$, persistent, oblanceolate, 2-nerved, 2-keeled, scaberulous along keels above middle, apex obtuse; palea flaps narrower than its body. Anthers 3, 0.6-0.9 mm long, yellowish. Caryopses $0.6-0.8 \times c .0 .3 \mathrm{~mm}$, ellipsoid to ovoid, base narrowly truncate, dorsally flattened to grooved, yellowish brown.

Distribution: Introduced in INDIA (Bor, 1960; Moulik, 1997); SOUTH-WEST AFRICA.

Chromosome nos.: $2 \mathrm{n}=40$ (Pienaar, 1955), $2 \mathrm{n}=60$ (Gould, 1960; Halvorsen \& Guertin, 2003).

Specimens examined: NORTH AMERICA. Arizona: without precise locality, 21.05.1966, L.H. Harvey \& J.C. Elliott 7293 (CAL). Texas: without precise locality, 


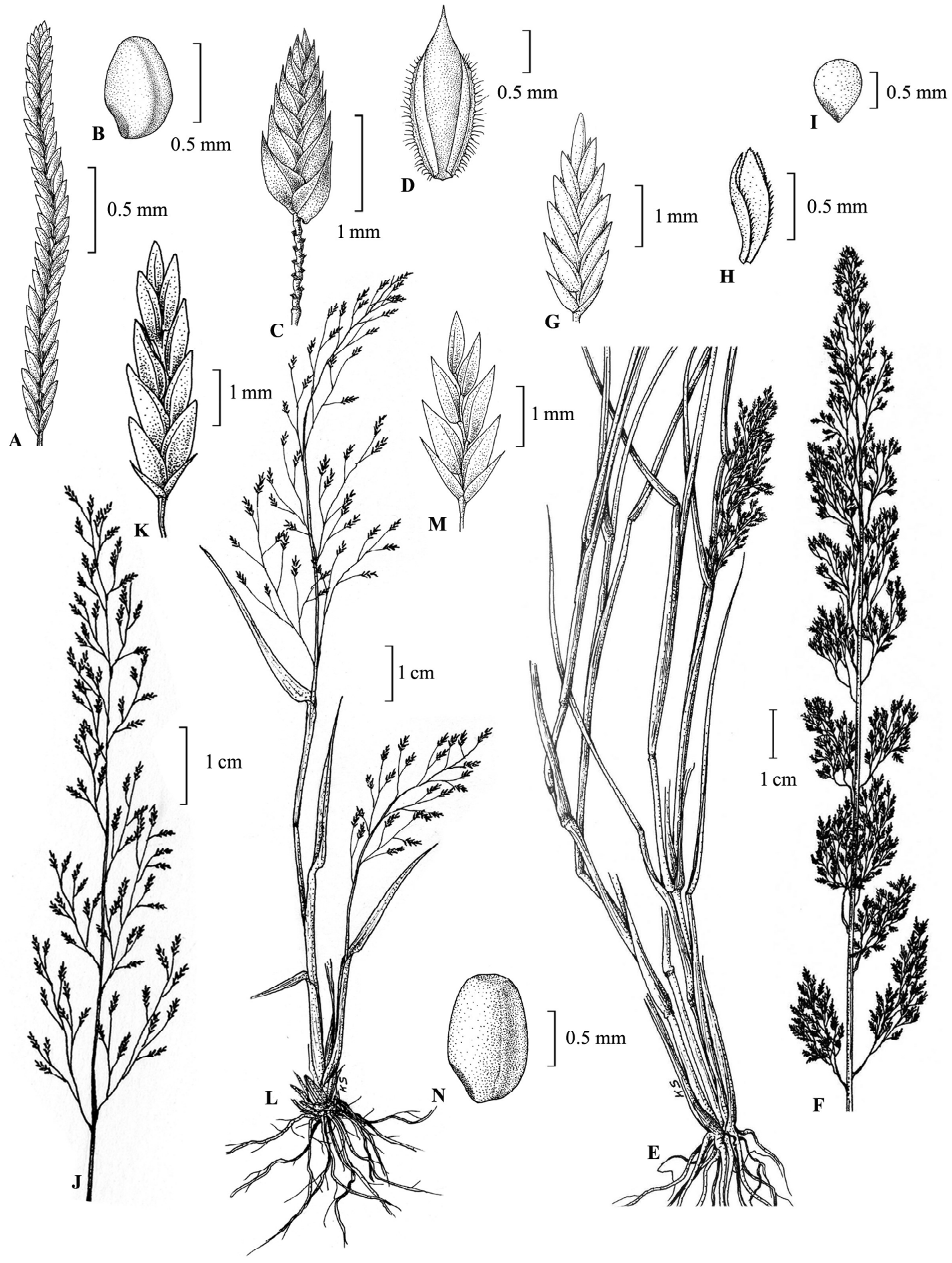

Fig. 5. Eragrostis henryi Vivek, G.V.S. Murthy \& V.J. Nair (A-B). A. Spikelet; B. Caryopsis. E. jainii Vivek, G.V.S. Murty \& V.J. Nair (C-D). C. Spikelet; D. Palea-ventral view. E. japonica (Thunb.) Trin. (E-I). E. Habit; F. Inflorescence; G. Spikelet; H. Paleadorsal view; I. Caryopsis. E. lehmanniana Nees (J-K). J. Inflorescence; K. Spikelet. E. macilenta (A. Rich.) Steud. (L-N). L. Habit; M. Spikelet; N. Caryopsis. 
26.05.1966, L.H. Harvey \& J.C. Elliott 7318 (CAL).

19. Eragrostis macilenta (A. Rich.) Steud., Syn. Pl. Glumac. 1: 268. 1854.

Poa macilenta A. Rich., Tent. Fl. Abyss. 2: 428. 18501851. Type: ETHIOPIA, 17 September 1839, $R$. Quartin-Dillon et Petit s.n. (holo P [P00484394, image!], iso LE, n.v.).

(Fig. 5 \& Plate 2)

Annual. Culms 7-60 cm high, slender, erect or ascending; nodes brownish; internodes $c .4 \mathrm{~cm}$ long. Leaf sheaths $c .2 \mathrm{~cm}$ long, glabrous. Ligule a fringe of cilia. Leaf blades $2-20 \times 0.2-0.5 \mathrm{~cm}$, flat. Panicles 3$35 \mathrm{~cm}$ long, ovate to elliptic, open, effuse; branches alternate, capillary, whorled at lower nodes; pedicels 2-12 mm long, capillary. Spikelets 3-6 × 1-2 mm, 514-flowered, ovate to lanceolate or oblong, light green to almost blackish; rachilla persistent; florets loosely arranged on rachilla, disarticulating from below upwards. Glumes 1.1-1.5 mm long, narrowly ovate to lanceolate, chartaceous, 1-nerved, 1-keeled, glabrous or scabrid along keel towards apex, apex acuminate. Lemmas $1.3-1.6 \times$ c. $0.7 \mathrm{~mm}$, ovate to elliptic or narrowly lanceolate, chartaceous, 3-nerved, 1-keeled, scabrous along keel, apex acute. Paleas $1.2-2 \times 0.4-0.5$ $\mathrm{mm}$, persistent, elliptic to lanceolate, 2-nerved, 2keeled, scaberulous along keels above middle; palea flaps almost equal in width to its body. Anthers 3, 0.3$0.5 \mathrm{~mm}$ long, brownish to purplish. Caryopses $c .0 .5 \times$ $0.3 \mathrm{~mm}$, broadly oblong, both ends truncate, ventrally grooved, dark reddish.

Flowering and fruiting: October-August.

Distribution: INDIA: Andhra Pradesh and Tamil Nadu; EAST AFRICA

Habitat: Grows in drier areas.

Note: Kalidass \& al. (2019) mentioned its occurrence in Andhra Pradesh.

Specimen studied: AFRICA. Without precise locality, 05.09.1902, A. Pappi 2672 (CAL).

20. Eragrostis maderaspatana Bor, Grass. Burma Ceylon India Pakistan: 509. 1960.

Eragrostis willdenowiana Nees [in Wight, Cat. Ind. Pl.: n. 1779] ex Stapf in Hook.f., Fl. Brit. India 7: 322. 1896, non Nees ex Hook. \& Arn., Bot. Beechey Voy.: 252. 1832 \& in Nov. Act. Nat. Cur. 19: Suppl. 1. 205. 1843. Lectotype: INDIA, s.d., Wight s.n.: Herb. Wight propr no. 1779 (K [K000245101, image!]), designated by Vivek \& al. in Nelumbo 57: 40. 2015. (Fig. 6 \& Plate 4)
Annual. Culms 5-46 cm high, geniculate; nodes brownish; internodes $c .6 \mathrm{~cm}$ long, with glandular ring close below nodes. Leaf sheaths $c .3 \mathrm{~cm}$ long, sparsely ciliate along one margin or glabrous, mouth bearded. Ligule a fringe of cilia. Leaf blades $2-13 \times 0.1-0.3 \mathrm{~cm}$, linear to lanceolate, margins glandular or eglandular, upper surface sparsely tubercle based ciliate or glabrous. Panicles 3-15 × 2-4 cm, with non-viscous glandular patches on peduncle close below panicle, open, ovate; branches alternate, more or less glandular, scabrous along angles; axils glabrous; pedicels $1-5 \mathrm{~mm}$ long with or without a glandular patches, scabrous along angles. Spikelets 7.6-5 × 1-1.3 mm, up to 30-flowered, ovate to lanceolate, olive green to grey with or without a purplish tinge; rachilla zigzag; florets closely arranged on rachilla, disarticulating from below upwards. Glumes unequal, ovate to lanceolate, chartaceous, 1nerved, at times nerve obscure, 1-keeled, scabrid along keel, apex acute; lower glume $0.5-0.75 \times 0.25-0.3 \mathrm{~mm}$; upper glume $1-1.27 \times 0.4-0.5 \mathrm{~mm}$. Lemmas $1.5-1.8 \times$ 0.6-0.7 mm, broadly elliptic, chartaceous, 3-nerved, 1keeled, scabrid along keel or glabrous, apex acute. Paleas 1.2-1.6 $\times 0.3-0.4 \mathrm{~mm}$, persistent, elliptic, 2nerved, 2-keeled, scaberulous along keels above middle, apex acute to obtuse; palea flaps narrower than its body. Anthers 3, c. $0.2 \mathrm{~mm}$ long, purplish. Caryopses $0.5-0.8$ $\mathrm{mm}$ long, oblong to ellipsoid, both ends truncate, ventrally flattened to slightly grooved, yellowish brown.

Flowering and fruiting: Almost throughout the year.

Distribution: INDIA: Andhra Pradesh, Karnataka, Kerala and Tamil Nadu; SRI LANKA?.

Habitat: Grows in open places, near streams, in scrub jungles, roadsides and wastelands.

Notes: This species resembles E. minor in having glands along leaf margins, culms, panicle and pedicels and persistent palea. But, E. maderaspatana differs from the latter by the ventrally flattened and truncate caryopses which are yellowish brown in colour.

Eragrostis maderaspatana is probably endemic to the peninsular region of India (Andhra Pradesh, Karnataka, Kerala and Tamil Nadu). There was an earlier record of this species (Glenie C.P. 3944), as mentioned by Stapf (1896) and Bor (1960), collected from Trincomale in Sri Lanka. But Lazarides (in Dassan. Revis. Handb. Fl. Ceylon Vol. no.: p. no. 1994) has not mentioned the occurrence of this species in Sri Lanka thus its occurrence in the country is doubtful. 
Specimens examined: INDIA. Andhra Pradesh: Chittoor District: Kuppam, 24.08.1918, s.coll. 15526 (MH); Guntur District: Narasarapet, 01.09.1902; C.A. Barber 4549 (MH). Karnataka: Bangalore District: 1917, R.D.A, B.246 (MH); Bellary District: Hagavi Jaran, 19.09.1916, s.coll. 13771 (MH); 20.09.1916, s.coll. 13794 (MH); Hampi, 14.10.1919, s.coll. 15999 \& 16000 (MH). Tamil Nadu: Coimbatore District: Coimbatore, August 1902, C.A. Barber, 4877 (MH); Southern Forest Ranger's College, near block no. 8, 18.11.1963, K.N. Subramanyam 899 (FRC); Erode District: Sathyamangalam, \pm 243 m, 26.11.1931, S.R. Raju 6184 $(\mathrm{MH})$; Ramanathapuram District: Uttarakosamangai, \pm 20 m, 01.01.1988, V. Balasubramanyam 1514 (MH); Krishnagiri District: Krishnagiri, 24.09.1917, s.coll. 14907, 14926 (MH); Burgur, 19.08.1918, s.coll. 14926 (MH).

21. Eragrostis minor Host, Icon. Descr. Gram. Austriac. 4: 15. 1809.

Poa eragrostis L., Sp. Pl.: 68. 1753. Eragrostis poaeoides P. Beauv., Ess. Agrostogr.: 162. 1812, nom. nud. Lectotype: ITALY, Baeck 7, Herb. Linn. No. 87.23 (LINN), designated by Clayton in Polhill (ed.), Fl. Trop. E. Africa, Gram. 2: 234. 1974.

Eragrostis pappiana (Chiov.) Chiov. in Annuario Reale Ist. Bot. Roma 8(3): 371. 1908. Type: ERITREA, 17.03.1893, Pappy 2913 (K [K000366431, image!]).

Annual. Culms 15-56 cm high, geniculate; nodes brownish; internodes $2-4.5 \mathrm{~cm}$ long, with non-viscous glandular patches close below nodes. Leaf sheaths with crateriform glands along nerves, mouth bearded. Ligule a fringe of cilia. Leaf blades $3.5-18 \times 0.2-0.4 \mathrm{~cm}$, linear to lanceolate, margins with raised glands, upper surface scabrid. Panicles 5-15 $\times 2-3 \mathrm{~cm}$, ovate to oblong; branches alternate, stiff with crateriform glands or eglandular; pedicels stiff or slender, glandular or eglandular. Spikelets $3-20 \times 1.8-2.5 \mathrm{~mm}$, up to $45-$ flowered, ovate to lanceolate or oblong, pale green to grey with or without a purplish tinge; rachilla zigzag; florets more or less closely arranged on rachilla, disarticulating from below upwards. Glumes 1-1.75 $\mathrm{mm}$ long, subequal, ovate to lanceolate, chartaceous, 1-nerved, glandular along nerves, 1-keeled, scabrid along keel, apex acute. Lemmas $1.5-2 \times c$. $0.6 \mathrm{~mm}$, ovate to lanceolate, chartaceous, 3-nerved, glandular along midnerve, 1-keeled, scabrid along keel or or glabrous, apex acute to obtuse or subrotund. Paleas $c .1$ $\times 0.4 \mathrm{~mm}$, persistent, elliptic to oblanceolate, 2-nerved, 2-keeled, scaberulous along keels above middle, apex acute to obtuse; palea flaps narrower than its body. Anthers 3, c. $0.3 \mathrm{~mm}$ long. Caryopses $0.6-0.8 \mathrm{~mm}$ long, globose to ellipsoid or oblong, reddish brown.

Notes: Bor (1960), Jain (1968), Roy (1984) and Moulik (1997) recognized E. pappiana Chiov. as a distinct species. Though, Cope (1982) says "there is no satisfactory way to distinguish E. pappiana from $E$. minor and both species are intergrading in all the respects" he treated E. pappiana under E. minor. During the present study a specimen of E. pappiana from Jaisalmer district of Rajasthan (s.coll. 66941, housed in BSI), revealed that E. pappiana has a number of distinct features in the complete absence of glands on it and the presence of thick leaves with tubercle-based hairs. Furthermore, the spikelets of E. pappiana is linear and narrower to that of E. minor and caryopses are ovoid to ellipsoid and spindle-shaped as against the globose to oblongoid caryopses of E. minor. These features are also studied with the type and protologue of E. pappiana. However, we could not study sufficient materials of $E$. pappiana to understand its range of variation thus hesitant to admit its species status in this work.

Key to the varieties

1a. Spikelets up to $9 \times 2.5 \mathrm{~mm}$, oblong; lemma apex obtuse

1b. Spikelets up to $20 \times 1.8 \mathrm{~mm}$, lanceolate; lemma apex

acute ...21.2. var. rajasthanensis 21.1. Eragrostis minor Host var. minor

(Fig. 6 \& Plate 2)

Spikelets up to $9 \times 2.5 \mathrm{~mm}$. Lemma apex obtuse.

Flowering and fruiting: June-March.

Distribution: INDIA: Almost throughout; AFRICA, AMERICA, ASIA, AUSTRALIA, EUROPE.

Habitat: Grows generally in rock crevices, open grasslands, gardens, cultivated fields, irrigated lands, and in hill tracks, up to $3000 \mathrm{~m}$ elevation.

Chromosome nos.: 2n = 40 (Tateoka, 1954; Mehra \& al., 1968), 2n = 30 (Mulay \& Jagadisan, 1956), $2 \mathrm{n}=40$ (Sokolovskaya \& Strelkova, 1969), 2n = 60 (Reeder, 1977).

Vernacular names: Dodda purlai hullu (Kannada).

Specimens examined: INDIA. Bihar: Champaran District: Motihari, 18.08. 1964, S.P. Banerjee 243 (CAL); Gaya District: Oct. 1894, Mokim 1363 (CAL). Haryana: Kurukshetra District: Without precise locality, 
26.08.1959, J.N. Vohra 9892 (BSD). Himachal Pradesh: Kinnaur District: Baspa Valley - near Forest, 25.08.1973, K.P. Janardhanan 52806 (BSD); North Western Himalaya, May 1891, J.H. Lace 600 (CAL); Panni-Burshir, Sept.1964, K.B. Brande 3128 (CAL). Jammu \& Kashmir: Kashmir, 15.07.1964, V.N. Kaul 17 (CAL); Basali-Kashmir, 26.09.1876, C.B. Clarke 31578 (CAL); Goohaw, \pm 2133 m, 14.09.1876, C.B. Clarke 31264 (CAL); Mirn-Ladakh, 08.09.1970, U.C. Bhattacharyya 41090 (CAL); Rishtwar, $\pm 1828 \mathrm{~m}$, 17.09.1876, C.B. Clarke 31345 (CAL). Jharkhand: Sahibganj District: Rajmahal, Oct. 1894, Mokim 1458 (CAL), Karnataka: Bangalore District: Bangalore, Oct. 1891, s.coll. 626 (MH); Mysore District: 06.12.1940, K.C. Jacob 20123 (MH). Madhya Pradesh: Gwalior District: 1890, C. Maries 332 (CAL); Oct. 1890, C. Maries 158 \& 21 (CAL); Mandla District: Mandla, 500 m, 02.12.1961, J. Joseph 13520 (BSA). Maharashtra: Poona District: Parvati near Poona, 04.08. 58, s.coll. 2 (CAL); Retawdi, 28.10.1961, K.P. Janardhanan 72657 (CAL); Sindked Raja Foot Hiils, 05.09.1964, K.P. Janardhanan 100270 (BSI). Pujab: Amritsar District: May 1885, C.B. Clarke 218 (CAL); Sirsa District: Chakkajhed, 30 Apr. 1962, V.J. Nair 21597 (BSD). Rajasthan: Barmer District: Luni River bed, 17.10.1975, B. V. Shetty 2206 (CAL); Randa Village-Along Chohtan Road, 20.10.1975, B.V. Shetty 2285 (CAL); Sinadavi, 06.10.1960, s.coll. 66941 (BSI); 175 m, 12.11.1981, R.P. Pandey 7920 (CAL); Bikaner District: 10.09.1975, G.P. Roy 2021 (CAL); Churu District: 19.03.1976, G.P. Roy 2483 (CAL); Ganganagar District: 300 m, 12.11.1976, B.V. Shetty 3847 (CAL); 06.10.1977, G.P. Roy 4910 (CAL); Jaipur District: Golimar Nursery, 04.02.1965, S. Sharma 1075 (CAL); Jaisalmer District: 11.10.1960, s.coll. 66941 (BSI); Jaisalmer to Ramgarh, 12.10.1960, s.coll. 67092 (BSI); Pokhran, 350 m, 24.08. 1976, B.V. Shetty 2206 (CAL); Salam Sagar, 10.11.1973, G.L. Tiwari 719 (CAL); Jalor District: 180 m, 20.09.1979, B.L. Vyas 7320 (CAL); Jodhpur District: Beriganga, 21.09.1972, B. V. Shetty 123 (CAL); Huni Riverbed side, 07.11.1972, B.V. Shetty 272 (CAL); Piavu Village, 31.10.1972, B.V. Shetty 245 (CAL); Pali District: Buri Village, 22 Apr. 1977, R.P. Pandey 4663 (CAL); Udaipur District: 08 Apr. 63, D.M. Verma 15 (CAL). Tamil Nadu: Coimbatore District: Maruthamalai, 28.06.1930, V. Narayanaswami 3260 (MH); Pollachi, 05.10.1901, C.A. Barber 3558 (MH); Perunazhi, \pm 75 m, 14.01.1989, V. Balasubramanyam 2060 (MH); Ramanathapuram District: Chithrangudi, $\pm 35 \mathrm{~m}$, 11.11.1989, V. Balasubramanyam 2138 (MH); Perunazhi, 14.01.1989, s.coll. 2060 (MH);
Vathuvarapatti, 10.03.1953, G.H. Maduram 21822 (MH); Salem District: Buddireddipatti, 20.12.1913, s.coll. $9716(\mathrm{MH})$. Uttarakhand: Dehradun District: Aug. 1892, J.S. Gamble 23859 (CAL). Uttarpradesh: Banda District: Feb. 21, A.S. Bell 3 (CAL); Janakpur, 30.05.1986, K.K. Khanna \& R. Saran 37296 (CAL); Gangetic Plain, 26.11.1888, J.F. Duthie 6599 (CAL). Mirzapur District: 08.02.1961, U.C. Bhattacharyya 12343 (BSD); Pilibhit District: 13.05.1970, C.L. Malhotra 36896 (CAL). West Bengal: Kolkata District: Kolkata, 31.08. 1884, C.B. Clarke 35804 (CAL); Subpore, July 1964, S. Kurz 58378 (MH); Malda District: Bengal Plain, 30 Apr. 1875, C.B. Clarke 23618 (CAL); Murshidabad District: s.d., Guha Bhakshi 1031 (CAL).

21.2. Eragrostis minor var. rajasthanensis Vivek, G.V.S. Murthy \& V.J. Nair in J. Econ. Taxon. Bot. 37(4): 717. 2013. Type: INDIA, Rajasthan, Barmer District, 17 October 1975, B.V. Shetty 2206 (holo \& iso CAL!).

Spikelets up to $20 \times 1.8 \mathrm{~mm}$, lanceolate. Lemma apex acute.

Flowering and fruiting: August-November.

Distribution: INDIA: Rajasthan. Endemic.

Habitat: Sandy soil of riverbeds, at $\pm 175 \mathrm{~m}$.

Etymology: The specific epithet refers the type locality, Rajasthan in India.

Notes: This taxon appears very different from E. minor var. minor in the presence of much longer linear spikelets with up to 45 florets, unequal glumes and ellipsoid light brown caryopses (Vivek \& al., 2013f).

Specimens examined: INDIA. Rajasthan: Barmer District: Without precise locality, 175 m, 12.11.1981, R.P. Pandey 7920 (CAL); Ganganagar District: Luni Riverbed, 06.10.1977, G.P. Roy 4910 (CAL); Jaisalmer District: Pokhran, 350 m, 24.08.1976, B. V. Shetty 3309 (CAL); Jalor District: Without precise locality, $180 \mathrm{~m}$, 20.09.1979, B.L. Vyas 7320 (CAL).

22. Eragrostis multicaulis Steud., Syn. Pl. Glumac. 1: 426. 1854. Lectotype: JAPAN, Buerger s.n. (L, sh. 908.972116, specimen super., designated by Veldcamp in Blumea 47(1): 181. 2002 , isolecto L [L0038584, image!]).

(Fig. 6 \& Plate 2)

Annual. Culms 9-30 cm high, slender, erect to geniculately ascending; nodes brownish. Leaf sheaths c. $1.3 \mathrm{~cm}$ long, eglandular, glabrous or shortly hairy, mouth glabrous. Ligule up to $0.25 \mathrm{~mm}$ long, a fringe of 
cilia. Leaf blades $1.5-9 \times c .0 .15 \mathrm{~cm}$ long, linear to lanceolate, base more or less rounded, margins eglandular. Panicles 3-9 $\times 0.8-3 \mathrm{~cm}$, open or narrowly contracted; branches $1.4-5.2 \mathrm{~cm}$ long, alternate; axils glabrous. Spikelets $2-4.75 \times 1.25-1.75 \mathrm{~mm}, 4-8-$ flowered, linear to lanceolate, greyish green; pedicels 1-3 mm long, eglandular; rachilla persistent, slender, zigzag; florets loosely arranged on rachilla, disarticulating from below upwards. Glumes ovate to lanceolate, chartaceous, nerveless or 1-nerved, slightly keeled and scabrid along keel, apex acute; lower glume 0.3-0.7 mm long; upper glume $0.8-1.25 \mathrm{~mm}$ long. Lemmas $1.5-1.85 \times c .0 .6 \mathrm{~mm}$, ovate to lanceolate, 3 nerved, 1-keeled, scabrous towards apex or glabrous, apex acute or subacuminate. Paleas $1.3-1.5 \times c .0 .3 \mathrm{~mm}$, persistent, slightly curved, 2 -nerved, 2 -keeled, scaberulous along keels above middle, apex acute to obtuse; palea flaps almost equal in width to its body. Anthers 3, 0.2-0.3 mm long, pinkish to yellowish. Caryopses $0.5-0.8 \times$ c. $0.3 \mathrm{~mm}$, ellipsoid to oblongoid, obliquely truncate at base, reddish brown.

Flowering and fruiting: August.

Distribution: INDIA: Karnataka, Maharashtra, Rajasthan, Sikkim, Tamil Nadu, Uttarakhand and Uttar Pradesh; SOUTHEAST ASIA to JAPAN; introduced in EUROPE and NORTH AMERICA.

Habitat: On hillslopes, up to $2000 \mathrm{~m}$.

Chromosome no.: 2n = 40 (Ono \& Tateoka, 1953; Tateoka, 1967).

Notes: It is morphologically similar to E. pilosa but different by having solitary lower branches of panicles which are not in whorls. In E. pilosa the lower branches of panicle are in whorls and start dividing from near base.

Specimens examined: INDIA. Uttarakhand: Gharwal, s.d., G. King s.n. (CAL); Mussorie nearby, 1869, G. King s.n. (CAL).

23. Eragrostis nigra Nees ex Steud., Syn. Pl. Glumac. 1: 267. 1854. Lectotype: INDIA, Peninsular India Orientalis, Herb. Wight proper 1782 (lecto LE, isolecto $\mathrm{K})$, designated by Veldkamp in Blumea 47(1): 181. 2002.

Eragrostis atropurpurea Hochst. ex Steud., Syn. Pl. Glumac. 1: 267. 1854. Type: INDIA, Tamil Nadu, Coimbatore, Nilgiri, R.F. Hohenacker 938 (iso BM, E, Gn.v.).

(Fig. 6 \& Plate 4)
Perennial. Culms 3-90 cm high, loosely tufted, erect to geniculate; nodes brownish. Leaf sheaths $c .5 \mathrm{~cm}$ long, ciliate along one margin or glabrous, mouth bearded. Ligule a fringe of cilia. Leaf blades 8-44 × 0.25-0.55 $\mathrm{mm}$, linear to lanceolate, sparsely long hairy on surfaces. Panicles $10-24 \times 3-10 \mathrm{~cm}$, oblong to ovate, lax to effuse; branches alternate to subwhorled, capillary, flexuous; axils long ciliate, at times glabrous; pedicels 2-10 mm long, capillary, flexuous, scabrous along angles. Spikelets 3-6 × 1-2 mm, up to 8-flowered, ovate to lanceolate, black or greenish black; rachilla slender, zigzag; florets more or less closely arranged on rachilla, disarticulating from below upwards. Glumes divergent at maturity, subequal, ovate to lanceolate, chartaceous, 1-nerved, 1-keeled, scabrous along keel, apex acuminate, lower glume 1.5-2.5 mm long; upper glume $1.8-2.5 \mathrm{~mm}$ long. Lemmas $2-2.5 \times 0.8-1.2 \mathrm{~mm}$, ovate to oblong, chartaceous, 3-nerved, 1-keeled, scabrous along keel or glabrous, apex acuminate. Paleas 1.8-2.3 $\times 0.5-0.7 \mathrm{~mm}$, subpersistent, elliptic to oblanceolate, 2-nerved, 2-keeled, scaberulous along keels above middle, apex acute, margins entire; palea flaps almost equal in width to its body. Anthers 3, c. $0.2 \mathrm{~mm}$ long, purplish. Caryopses $0.4-0.6 \mathrm{~mm}$ long, oblong, ventrally flattened to grooved, dark reddish.

Flowering and fruiting: January-November.

Distribution: India: Almost throughout; CHINA, INDONESIA and SRI LANKA.

Habitat: On roadsides, stream banks, as weed in tea plantations, usually in higher elevation up to $2800 \mathrm{~m}$.

Chromosome nos.: 2n = 40 (Mehra \& al., 1968), 2n = 60 (Christopher, 1976).

Notes: A showy species with two forms in India which occurs in higher elevations. The common form is taller with spreading panicle, long-capillary and flexuous branches and pedicels having comparatively smaller spikelets. Second form is comparatively shorter with somewhat stiff panicle branches and larger spikelets. Its distant similarity with E. minor creates at times confusion in the identity. But E. minor is a glandular species with acute glumes and subglobose caryopses unlike E. nigra, which is eglandular with acuminate glumes, truncate and ventrally flattened to grooved cayopses.

Specimens examined: INDIA. Arunachal Pradesh: Dibang Valley, 09 Apr. 1999, M. Bhoumik 2351 (CAL); 15.11.1999, M. Bhoumik 2582 (CAL); 01 Apr. 2001, M. Bhoumik 3641 (CAL); Hunli-Dinbang Valley, 

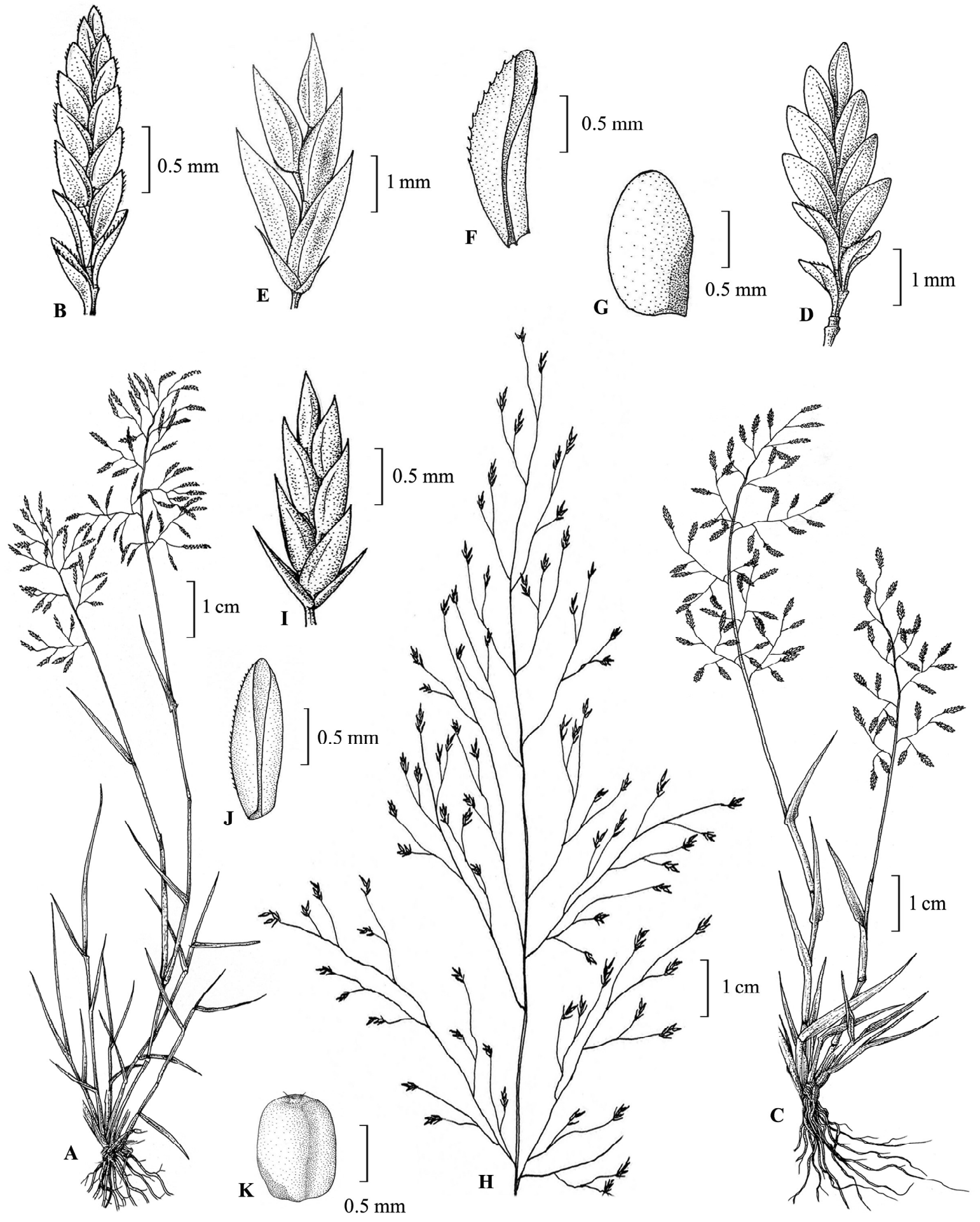

Fig. 6. Eragrostis maderaspatana Bor (A-B). A. Habit; B. Spikelet. E. minor Host var. minor (C-D). C. Habit; D. Spikelet. E. multicaulis Steud. (E-G). E. Spikelet; F. Palea-lateral view; G. Caryopsis. E. nigra Nees ex Steud. (H-K). H. Inflorescence; I. Spikelet; J. Palea-ventral view; K. Caryopsis. 
21.08.2000, M. Bhoumik 2977 (CAL); 03.09.2000, M. Bhoumik 3488 (CAL). Himachal Pradesh: Chamba District: Dalhousie, 14.09.1874, C.B. Clarke 22424 (CAL); 27.09.1874, C.B. Clarke 28183 (CAL); Shimla, 29.06.1877, J.S. Gamble s.n. (MH); Sirmaur District: Haripurdhar, 02.08.1986, R.S. Karki 32166 (BSD); Solan District: Kandaghat, November 1994, Bipin Balodi 88839 (BSD). Jammu and Kashmir: Udhampur District: Udhampur city, 07.10.1986, Ajay Swami 642 (BSD). Kerala: Idukki District: Munnar, 30.05.1944, K.C. Jacob 20488 (MH); Kottayam District: Devicolom-Upper Vagavuarai, 1800 m, 08.08.1967, B.V. Shetty 28337 (CAL, MH). Madhya Pradesh: Raigarh District: Champa, 12.02.1974, N.C. Rathakrishnan 19843 (BSA). Manipur, Barak, Nov. 1907, A. Meebold 6059 (CAL). Meghalaya: East Khasi Hills District: Shillong, 01.08.1885, C.B. Clarke 38406 (CAL); ShillongLythlingko, 27.01.1955, M.P. Guha 48 (CAL); Shillong Hills, 28.10.1938, K. Biswas 3757 (CAL); Nirala Compound - Shillong, 29.05.1956, R.S. Rao 2670 (CAL); Jaintia Hills District: Without precise locality, 15.06.1958, G. Panigrahi 16206 (CAL); Khasia Hills District: Dumpep-30.05.1911, I.H. Burkill \& S.C. Banerjee 34280 (CAL); Khasia Hills, 08.10.1914, Upendranath Kanjilal 4490 (CAL); Khasiya Hills, 30.05.1911, I.H. Burkill \& S.C. Banerjee 34280 (CAL). Odisha: Ganjam District: Kuttadya Hills, 19.08.1931, V. Narayanswami 5917 (MH). Sikkim: East Sikkim, Deorali-Gangtok, 20.11.1980, P. Chakrabarthy 904 (BSHC); Meriang sorroundings - on way to Bhusuk, 09.05.1984, S. Singh 2963 (BSHC); Padamchen, 26.06.1988, B. Krishna 8741 (BSHC); Tashi view pointon way, 28.06.1983, B. Krishna 2705 (BSHC); North Sikkim, 26.07.1990, S. Kumar \& P. Singh 9577 (BSHC); On way to Sada, 11.05.1991, R.C. Srivastava 13196 (BSHC); South Sikkim, Kabre busty, 20.05.1992, R.C. Srivastava 14507 (BSHC); West Sikkim, Hilly to Ribdi, 13.05.1995, P. Singh \& S.K. Rai 17098 (BSHC). Tamil Nadu: Coimbatore District: Kallar, 10.07.2010, C.P. Vivek 126129 (MH); Kallar R.F., 1066 m, 01.03.1942, S.R. Raju 20306 (MH); Kottadi, 1066 m, 11.03.1931, K.C. Jacob 275 (MH); Dindigul District: Kodai to Hukkal, 17.07.1920, s.coll. 16516 (MH); PumbaraiPulneys, 21.07.1920, s.coll. 16637 (MH); KodaikanalMount Pleasant, 10.07.1898, s.coll. s.n. (MH); 10.09.1905, C.A. Barber 7233 (MH); Madurai District: Without precise locality, March 1907, J. Marek 11 (CAL); Nilgiris District: Without precise locality, 13.03.1870, C.B. Clarke 10759 (CAL); 15.05.1957, K.M. Sebastine 3257 (CAL); Carrington to Kinnakurai, 2025 $\mathrm{m}, 18.06 .1970$, B.V. Shetty 34300 (MH); Coonoor, \pm 1828 m, June 1883, J.S. Gamble 12132 (CAL \& DD); Dodabetta, 17.07.2010, C.P. Vivek 126136 (MH); Dodabetta R.F., 2575 m, 28.02.1972, B.D. Sharma 40333 (MH); Hulical Droog, 1733m, 27.08. 1957, K.M. Sebastine 4124 (CAL \& MH); Kailas Pillai Estate to Sirur-Near Ebanad, 1550 m, 06.09.1970, G. V. Subbarao 36532 (MH); Kotagiri, July 1916, L.J. Sedgwick 1590 (CAL); Kunna Combai, 2000 m, 01.09.1957, K.M. Sebastine $3986(\mathrm{MH}) ; 15.06 .1883$, s.coll. s.n. (MH); June 1884, J.S. Gamble 14398 (DD); Ootacamund, 03.11.1888, s.coll. 54 (MH); 26.08. 1901, C.A. Barber $3461(\mathrm{MH}) ; 09.01 .1902$, s.coll. $4158(\mathrm{MH}) ; 11.09 .1918$, s.coll. 15541 (MH); 13.09.1918, s.coll. s.n. (MH); Sep. 1956, s.coll. s.n. (MH); Ooty, 2400 m, 24.01.1957, K.M. Sebastine 2234 (CAL \& MH); Ooty Botanical Garden, 17.07.2010, C.P. Vivek 126135; 100 m, 22.10.2011, C.P. Vivek 126155 (MH); Ootty-Governor Shola, $2225 \mathrm{~m}$, 09.07.1970, J.L. Ellis 34557 (MH); Picnic park, 2100 m, 15.05.1957, K.M. Sebastine 3257 (MH); Pykara, 13.09.1918, s.coll. 15566 (MH); 609 m, 12.09.1930, V. Narayanaswami 4230 (MH); 24.01.1957, K.M. Sebastine 2234 (MH); Sullikodu R.F.-Kotagiri, 2200 m, 28.07.1970, E. Vajravelu $35140(\mathrm{MH})$; Upper Bhavani, $2300 \mathrm{~m}, 08.06 .1970$, B. V. Shetty 34147 (MH); Salem District: Yercaud-Shevaroy Bauxite Hills, 1470 m, 19.06.1965, A.V.N. Rao 26772 (MH). Uttarakhand: Champawat District: Kumaon, 03.08. 1922, C.M. Arora 49592 (BSD); Chamoli District: s.dat., B.D. Naithani 53841 (BSD); 12.08. 1988, P.K. Hajra 37045 (BSD); Malari on way, s.dat., P.K. Hajra 87163 (BSD); Dehra Dun District: Caikur Ladwakot-Haldwadi, 20.08.1977, S.K. Murti \& R. Prasad 61951 (BSD); Sahariadhava, 18.09.1964, S.K. Malhotra 34845 (BSD); Nainital District: Laria Kantha, 2100-2800 m, 11.10.1957, T.A. Rao 4987 (BSD); Pithoragarh District: Danadhar, 14.09.1983, Bipin Balodi 75215 (BSD); Khalaytop, 13.09.1983, Bipin Balodi 75134 (BSD); Shander top hill, 2100 m, 03.06.1979, C.M. Arora 66298 (BSD); Thehri Garhwal District: s.dat., B.D. Naithani 3841 (BSD); P.K. Hajra 87163 (BSD); 11.06.1959, 2500 m, M.A. Rao 10142 (BSD); Bhyundar Valley, $2700 \mathrm{~m}$, 19.10.1962, U.C. Bhattacharya 24543 (BSD); Deolsari, 29.07.1964, U.C. Bhattacharya 33799 (BSD); Ganges Valley, 15.06.1883, J.F. Duthie 4 (CAL); Govana, 1400 m, 29.05.1979, A.K. Goel 67723 (BSD); Urni, 27.09.2000, B.P. Uniyal 96943 (BSD); Uttarkashi District: Barkot, 29.09.1995, Surendra Singh 90175 (BSD). Uttarpradesh: Bodyar, June 1894, J.S. Gamble 24840 (CAL); Dhauli Valley, 07.08.1886, J.F.Duthie 6153 (CAL); Dhurmasala, 17.10.1874, C.B. Clarke 23996 (CAL). West Bengal: Darjeeling District: June 
1881, J.S. Gamble 9402 (CAL); Darjeeling, 28.05.1947, K. Biswas 2477 \& 7479 (CAL); 16 June 1960, J.K. Maheshwari 4040 (CAL); 05.09.1869, C.B. Clarke 9016 (CAL); 24.05.1884, C.B. Clarke 35724 (CAL); Dow Hills, 23.05.1966, D. Das 153 (CAL); KakjhoraDarjeeling, 28.10.1941, K. Biswas 5753 (CAL); Kurseong, Jan. 1875, C.B. Clarke 26633 (CAL); Munsong, 28 Apr. 1965, H. Santapau 212 (CAL).

24. Eragrostis nilgiriensis Vivek, G.V.S. Murthy \& V.J. Nair in Nord. J. Botany 31(6) 700. 2013. Type: INDIA, Tamil Nadu, Nilgiri District, Coonoor $\left(10^{\circ} 20237.133\right.$ N, $76^{\circ} 47240.983$ E) $1700 \mathrm{~m}, 22$ October 2011, C.P. Vivek 126153. (holo CAL!, iso MH!).

(Plate 4)

Annual. Culms 5-35 cm high, erect or geniculate; nodes brownish to purplish; internodes $1-7 \mathrm{~cm}$ long with non-viscous glandular ring or patch close below nodes. Leaf sheaths $c .5 \mathrm{~cm}$ long, one margin ciliate, mouth bearded. Ligule up to $0.5 \mathrm{~mm}$ long, a fringe of cilia. Leaf blades 3-7 $\times 0.2-0.45 \mathrm{~cm}$, linear to lanceolate, upper surface sparsely ciliate, lower surface glabrous. Panicles 3.5-10 × 1.5-4 cm, with non-viscous glandular ring on peduncle close below panicle, ovate to oblong; branches up to $4 \mathrm{~cm}$ long, alternate with punctate glands, scabrous along angles; axils glabrous; pedicels $1-3 \mathrm{~mm}$ long, stiff, with punctate glands, scabrous along angles. Spikelets 4-13 × 1-1.65 mm, 3-20 (-28)flowered, linear to lanceolate to narrowly oblong, dark green to grey or blackish; rachilla slender, narrowly zig zag; florets closely arranged on rachilla, disarticulating from below upwards. Glumes unequal, ovate to lanceolate, membranous to chartaceous, 1nerved, 1-keeled, scabrous along keel, apex acute; lower glume $0.7-1.1 \mathrm{~mm}$ long; upper glume $1-1.5 \mathrm{~mm}$ long. Lemmas $1.3-1.8 \mathrm{~mm}$ long, elliptic to narrowly ovate, chartaceous, 3-nerved, 1-keeled, scabrous along keel towards apex, apex acute. Paleas 1.3-1.7 mm long, persistent, elliptic to oblanceolate, 2-nerved, 2-keeled, scaberulous along keels above middle, apex acute to obtuse; palea flaps almost equal in width to its body. Anthers 3, 0.2-0.3 mm long, purplish to cream coloured. Caryopses $0.45-0.7 \times 0.3-0.4 \mathrm{~mm}$, ovoid to subglobose or ellipsoid or narrowly oblong, at times narrowly flattened ventrally, yellowish brown.

Flowering and fruiting: September-November.

Distribution: INDIA: Tamil Nadu. Endemic.

Habitat: Mountainous and hilly areas in black soil, at $c$. $1700 \mathrm{~m}$ elevation.

Etymology: The specific epithet refers to Nilgiri hills, the type locality of the species in Tamil Nadu, India.

Notes: E. nilgiriensis has close similarity with stout form of $E$. nigra but differs mainly in the presence of glands, narrow spikelets, lesser florets, shorter and acute glumes and lemmas and subglobose to ellipsoid caryopses. This species is also similar to E. minor, but plants of E.nilgiriensis are less glandular, the leaf margins are eglandular and lemmas are acute at apex (Vivek \& al., 2013a).

Specimens examined: INDIA. Tamil Nadu: Nilgiri District: Lower Tiger Shola, 1666 m, 04.12.1957, K.M. Sebastine 4764 (CAL \& MH); Nilgiri, May 1889, J.S. Gamble 20841 (MH).

25. Eragrostis nutans (Retz.) Nees ex Wight \& Arn. in Wight's Cat.: 105. 1834.

Poa nutans Retz., Observ. Bot. 4: 19. 1786-1787. Type: INDIA, Tranquebaria, Koenig s.n. (BM).

Eragrostis elegantula (Kunth) Nees ex Steud., Syn. Pl. Glumac.1: 266. 1854. Type: INDIA, Wight s.n. (Herb. Wight. propr. No. 1781, K000245091 image!).

Eragrostis stenophylla sensu Stapf in Hook. f., Fl. Brit. India 7: 318. 1896, p.p., non Hochst. ex Miq., 1851.

(Fig. 7 \& Plate 2)

Perennial. Culms $25-70 \mathrm{~cm}$ high, geniculate; nodes brownish; internodes 3-8 cm long. Leaf sheaths 2-4 $\mathrm{cm}$ long, mouth bearded. Ligule up to $0.3 \mathrm{~mm}$ long, a ciliate rim with narrow membrane at base, ciliate behind. Leaf blades $4-7 \times 0.2-0.4 \mathrm{~cm}$, linear to lanceolate, upper surface scabrid. Panicles 4-14 $\times 1-$ $1.5 \mathrm{~cm}$, more or less contracted; branches $1-4.5 \mathrm{~cm}$ long, alternate, scabrid along angles; axils glabrous; pedicels $0.5-1.5 \mathrm{~mm}$ long. Spikelets $4.5-9 \times 1-1.2 \mathrm{~mm}$, 8-12-flowered, elliptic to lanceolate, dull green to greyish; rachilla zigzag; florets closely arranged on rachilla, disarticulating from below upwards. Glumes linear to lanceolate, 1-nerved, 1-keeled, scabrid along keel towards apex, apex acute; lower glume $0.7-0.8 \times$ $0.3-0.4 \mathrm{~mm}$; upper glume $0.9-1 \times 0.3-0.4 \mathrm{~mm}$. Lemmas $1-1.3 \times 0.4-0.5 \mathrm{~mm}$, ovate to lanceolate or elliptic, chartaceous, 3-nerved, 1-keeled, scabrid along keel towards apex, apex acute. Paleas $0.9-1 \times 0.3-0.4 \mathrm{~mm}$, persistent, elliptic to lanceolate, 2-nerved, 2-keeled, scaberulous along keel, apex acute to obtuse; palea flaps narrower than its body. Anthers 2 or 3, $0.2-0.3 \mathrm{~mm}$ long, purplish or brownish. Caryopses c. $0.3 \times 0.2 \mathrm{~mm}$, globose to ellipsoid, deep brown.

Flowering and fruiting: Almost throughout the year. 

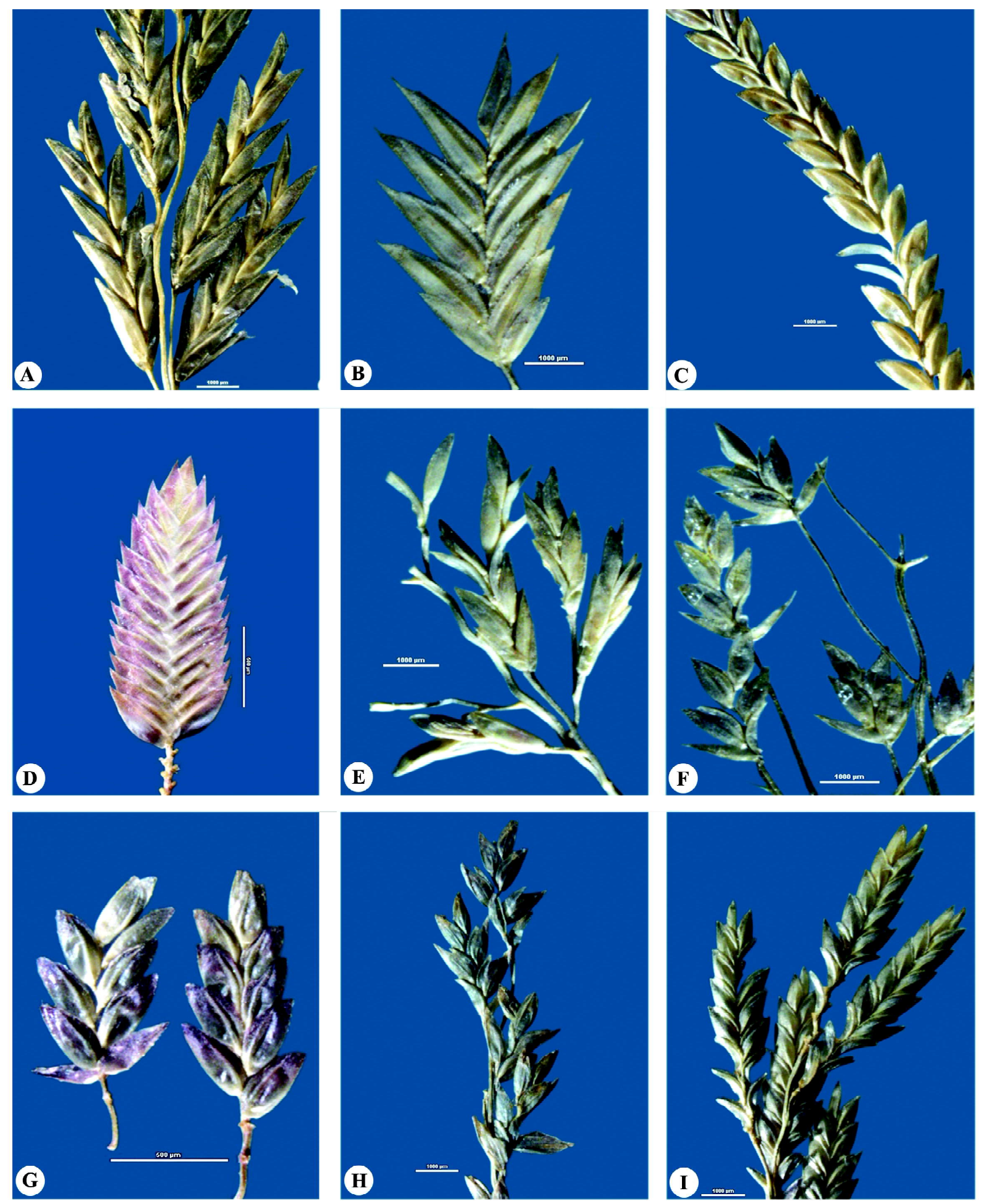

Plate 2. Spikelets of : A. Eragrostis curvula (Schrad.) Nees; B. E. deccanensis Bor; C. E. henryi Vivek, G.V.S. Murthy \& V.J. Nair; D. E. jainii Vivek, G.V.S. Murty \& V.J. Nair; E. E. lehmanniana Nees; F. E. macilenta (A. Rich.) Steud.; G. E. minor Host var. minor; H. E. multicaulis Steud.; I. E. nutans (Retz.) Nees ex Wight \& Arn. 
Distribution: INDIA: Andhra Pradesh, Bihar, Gujarat, Karnataka, Kerala, Madhya Pradesh, Maharashtra, Odisha, Rajasthan, Sikkim, Tamil Nadu, Uttar Pradesh and West Bengal; CHINA MALYSIA, SRI LANKA and throughout SOUTH EAST ASIA.

Habitat: In cultivated fields, along forest margins, river banks, occasional along the bunds of paddy fields and margins of scrub jungles, up to $c .325 \mathrm{~m}$ elevation.

Chromosome no.: 2n=60 (Chen \& Hsu, 1962; Christopher \& Abraham, 1974; Gould \& Soderstrom, 1974).

Vernacular names: Nakurmaral, Pedda garikai, Urenkai (Telugu); Looha bena (Urdu).

Notes: The combination made by Nees for this species is validated by Steudel (1840) and Wight and Arnott (1834). Hence, the correct combination is E. nutans (Retz.) Nees ex Wight \& Arn. as per the rule of priority. The author citation hitherto followed in various floras thus needs correction (see Noltie, 2005).

Specimens examined: INDIA. Andhra Pradesh: Chittoor District: Kambakam-Foot Hills, 26.09.1974, M. Chandrabose 45128 (MH); East Godavari District: Bikkavolu, 07.06.1902, C.A. Barber 4371 (MH); Devandiarum, 02.09.1907, s.coll. 8254 (MH); Samalkota, 7.08.1902, C.A. Barber 4533 (MH); 12.01.1917, s.coll. $14178(\mathrm{MH})$; Krishna District: Ivanna, 05.02.1986, P. Venkanna 5921 (MH); Mylavaram, 05.08.1907, C.A. Barber 8168 (MH); Nellore District: Kavali, 29.01.1929, K.C. Jacob 18374 (MH); Visakhapatnam District: Palasamedu, 15.10.1930, V. Narayanaswami 4631 (MH); West Godavari District: Palagudam, 08.10.1930, V. Narayanswami 4479 (MH); Rajavommagi, 27.01.1916, s.coll. $12643(\mathrm{MH})$. Kerala: Kollam District: Kollam, 22.05.1978, C.N. Mohanan 55729 (MH); Thiruvananthapuram District: Kottur R.F., 04 Apr. 1973, J. Joseph 44024 (MH); Veli, 30 m, 22.05.1978, M. Mohanan 54886 (MH). Odisha: Ganjam District: Pathwa-Gopallur Road Crossing, 20.10.1930, V. Narayanaswami 4687 (MH). Tamil Nadu: Chengalpet District: Kambakkam Hills, 05.05.1913, s.coll. 7855 (MH); Ponneri, 15.01.1917, s.coll. 14185 (MH); Cuddalore District: Cudallore-Marakkanam Road, 19.09.1979, K. Ramamurthy 64176 (MH); Pelakuppam, 19.08.1930, V. Narayanaswami 4050 (MH); Kanyakumari District: Cape Comorin, 01.11.56, S.C. Agarwal 730 \& 736 (CAL); Krishnagiri District: Hosur Cattle Farm, 914 m, 07.06.1930, V. Narayanaswamy 2998 (MH); Madurai District: Kanavai-Alagar Hills,
12.06.1957, K. Subramanyam 3449 (MH); Ramanathapuram District: Mathur-Karaikkudi, 28.11.1977, N.C. Nair 51765 (MH); Muthukulathar, 75 m, 01.01.88, V. Balasubramanyam 1496 (MH); Panagudi Forest-Sivaganga, 13.08.1964, K. Ramamurthy 20297 (CAL \& MH); Vadavayal, 08,04.1988, V. Balasubramanyam 1706 (MH); Thanjavur District: Vallum, 07.01.1989, S. Ragupathy 918 (MH); Sivasamudram, 12.05.1914, s.coll. 10406 (MH); Tirunelveli District: Courtallam, 25.10.1919, K.C. Jacob 16245 (MH); Courtallam-On the way to river falls, 333 m, 25.07.1957, K. Subramanyam 3809 (MH); Mundonthorai Ghat, 14.05.1901, s.coll. 2774 (MH). West Bengal: Bankuara District: 28.09.1965, M.N. Sanyal 815 (CAL); Hooghly District: 27.03.68, Subire Sen 477; 26.08.1968, Subire Sen 734 (CAL).

26. Eragrostis paniciformis (A. Braun) Steud., Syn. Pl. Glumac.: 268. 1854. Poa paniciformis A. Braun in Flora 24: 274. 1841. Lectotype: ETHIOPIA, 1841, A. Braun s.n. (lecto K [K000366406, image!], designated by Pradeep \& Thoiba in Rheedea 27(1): 46. 2019, isolecto K [K000366405, image!]).

Perennial. Culms up to $95 \mathrm{~cm}$ high, erect or ascending; nodes glabrous. Leaf sheaths 6-15 cm long, narrowly keeled, compressed. Ligule a rim of hairs with a narrow membranous base. Leaf blades 10-25 × 0.5-1.5 cm, flat, linear to lanceolate, upper surface hairy, lower surface glabrous. Panicles 4-22 cm long, largely open and spreading, sometimes somewhat contracted; branches alternate; axils glabrous; pedicels $0.5-15 \mathrm{~mm}$ long. Spikelets 2.5-14 × 2-5 mm, 10-26-flowered, ovate to lanceolate, greyish; rachilla persistent, zigzag; florets closely arranged on rachilla, disarticulating from below upwards. Glumes subequal, ovate to lanceolate, 1-nerved, apex acute to acuminate; lower glume 1.8-3 $\mathrm{mm}$ long; upper glume 2-3 mm long. Lemmas 2-2.7 $\mathrm{mm}$ long, oblong to lanceolate, 3-nerved, more or less gibbous, apex apiculate. Paleas $2-2.3 \mathrm{~mm}$ long, caducous, elliptic to oblong, 2-nerved, 2-keeled, ciliolate along keels. Anthers 3, 0.5-0.75 mm long. Caryopses $0.8-1.3 \mathrm{~mm}$ long, obovoid to ellipsoid, brownish.

Flowering and fruiting: October -April.

Habitat: Seasonally wet soils in jungle, swampy grasslands, streamside, wet flushes, and on roadsides, between 1075 and $2300 \mathrm{~m}$.

Distribution: INDIA. Kerala;EAST AFRICA from SUDAN, ERITREA to MALAWI and ZAMBIA. introduced in AUSTRALIA, BRAZIL, NIGER IA and VENEZUELA. 
Notes: General appearance of panicle and spikelets of E. paniciformis has some similarities with those of $E$. unioloides, but in the former the panicles are larger and spikelets are inflated. The ciliate and wingless keels of paleas are also diagnostic of E. paniciforms but they are scabrous and narrowly winged in E. unioloides.

Specimen examined: INDIA, Kerala: Idukki District: Mattupetty, $10^{\circ} 08^{\prime} 00.0^{\prime \prime} \mathrm{N}, 077^{\circ} 12^{\prime} 35.0^{\prime \prime} \mathrm{E}, 1421 \mathrm{~m}$, 16.12.2014, Thoiba \& Pradeep CU 137599 (CALI, $\mathrm{MH})$.

27. Eragrostis papposa (Roem. \& Schult.) Duf. ex Steud., Nomencl. Bot., ed. 2(1): 564. 1840. Megastachya papposa Roem. \& Schult., Syst. Veg. 2: 585. 1817, nom. illeg., pro syn. Poa papposa Duf. ex Roem. \& Schult., Syst. Veg. 2: 585. 1817. Type: SPAIN, Sagundo, Dufour s.n. (holo NTM).

(Fig.7 \& Plate 3)

Perennial. Culms 10-60 cm high, erect or ascending; nodes brownish; internodes $c .7 \mathrm{~cm}$ long. Leaf sheaths c. $4.5 \mathrm{~cm}$ long, one margin ciliate. Ligule a fringe of cilia. Leaf blades $2-15 \times 0.2-0.3 \mathrm{~cm}$, linear to lanceolate, glaucous, often form a compact cushion, margins infolded. Panicles 3-22 × 8-14 cm, open, ovate; branches up to $8 \mathrm{~cm}$ long, alternate, scabrous along angles; pedicels up to $1 \mathrm{~cm}$ long, capillary, with or without glands. Spikelets 3.5-11 × c. $1 \mathrm{~mm}$, up to 18flowered, lanceolate to elliptic, grey to green or purplish green; rachilla persistent, zigzag; florets closely arranged on rachilla, disarticulating from below upwards. Glumes ovate to lanceolate, 1-nerved, 1keeled, apex acute; lower glume $0.3-1 \mathrm{~mm}$ long; upper glume $0.8-1.3 \mathrm{~mm}$ long. Lemmas $1.1-1.7 \times c .0 .8 \mathrm{~mm}$, broadly ovate to elliptic, 3-nerved, 1-keeled, apex obtuse. Paleas $c$. $1 \times 0.3 \mathrm{~mm}$, persistent, 2-nerved, 2 keeled, scaberulous along keels above middle; palea flaps narrower than its body. Anthers 3, 0.1-0.25 mm long, yellowish. Caryopses $0.6-1.6 \times$ c. $0.3 \mathrm{~mm}$, oblong to ellipsoid, truncate at ends, ventrally flattened to grooved, yellowish brown.

Distribution: INDIA: Andhra Pradesh, Northwestern region and Sikkim; NORTH AFRICA through the MIDDLE EAST, SPAIN, SUDAN to ARABIA.

Specimens examined: INDIA. North West India: Baluchistan, s.d., Herb. R.E.P 19813 (CAL). Sikkim: North District: Ligtham, 13.09.1986, D.C.S. Raju 7321 (BSHC); West District: Gyalshing, 04 Apr. 1984, S.K. Lucksome 2824; Soreng, 14.11.81, B. Krishna 1785 (BSHC).

28. Eragrostis pilosa (L.) P. Beauv., Ess. Agrostogr.: $71,162 \& 175.1812$.
Poa pilosa L., Sp. Pl. 1: 68. 1753. Lectotype: Icon in Scheuchaen, Agrostographia; 193, t. 4. f. 3. 1719, designated by Du Puy \& al. in George \& al., Fl. Australia 50: 472. 1992. Epitype: ITALY: 9-10 Aug. 1902, A. Kneucker 344 (B [B image!]), designated by H. Scholz in Cafferty \& al. in Taxon 49: 256. 2000.

Poa indica J. Koenig ex Rottler, Neue Schriften Ges. Naturf. Freunde Berlin 4: 194. 1803. Eragrostis indica (J. Koenig ex Rottler) Willd. ex Steud., Syn. Pl. Glumac. 1: 264. 1854. Type: Rottler s.n. in Herb. Willdenow 1960/1 (holo B n.v.).

(Fig. 7 \& Plate 4)

Annual. Culms $22-70 \mathrm{~cm}$ high, slender, erect or geniculate; nodes brownish; internodes $3-9 \mathrm{~cm}$ long, glabrous. Leaf sheaths $3-8.5 \mathrm{~cm}$ long, mouth bearded. Ligule a ciliate rim with a narrow membranous base. Leaf blades $7-18 \times 0.2-0.4 \mathrm{~cm}$, linear to lanceolate, upper surface scabrid, lower surface glabrous. Panicles 9-45 × 7-10 cm, glandular or eglandular, lax, effuse; branches capillary, opposite, whorled at lower nodes; axils long-ciliate; pedicels up to $15 \mathrm{~mm}$ long, capillary, eglandular, scabrid along angles. Spikelets 3.5-6 $\times 0.7-$ $1 \mathrm{~mm}, 6-12$-flowered, linear to lanceolate, light green to grey, purple to pink-dotted towards apex; rachilla slender, zigzag; florets more or less closely arranged on rachilla, disarticulating from below upwards. Glumes linear to lanceolate, hyaline, nerveless or with an obscure nerve, apex acute to acuminate; lower glume c. $0.5 \times 0.2 \mathrm{~mm}$; upper glume $0.9-1 \times 0.3-0.4 \mathrm{~mm}$. Lemmas $1.2-1.3 \times 0.5-0.6 \mathrm{~mm}$, ovate to lanceolate, chartaceous, 3-nerved, 1-keeled, scabrid along keel towards apex, apex acute. Paleas $1-1.3 \times 0.2-0.3 \mathrm{~mm}$, persistent or sub-persistent, elliptic, 2-nerved, 2-keeled, scaberulous along keels, apex acute; palea flaps narrower than its body. Anthers 3, c. $0.2 \mathrm{~mm}$ long, purplish to yellowish or cream-coloured. Caryopses $0.4-$ $0.5 \times 0.2-0.3 \mathrm{~mm}$, ellipsoid to oblongoid, laterally flattened, base obliquely truncate, yellowish brown.

Flowering and fruiting: Almost throughout the year.

Distribution: INDIA: Almost throughout; Tropical and temperate parts of the world.

Habitat: In wet places, along ditches, margins of ponds, canals and other water-logged areas; also on sandy loam-soil, in forests, on roadsides, in open wastelands and fallow fields, up to $1800 \mathrm{~m}$.

Chromosome nos.: 2n = 40 (Ono \& Tateoka, 1953; Tateoka, 1954, 1965; Mehra \& al., 1968; Christopher \& Abraham, 1974; Jones \& al., 1978), 2n = 60 (Bowden \& Senn, 1962), 2n = 20 (Baquar \& Saeed, 1969), $2 \mathrm{n}=$ 
35 (Sokolovskaya \& Strelkova, 1969), 2n $=50$ (Mukherjee, 1977, 1978).

Vernacular name: Thodda karakai hullu (Kannada).

Notes: Eragrostis pilosa is considered as the wild progenitor of E. tef (Zucc.) Trotter. Both are morphologically similar. But $E$. tef is generally larger and mature late compared to E. pilosa. The lemmas, paleas and caryopses of $E$. tef remain attached to the rachis when mature but are sheading early in E. pilosa. There are glandular as well as eglandular forms of $E$. pilosa in India.

Specimens examined: INDIA. Andhra Pradesh: Ananthapuramu District: \pm 243 m, July 1889, J.S. Gamble 20826; \pm 457 m, 20928 (MH); Chittoor District: Chittar, 20.12.1918, s.coll. 15741 (MH); Kuppam, 25.08.1918, s.coll. 15534 (MH); Palmaner R.F., 08.10.1938, K.C. Jacob 465 (MH); Godavari District: 27.08.1902, C.A. Barber 4538 (MH); Katchalur, 01.01.1902, Bourne 3346 (CAL); Karimnagar District: Mahadevpur, 11.11.1987, M.R. Rajendraprasad 656 (MH); Kurnool District: Chelam Tank, $365 \mathrm{~m}$, 09.07.1963, J.L. Ellis 16805 (MH); Gazulapalle, 30.08.1936, K.C. Jacob (MH); Warangal District: Pakal Teak Nursery, 290 m, 14.08.1961, K.M. Sebastine 13196 (CAL \& MH); Hyderabad District: Rudoor, Nov. 1953, s.coll. s.n. (MH). Arunachal Pradesh: Dibang Valley District: 20.06.2002, M. Bhoumik 3972 (CAL); Anini, 07.09.2000, M. Bhoumik 3591 (CAL); Rheyali, 26.08.2000, M. Bhoumik 3288 (CAL). Bihar: Champaran District: Ariraj, 15.08.1964, S.P. Banerjee 165 (CAL); Gaya District: Gaya, 24.12.1876, C.B. Clarke 31819 (CAL); Manbhum District: 1888, J. Campbell 32 (CAL). Chhattisgarh: Bilaspur District: Karba, 17 Apr. 1965, G. Panigrahi \& C.M. Arora 8668 (CAL); Raipur District: Nawagaon-Raipur, 370 m, 21.08.1959, K. Subramanyam 8549 (MH). Goa: Margab, 22.08.1963, K.C. Kanodia 89457 (CAL). Gujarat: Dangs-Anwa, 03.09.1965, B. Suryanarayana 169 (CAL); 05.09.1965, B. Suryanarayana 296 (CAL); 03.09.1965, B. Suryanarayana 207(CAL); DangsDhandagad, 04.09.1965, B. Suryanarayana 236 (CAL); Dedripada-Vallabh Vidyanagar, 29.09.67, V.K. Singh 1291 (CAL). Haryana: Hissar District: Chowdhriwar, 11.05.1966, s.coll. 29779 (BSD); Pinjore District: 14.11.1959, T.A. Rao 16867 (BSD). Jammu \& Kashmir: 18.09.1876, C.B. Clarke 31409 (CAL); Nagrota, 11.08.1994, D.K. Singh \& B.P. Uniyal 88509 (BSD); Reasi, 03.091989, B.P. Uniyal 77471 (BSD); West Simapur..10.1989, W. Gillan 9120 (CAL). Jharkhand: Singhbhum District: 15.01.1903, H.H. Haines 594
(CAL). Karnataka: Bangalore District: Bangalore, January 90, s.coll. 448 (MH); Bellari District: Bellary Kote, July 1886, J.S. Gamble 17766 (CAL); Hampi, 14.10.1919, s.coll. 15982 (MH); Ramanadrug, 09.10.1919, s.coll. $15922(\mathrm{MH})$; Belgaum District: s.dat., s.coll. 849, 853 \& 854 (CAL); Bijapur District: Jamakhandi, 17.09.1979, B.R. Ramesh \& S.R. Ramesh 9373 (CAL); Coorg District: Fraserpet, 06.06.1902, C.A. Barber 4411 (MH); Mysore District: Jayachanjayapuram, 07.12.1940, K.C. Jacob 20128 (MH); Kavol R.F., 12.12.1940, s.coll. 20144 (MH). Kerala: Idukki District: High Range, 05.06.1944, K. C. Jacob 20527 (MH); Meenmutty, 800 m, 25.02.1983, C.N. Mohanan 77994 (MH); Munnar, 30.05.1944, 20482 (MH); Kollam District: Kollam, 26.11.1941, K.C. Jacob 20205 (MH); Palakkad District: Panthanthode, 825 m, 21 Aug.1966, E. Vajaravelu 27571 (CAL \& MH); Travancore, Aiyankuri, 07.09.13, M. Rama Rao 1698 (CAL). Madhya Pradesh: Chindwara District: Sillewari, 24 Aug.49, V. Narayanaswami 3606 (CAL): Dhamoh District: 09.08.78, B.K. Shukla 28651 (CAL); Gwalior District: Sheopuri National Park-Sheopuri, 14.07.1972, G. Panigrahi 16961 (CAL); Rewa District: 14.08.1986, R. Prasad 38351 (CAL); 04.08.1986, $R$. Prasad 38136 (CAL). Maharashtra: Poona District: Adbudivadi-Khed Taluka, 01.09.1962, K.P. Janardhanan 78035 (CAL); Bibi-Khed Taluka, 07.10.1962, K.P. Janardhanan 81647 (CAL); JawlidiJunnar, 01.11.1967, K. Hemadri 106863 (CAL); Junnar, 21.09.1965, K. Hemadri 104574 (CAL); PimparwadiJunnar, 05.10.1965, K. Hemadri 106833 (CAL). Pujab: s. loc., 10.09.1874, C.B. Clarke 22217 (CAL). Rajasthan: Banswar District: Shergarh, 27.08.1976, V. Singh 3069 (CAL); Bikaner District: Mud Valley, 12.09.1975, G.P. Roy 2048 (CAL); Sizbari, 23.09.1975, G.P. Roy 2128 (CAL); Churu District: Jaswantgarh College, 20.07.1976, G.P. Roy 2732 (CAL); Bamanka Bas, 27.07.1976, G.P. Roy 2769 (CAL); Ganganagar District: Bajwala, 08.10.1977, G.P. Roy 4964 (CAL); Karanpura, 27.08.1978, G.P. Roy 6439 (CAL); Nohar, 27.08.1978, G.P. Roy 6435 (CAL); Sajanda Village, 14.11.1976, G.P. Roy 3891 (CAL); Sherpura, 04.10.1977, G.P. Roy 4887 (CAL); Jaisalmer District: 08.02.1973, B.M. Wadhwa 5151 (CAL); Kakanda, 17.09.64, B.M. Wadhwa 5441 (CAL); Phalsund, 23.08.64, B.M. Wadhwa 4958 (CAL); Pokran, 24.08.64, B.M. Wadhwa 4944 (CAL); 25 Aug.1976, B.V. Shetty 3323 (CAL); Ramgarh, 02.09.64, B.M. Wadhwa 5164 (CAL); Jhujhunu District: Pilani, Nov. 1961, B.D. Deshpande 2 (BSD); Pali District: Manda Village, 21.08.1975, B.V. Shetty 1830 (CAL); Sarandana, 19.08.1975, B.V. Shetty 1806 (CAL); Tonk 


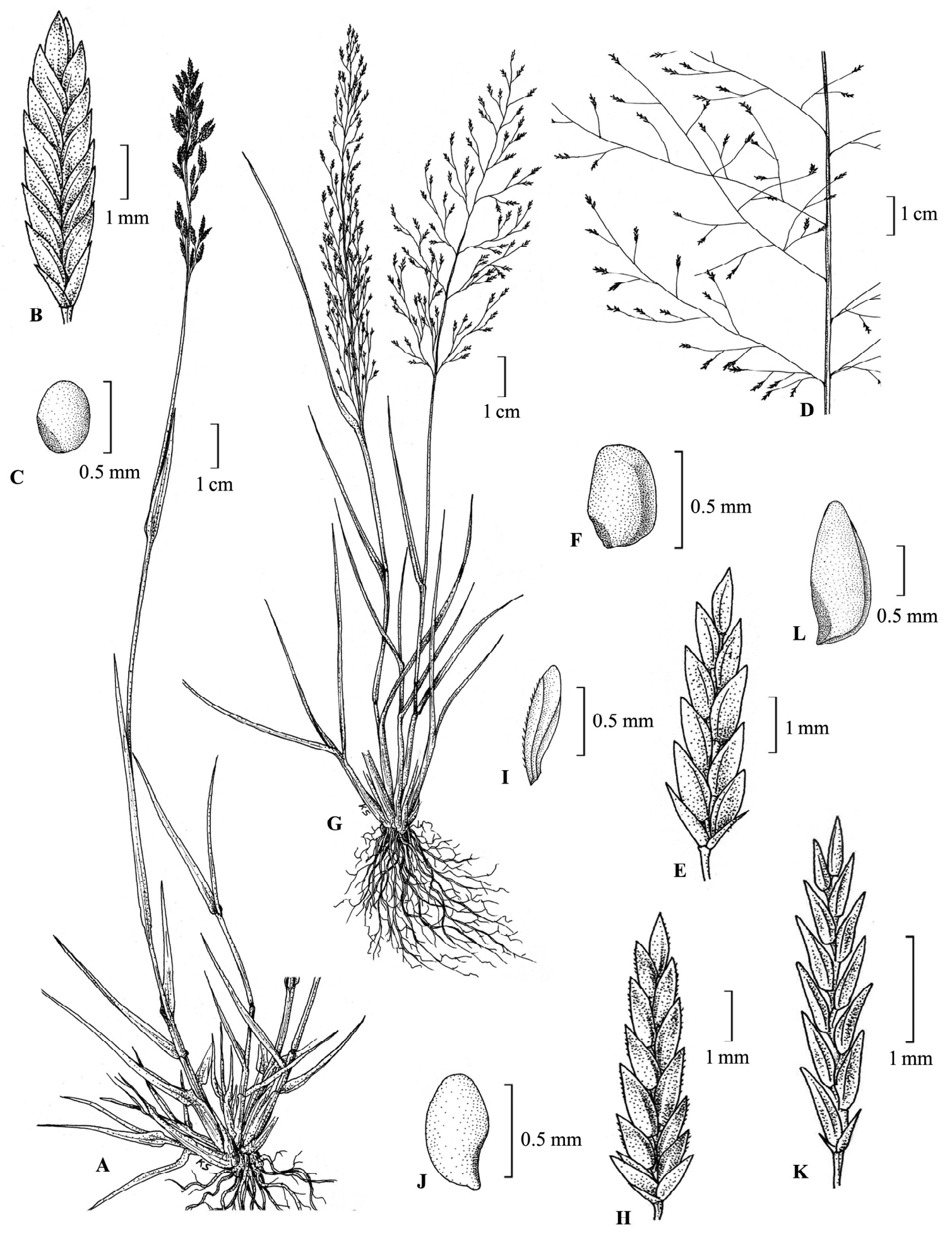

Fig. 7. Eragrostis nutans (Retz.) Nees ex Wight \& Arn. (A-C). A. Habit; B. Spikelet; C. Caryopsis. E. papposa (Roem. \& Schult.) Duf. ex Steud. (D-F). D. Inflorescence; E. Spikelet; F. Caryopsis. E. pilosa (L.) P. Beauv. (G-J). G. Habit; H. Spikelet; I. Palealateral view; J. Caryopsis. E. plana Nees (K-L). K. Spikelet; L. Caryopsis. 
District: 08.02 .1973$, B.V. Shetty 481(CAL). Tamil Nadu: Coimbatore District: Coimbatore, Aug. 1902, C.A. Barber 4890 (MH); Hassanar, $\pm 914 \mathrm{~m}, 21.06 .1929$, K.C. Jacob 18563 (MH); Kunjur, \pm 853 m, 27.01.1931, R. Raju \& Naganathan 4958 (MH); Palayamkottai, \pm 205 m, 17.11.1931, G.V. Narayana \& Naganathan 6125 (MH); Poonachi, 10.10.1901, C.A. Barber 3765 (MH); Sivasamudram, 17.02.1930, V. Narayanaswamy 20040 (MH); Thadagai Hills-Andiyur, 11.07.2010, C.P. Vivek \& S. Arumugam 126131 (MH); Walayar Dam Area, 04.09.2010, C.P. Vivek 126140 (MH); Chengalpet District: Avadi, 18.09.1917, s.coll. 14860 (MH); Cuddalore District: Gollapalle-Gudiattam, 08.10.1939, K.C. Jacob 6353 (MH); Palakuppam, 21.08.1930, V. Narayanaswami 4047 (MH); Krishnagiri District: Hosur Cattle Farm, $\pm 914 \mathrm{~m}, 09.06 .1930, V$. Narayanaswami 3041 (MH); Krishnagiri, 24.09.1917, s.coll. 14942 (MH); Madras District: Madras, Nov. 1890, s.coll. s.n. (MH); Nungampakkam, 01.11.1899, s.coll. s.n. (MH); Madurai District: Soranganar Falls-On the way, $375 \mathrm{~m}$, K. Subramanyam $8165(\mathrm{MH})$; Nilgiri District: Oct. 1883, J.S. Gamble 13051 (CAL); Bella Mogilalam, 25.12.1912, s.coll. 9830 (MH); Ootacamund, 11.09.1918, s.coll. 15540 (MH); 27.08.1901, C.A. Barber 3482 (MH); Ootty-Naduvattam Forest, 18 Aug.1889, s.coll. 15 (MH); North Arcot District: Korponur, Polur, 14.10.1939, K.C. Jacob 6371 (MH); Ranipet, 14.03.1917, s.coll. $14738(\mathrm{MH})$; Pudukkotai District: Pennamaravathi, 17.09.1985, C. Arulappan 499 (MH); Ramanathapuram District: Chithrangudi, $25 \mathrm{~m}, 11.11 .1989$, V. Balasubramanyam 2130 (MH); Kamuthakudi, 40 m, 19.09.1988, 1839 (MH); Mamarathukedai-Santhankoil, $500 \mathrm{~m}$, S.R. Srinivasan 63596 (MH); Parayankulam ForestSivaganga, $100 \mathrm{~m}, 22.08 .1964$, K. Ramamurthy 21023 (CAL \& MH); Therkumookai, sea level, 26.12.1989, V. Balasubramanyam 2326(MH); Thiruvadanai, 18.09.1988, s.coll. 1817 (MH); Salem District: Hosur, 12.12.1917, s.coll. 15433 (MH); Metigiri, 12.12.1917, s.coll. $15435(\mathrm{MH})$; Tirunelveli District: Palayamkottai, 11.05.1901, C.A. Barber 2723 (MH); Trichinapally District: Pallavalavam-Kollimalai, 23.06.1916, s.coll. s.n. (MH). Uttarakhand: Dehra Dun District: Bindal, 10.08.1965, C.R. Babu 34981 (BSD); 02.01.1966, C.R. Babu 3498 (BSD); Rampur road, 06.08.1988, U. Singh 612 (BSD). Uttarpradesh: Gangetic Plain, 28.11.1886, J.F. Duthie 6602 (CAL); Mirzapur District: 30.07.65, O.P. Misra 9999 (CAL); 12.10.1969, G. Panigrahi 1246 (CAL); Tanda Falls, $200 \mathrm{~m}, 19.09 .1961$, U.C. Bhattacharya 39403 (BSD); 06.10.1969, G. Panigrahi 12271 (CAL); Saharanpur District: 10.09.1988, J.F.
Duthie, 7710 (CAL); 26.08.1888, J.F. Duthie 7701 (CAL); Kachahari road, Aug. 1985, T.S Murthy \& A.K. Goel 1767 (BSD). West Bengal: Birbhum District: Daskalgram, 26.08.1969, B.K. Basak 1062 (CAL); Purulia District: 07.12.1894, C.B. Clarke 25244 (CAL); Mudhapoor, Alazariburgh-Chota Nagpore, 01.10.1883, C.B. Clarke 33641 (CAL).

29. Eragrostis plana Nees, Fl. Afr. Austral.: 390. 1841. Type: SOUTH AFRICA, between Gekau and Basche, J.F. Drège s.n.

(Fig. 7 \& Plate 3)

Perennial. Culms $65-100 \mathrm{~cm}$ high, erect; nodes brownish. Leaf sheaths flattened, smooth, shiny, glabrous or puberulent. Ligule $0.2-0.4 \mathrm{~mm}$ long, a fringe of cilia. Leaf blades $15-50(-70) \times 0.2-0.4 \mathrm{~cm}$, margins involute, upper surface sparsely hairy. Panicles $13-28 \times 2-8 \mathrm{~cm}$, narrowly oblong to lanceolate, contracted to open, branches $1-8 \mathrm{~cm}$ long, appressed to main axis; axils glabrous or slightly hairy. Spikelets 6-14 × 1.3-2.5 mm, 9-14-flowered, linear to oblong, greenish to greyish; rachilla narrowly zigzag with internodes $c .1 \mathrm{~mm}$ long; florets loosely arranged on rachilla, disarticulating from below upwards. Glumes narrowly ovate to lanceolate, membranous to hyaline, coriaceous, 1-nerved, glandular along nerve, 1-keeled, apex acute; lower glume $0.4-1.2 \mathrm{~mm}$ long; upper glume $1-1.5 \mathrm{~mm}$ long. Lemmas $1.8-3 \times c .0 .5 \mathrm{~mm}$, ovate to lanceolate, narrowly curved, coriaceous, 3-nerved, punctately glandular along nerves, lateral nerves distinct, 1-keeled, keel prominent, apex subacuminate to acute. Paleas $1.8-3 \times c .0 .4 \mathrm{~mm}$, persistent, lanceolate to elliptic, 2-nerved, 2-keeled, punctately glandular and scaberulous along keels above middle, apex obtuse to truncate; palea flaps almost equal in width to its body. Anthers 3, 1.2-1.8 mm, reddish to purple or yellowish. Caryopses $1-1.6 \times c$. $0.5 \mathrm{~mm}$, prismatic to ovoid or narrowly oblong, dark reddish.

Flowering and fruiting: September-May.

Distribution: INDIA: Introduced (Bor, 1960); MYANMAR, TROPICAL and SOUTH AFRICA.

Chromosome no.: $2 \mathrm{n}=20$ (Pienaar, 1955).

Notes: Bor (1960) mentioned that this species was introduced in India and neighbouring country, Myanmar. However, as we could not trace any representative specimens of this species in Indian herbaria hitherto, we include it here based on the report of Bor (l.c.). The description and illustration provided here are based on the African specimen deposited at CAL. 
Specimen studied: AFRICA. Dundee-Natal, $121 \mathrm{~m}$, Dec. 198, W.E. Green 60 (CAL).

30. Eragrostis riparia (Willd.) Nees, Fl. Bras. Enum. Pl. 2(1): 512. 1829.

Poa riparia Willd., Ges. Naturf. Freunde Berlin Neue Schriften 4: 185. 1803. Eragrostis tenella (L.) P. Beauv. var. riparia (Willd.) Stapf in Hook.f., Fl. Brit. India 7: 315. 1896. Type: INDIA, Madras, Rottler s.n. in Herb. Willdenow 1940/2 (holo B, fragm. K, image!).

(Fig. 8 \& Plate 3)

Perennial. Culms up to $40 \mathrm{~cm}$ high, geniculate; nodes brownish; internodes $c .3 \mathrm{~cm}$ long with non-viscous glandular ring close below nodes. Leaf sheaths $c .4 \mathrm{~cm}$ long, narrowly ciliate along one margin or glabrous, mouth bearded. Ligule a fringe of cilia. Leaf blades 1.6$11 \times 0.2-0.3 \mathrm{~cm}$, linear to lanceolate, upper surface scabrid. Panicles $1.3-7 \times 0.6-3 \mathrm{~cm}$, spiciform, compact with clustered spikelets, lanceolate; branches alternate to subwhorled; axils glabrous, at times minutely ciliate; pedicels $0.5-2 \mathrm{~mm}$ long. Spikelets $2.2-3 \times 1-1.2 \mathrm{~mm}$, up to 10 -flowered, ovate to lanceolate, light green to green with a purple tinge; rachilla zigzag; florets more or less closely arranged on rachilla, disarticulating from above downwards. Glumes $0.7-0.8 \times 0.3-0.4 \mathrm{~mm}$, subequal, ovate to lanceolate, chartaceous, margins entire, at times serrulate towards base, 1-nerved, 1keeled, scabrid along keel, apex acute to subacuminate. Lemmas $1-1.2 \times c .0 .4 \mathrm{~mm}$, elliptic to ovate, chartaceous, 3-nerved, 1-keeled, scabrid along keel, apex acute. Paleas $0.8-1 \times$ c. $0.4 \mathrm{~mm}$, caducous, elliptic to oblanceolate, 2-nerved, 2-keeled, ciliate along keels, apex acute to obtuse; cilia on palea keels ascending, equaling to or shorter than width of palea body; palea flaps narrower than its body, at times flaps margins negligibly hairy towards apex. Anthers 3, c. $0.2 \mathrm{~mm}$ long, purplish. Caryopses c. $0.3 \times 0.2 \mathrm{~mm}$, ellipsoid to ovoid, light brown.

Flowering and fruiting: Almost throughout the year.

Distribution: INDIA: Andhra Pradesh, Assam, Bihar, Karnataka, Kerala, Madhya Pradesh, Maharashtra, Odisha, Rajasthan, Sikkim, Tamil Nadu, Uttar Pradesh and West Bengal; MYANMAR, PAPUA NEW GUINEA, PHILIPPINES and SRI LANKA.

Habitat: Grows in red sandy soils, in drier situations on exposed hillslopes, sandy coast, near backwaters and in wastelands and roadsides, up to $c .1142 \mathrm{~m}$ elevation.

Vernacular name: Kadu kamba hullu (Kannada).
Specimens examined: INDIA. Andhra Pradesh: Ananthapuramu District: Kalasamudram R.F. Field, 08.02.1983, N. Yesoda $1166(\mathrm{MH})$; Chittoor District: Irumalai Hills, 12.10.1938, K.C. Jacob 472 (MH); East Godavari District: Mundapetta, 17.03.1902, C.A. Barber 4290 (MH); Krishna District: Allum, 17.07.1907, s.coll. s.n. (MH); 19.07.1907, s.coll. $7940(\mathrm{MH})$; Nellore District: Ramabalaka, Aug. 1883, J.S. Gamble 12683 (MH); Visakhapatnam District: Galikonda towards from First Bunglaw, 1142 m, 15.05.1964, G. V. Subbarao 19584 (CAL, MH). Karnataka: Bangalore, 04.11.90, s.coll. 534 (MH); Coondapur Taluk-Beetady, 18.02.1985, Sherif \& Suresh 364 (MH); Mangalore Taluk-Bangalore, 15.09.85, Sherif \& Suresh $644(\mathrm{MH})$; Bellari District: Itagarai, 19.09.1916, s.coll. $13783(\mathrm{MH})$; Chamarajanagar District: Hebba Halla, Anepalayam, Kollegal, 762 m, 05.07.1930, V. Narayanaswami 3584 (MH); Chitradurga District: Hosakere, 13.11.1978, K.P. Sreenath \& S.R. Ramesh 4201 (CAL); Dharmapuri District: Harur, Chitteri Hills, 11.08.1978, K.M. Matthew 16245 (RHT); Mysore District: Tunkur, 06.12.1940, K.C. Jacob 20122 (MH); Uttara Kannada District: Pilicode, 04.12.1919, s.coll. 16266 (MH). Kerala: Kollam District: Kollam-Backwaters, 22.12.1979, C.N. Mohanan 65018 (MH). Madhya Pradesh: Chatarpur District: Mohabo, 1983, B. Datt 30330 (BSA). Rajasthan: Banswara District: Ghautala, 01.01.1966, R.B. Majumdar 10223B (BSA). Tamil Nadu: Coimbatore District: Anaipodi - On the way, 24.02.1942, S.R. Raju 20284 (MH); AnepalayamHabbe Halla, 05.07.1930, V. Narayanaswami 3584 (MH); Bharathiar University Campus, 27.06.2010, C.P. Vivek 126122 (MH); Konamalai-slope, 15.11.1962, C.P. Sreemadhavan 258 (MH); Palamalai, $860 \mathrm{~m}$, 17.09.1969, M.V. Viswanathan 169 (MH); Perur Dam, Noyyil River, 633 m, 15.02.1957, K. Subramanyam 2328 (MH); Pungamgeri R.F.-Siruvani, $\pm 457 \mathrm{~m}$, 26.01.1932, M. Ramavelu 51; 68 (MH); Thekkumalai Hills, 666 m, 12.12.1956, K.M. Sebastine 1731 (MH); Chengalpet District: Vedanthangal, $\pm 120 \mathrm{~m}$, 28.01.1978, A.N. Henry 47096 (CAL \& MH); Cuddalore District: Cudallore, 05.03.1902, C.A. Barber 4263 (MH); Marakkanam-On the way, 16.02.1979, K. Ramamurthy 60230 (MH); Kanyakumari District: Kumari Amman Temple Complex, \pm 4 m, 22.07.1987, K. Ravikumar 85762 (MH); Nilgiri District: Gudallore, 05.03.1902, C.A. Barber 4263 (MH); North Arcot District: Gudiatham, 22.08.1918, s.coll. 15521 (MH); Karpudi-Polur, 14.10.1939, K.C. Jacob 6370 (MH); Ramanathapuram District: Kurusodai Islands, 30.01.1945, D. Daniel \& S.R. Raju 20082 (MH); 
Manamadurai, 125 m, 25.06.1978, N.C. Nair 57479 (MH); Paraiyankulam Forest-Sivaganga, 22.08.1964, K. Ramamurthy 21032 (CAL \& MH); Pirappanvalasai, 22.05.1988, V. Balasubramanyam 1751 (MH); Salem District: Hosur, 12.12.1917, s.coll. 15431 (MH); Hosur cattle Farm, 07.06.1930, V. Narayanaswami 2956 $(\mathrm{MH})$; Palamalai-Manguli, Pasumani, $860 \mathrm{~m}$, 14.07.1970, M.V. Viswanathan 701 (MH); Thanjavur District: Beach area in Kodikkarai R.F., 13.02.1985, K.R. Sasidharan 10989 (FRC); Muthupet, 19.09.1965, K. Ramamurthy 25865 (MH); Point Calimere, 07.08.1960, K.M. Sebastine 10612 (CAL \& MH); Velankanni, sea level, 13.11.1987, S. Ragupathy 516 (MH); Thiruchirappally District: Near Puliancholai, 08.08.1958, K.M. Sebastine 6232 (MH); Adyar,.07.1889, J.S. Gamble 20786 (MH); Tirunelveli District: Padaliarkulam Aru Banks, 04.09.1969, B.V. Shetty $32215(\mathrm{MH})$; Tirunelveli District: Kalakkadu R.F., 225 m, 12.11.1962, J. Joseph 15260 (MH); Singampatti, 300 m, 14.10.1957, K.M. Sebastine 4449 (MH); Therkkumalai Road-Courtallam, 23 Apr. 1957, K. Subramanyam 2857 (MH). West Bengal: Bankura District: 1967, s.coll. 16 (CAL).

31. Eragrostis rottleri Stapf in Hook.f., Fl. Brit. India 7: 321. 1896. Lectotype: INDIA, Tamil Nadu, Nagapattinam District, Tranquebar (Tharangampadi), 1799, Rottler s.n. (lecto K [K000518040 excluding the top left specimen, image!], designated by Vivek \& al. in Nelumbo 55: 109. 2013.

Annual. Culms $22-48 \mathrm{~cm}$ high, geniculate. Leaf sheaths glabrous, mouth glabrous. Ligule a fringe of cilia. Leaf blades $2.5-17 \mathrm{~cm}$ long, more or less convolute, upper surface scabrid. Panicles 3-15 cm long, oblong, open; branches alternate, filiform to capillary with glandular spots, lower branches start dividing from near base; pedicels of lateral spikelets very short with glandular spots. Spikelets $4-5 \times 1.2-1.5 \mathrm{~mm}, 6-12$-flowered, pale brown or white; rachilla zigzag; florets more or less closely arranged on rachilla, disarticulating from below upwards. Glumes 1-1.2 mm long, subequal, 1-nerved, 1-keeled. Lemmas 1.25-1.5 mm long, oblong, 3nerved, lateral nerves prominent, apex subacute, pale brown or white. Paleas shorter than lemmas, persistent, 2-nerved, 2-keeled, scaberulous along keels. Anthers 3, c. $0.25 \mathrm{~mm}$ long. Caryopses subglobose, $c .0 .6 \mathrm{~mm}$ long, terete.

Flowering and fruiting: July-December.

Distribution: INDIA: Tamil Nadu; PAKISTAN?

Habitat: Grows in coastal plains.
Notes: Eragrostis rottleri has some resemblance with $E$. minor and E. viscosa. It differs from E. minor mainly by the complete absence of crateriform glands and beard at the mouth of the leaf sheath (Cope, 1982; Kabeer \& Nair, 2009). Due to the presence of a viscid culm portion below the panicle, it can be easily mistaken for $E$. viscosa. But in E. rottleri the spikelets are quite different mainly in that the rachilla is persistent, the florets disarticulating from below upwards and the palea keels are scabrous. In E. viscosa, however, the rachilla and the florets disarticulating from above downwards and the palea keels are distinctly ciliate.

Eragrostis rottleri was considered earlier as endemic to Peninsular India based on its collection from Tamil Nadu. Later, Cope (1982) while working on the Poaceae for the Flora of Pakistan, came across a specimen, R.R. Stewart 26395 (K) collected from Gilgit of Pakistan, which matches very well with the type specimens (syntypes) of E. rottleri, nevertheless, he was hesitant to admit this to the flora of Pakistan based on this single collection. Therefore the endemic status of this species is doubtful.

Specimens examined: INDIA. Peninsula Ind. Orientalis: s.loc., s.d., Wight s.n., Herb. Wight. propr. no. 1785 (K000643342, E00393634, images!); Thiruvarur District: Munargudy (Mannargudi), 01.07.1824, Wight s.n., Herb. Wight propr. no. 1785 (E00393636, image!); Tirunelveli District: Palamcotta (Palayamkottai), 15.12.1835, Wight s.n., Herb. Wight propr. no. 1785 (E00393635); Tranquebar, 1799, Rottler s.n. (K000518040); Tranquebar. Oct. 1798, Rottler s.n. (K000643343, image!).

32. Eragrostis schweinfurthii Chiov. in Annuario Reale Ist. Bot. Roma 8(3): 368-369. 1908. Type: Eritrea, 23 August 1902, A. Pappi 1245 (BR [BR0000008758002, image!]).

Eragrostis chalcantha Schweinf., Samml. Arab. Aeth. Pflanz. ser. 1: 100. 1896, nom. illeg., non Trin., Mem. Acad. Imp. Sci. St.- Petersbourg, Ser. 6, Sci. Math. 1(4): 401. 1830.

Eragrostis kiwuensis Jedwabn., Bot. Arch. 5: 206. 1924. Eragrostis schweinfurthii Chiov. var. kiwuensis (Jedw.) S.M. Phillips in Kew Bull. 42(4): 930. 1987. Type: Rwanda, November 1907, J. Mildbraed 1784 (holo B [B100167419, image!], iso BR [BR0000008758149, image!]).

(Fig. 8 \& Plate 3)

Annual or perennial. Culms $10-50 \mathrm{~cm}$ high, erect to 

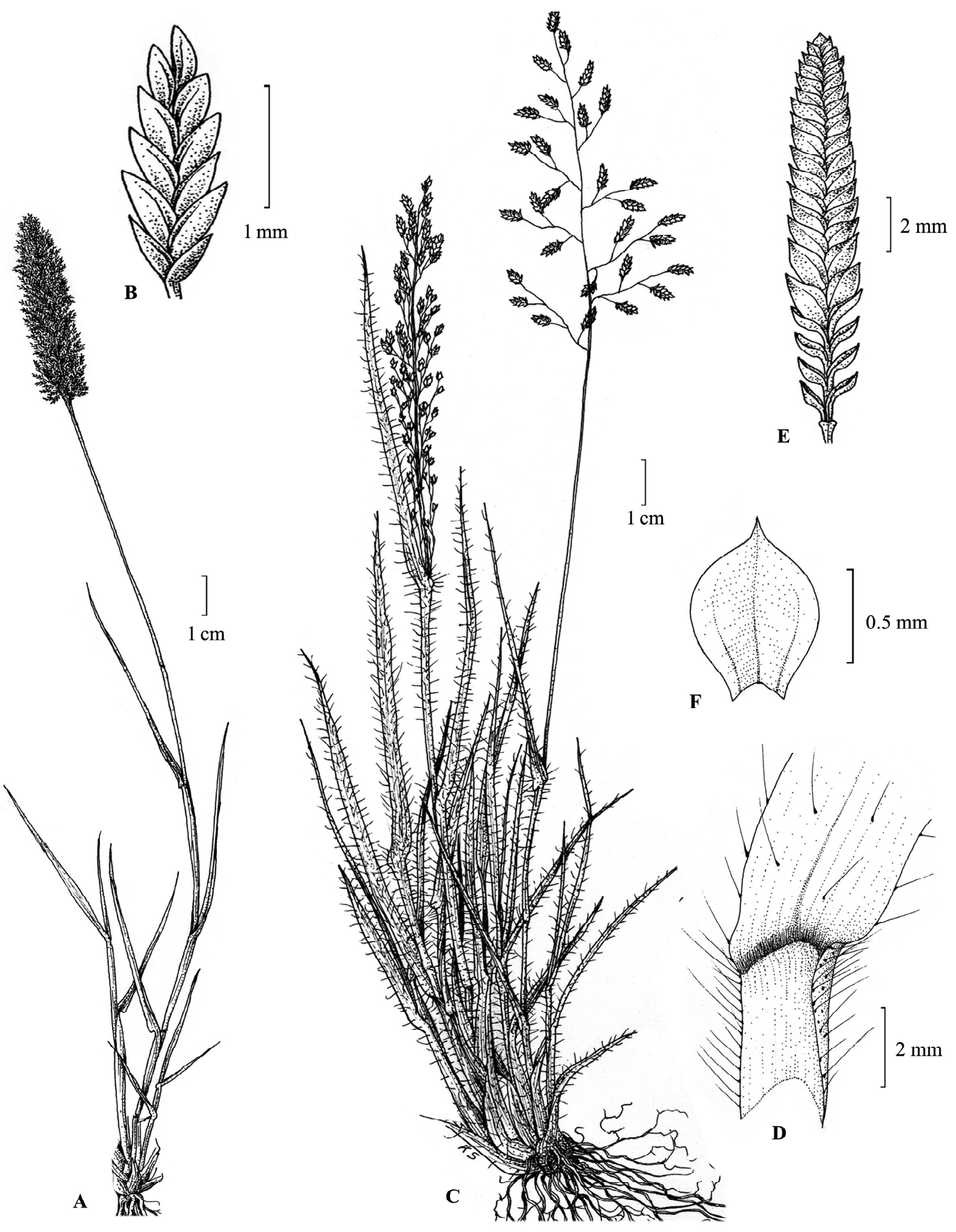

Fig. 8. Eragrostis riparia (Willd.) Nees (A-B). A. Habit; B. Spikelet. E. schweinfurthii Chiov. (C-F). C. Habit; D. Leaf sheath \& Leaf blade; E. Spikelet; F. Lemma-ventral view. 
ascending; nodes brownish; internodes $c .5 \mathrm{~cm}$ long with non-viscous glandular ring close below nodes. Leaf sheaths $c .4 \mathrm{~cm}$ long, hirsute with tubercle based cilia all over, and leaving sunken pits after falling. Ligule a ciliate rim with narrow membrane at base. Leaves mostly crowded at base; blades $4-9 \times$ c. $0.4 \mathrm{~cm}$, margins as well as both sides hirsute with tuberclebased cilia, leaving sunken pits after falling. Panicles $4-9 \times 3-4 \mathrm{~cm}$, as long as rest of plant, open, broadly ovate; primary branches alternate; axils slightly ciliate when young, glabrous at maturity; pedicels $1-2 \mathrm{~mm}$ long, terete to narrowly angled, barbellate. Spikelets 4-10 $\times 0.9-2 \mathrm{~mm}, 10-28$-flowered, ovate to oblong, dull green to grey; rachilla straight; florets firmly arranged on rachilla, disarticulating from below upwards. Glumes ovate to lanceolate, boat shaped, 1nerved, narrowly keeled, scabrous along keel, apex acute to subacuminate; lower glume $0.9-1 \times 0.4-0.6$ $\mathrm{mm}$; upper glume 1.1-1.2 $\times 0.5-0.6 \mathrm{~mm}$. Lemmas 1$1.5 \times 0.8-1 \mathrm{~mm}$, broadly ovate, boat shaped, chartaceous, 3-nerved, nerves indistinct, apex acute, acuminate from peripheral view. Paleas $1.2-1.5 \times c .0 .5$ $\mathrm{mm}$, persistent, obovate to oblanceolate, curved, chartaceous, 2-nerved, 2-keeled, scaberulous along keels, apex acute to obtuse; palea flaps narrower than its body. Anthers 2, 2-3 mm long, purplish. Caryopses c. $0.4 \mathrm{~mm}$, globose to orbicular, deep brown or reddish brown.

Flowering and fruiting: June-November.

Distribution: INDIA: Tamil Nadu; ARABIA, TROPICAL AFRICA and SRI LANKA.

Habitat: On roadsides in hill stations, up to $2300 \mathrm{~m}$.

Notes: Its range in India is limited to higher altitudes of Palni and Nilgiris in Southern part of India. It differs from all Indian Eragrostis by the presence of bulbous based hairs throughout the plant body which gives it a woolly appearance. These hairs trap the morning mist giving a beautiful appearance to the roadsides.

Specimens examined: INDIA. Tamil Nadu: Madurai District, Kodaikanal, Konalar, 05.11.1987, K.M. Mathew 51126 (RHT); Nilgiri District: Coonoor, Along railway track, $1700 \mathrm{~m}, 22.10 .2011$, C.P. Vivek 126157 (MH); Dodabetta, 27.07.2012, Remya J. \& Prasanna 75495 (TBGT).

33. Eragrostis subsecunda (Lam.) E.Fourn., Mexic. Pl. 2: 118. 1886.
Poa subsecunda Lam., Tabl. Encycl. 1: 184. 1791. Type: EAST CHINA, Herb. Lamarck, Sonnerat s.n. (holo P, photo in $\mathrm{K})$.

(Fig. 9)

Perennial. Culms $20-80 \mathrm{~cm}$ high, willowy; nodes brownish. Leaf sheaths 6-16 cm long, slightly keeled. Ligule obscure. Leaf blades 1-15 $\times 0.1-0.3 \mathrm{~cm}$, linear to lanceolate. Panicles 3-25 cm long, open, lax, loosely spiculate, subsecund. Spikelets 7-9 mm long, 10-16 or more flowered, oblong or oblong-lanceolate, purplish green; florets closely arranged on rachilla, disarticulating from below upwards. Glumes ovate to lanceolate, chartaceous, 1-nerved; lower glume 1-2 $\times$ c. $0.5 \mathrm{~mm}$; upper glume $2-2.5 \times 0.5-1 \mathrm{~mm}$. Lemmas $2-$ $2.5 \times 1-1.5 \mathrm{~mm}$, broadly ovate, chartaceous, 3-nerved, apex acute. Paleas $1.5-2 \times c .1 \mathrm{~mm}$, persistent or subpersistent, elliptic to lanceolate or oblong to lanceolate, 2-nerved, 2-keeled, ciliolate along keels above middle, apex acute to obtuse; palea flaps narrower than its body. Anthers 3, 0.25-0.5 mm long, purplish. Caryopses ovoid, 0.5-1 mm long, brownish.

Flowering and fruiting: March-September.

Distribution: INDIA: Assam, Kerala and Tamil Nadu; AUSTRALIA, BANGLADESH, EAST CHINA, HAINAN, HONG KONG, SE ASIA, SRI LANKA, TAIWAN, TIBET.

Habitat: Occasional along the banks of backwaters, streams and canals.

Notes: This species is reported from India for the first time by Sreekumar \& Nair (1991) and from Tamil Nadu by Kabeer \& Nair (2009). It is very close to E. unioloides. For this reason a number of $E$. unioloides specimens are wrongly determined as E. subsecunda in some herbaria.

Specimens examined: INDIA. Kerala: Alappuzha District: Kumarakodi, 21.09.1981, P.V. Sreekumar 70574, 70582; Pallana, 25.06.1980, P.V. Sreekumar 67646; Kollam District: Neendakara, 19.06.1980, P.V. Sreekumar 67613 (MH).

34. Eragrostis superba Peyr. in Sitzungsber., Kaiserl. Akad. Wiss., Math.-Naturwiss. Cl. 38: 584. 1860. Type: AFRICA, Angola; Benguefa ac Loanda, H. Wawra 244 (holo W, fragment (spikelets) in K). (Fig. 9 \& Plate 3)

Perennial. Culms $80-130 \mathrm{~cm}$ high, erect; nodes brownish; internodes $c .15 \mathrm{~cm}$ long, striate. Leaf sheaths $8-12 \mathrm{~cm}$ long with or without pitted glands, mouth bearded. Ligule a line of villous hairs. Leaf blades $20-$ $35 \times c .0 .4 \mathrm{~cm}$, linear to lanceolate, upper surface scabrid. 
Panicles 26-28 × 3-4 cm, linear; primary branches alternate; axils glabrous; pedicels $3-5 \mathrm{~mm}$ long. Spikelets 9-11 ×8-9 mm, 8-15-flowered, broadly ovate to oblong, yellowish green; rachilla zigzag or nearly straight; florets firmly arranged on rachilla, disarticulating entire at maturity. Glumes linear to lanceolate, coriaceous, 1-nerved, nerve thick and distinct, 1-keeled, keel narrowly winged and scabrous towards apex, apex acuminate; lower glume 3-4.3 $\times$ $0.8-1.2 \mathrm{~mm}$; upper glume $3.8-4.3 \times 0.8-1.3 \mathrm{~mm}$. Lemmas $4.2-4.5 \times 1.3-1.6 \mathrm{~mm}$, ovate to lanceolate, boat shaped, coriaceous, 3-nerved, nerves prominent, midnerve very thick and keeled, keel narrowly winged towards apex, apex acuminate. Paleas 4-4.3 × 1-1.4 $\mathrm{mm}$, persistent, ovate to lanceolate, chartaceous to coriaceous, 2-nerved, nerves thick, 2-keeled, keels broadly winged and scaberulous, apex acuminate; palea flaps narrower than body of palea. Anthers 3, 2-2.2 $\mathrm{mm}$ long, brownish. Caryopses 1-1.5 mm long, ellipsoid, deep brown.

Flowering and fruiting: March-September.

Distribution: INDIA: Karnataka and Tamil Nadu; TROPICAL AFRICA, PAKISTAN.

Habitat: Grows on rocky slopes and roadsides.

Chromosome nos.: $2 \mathrm{n}=40$ (Moffett \& Hurcombe, 1949; Mehra \& al., 1968; Shanthamma \& Narayan, 19761977; Reeder, 1977), 2n = 20 (Dujardin, 1978).

Notes: The larger spikelets and prominently winged palea keels are the unique features of this sepcies. The spikelets also disarticulate below the glumes and fall entirely.

Specimen examined: INDIA. Tamil Nadu: Coimbatore District: Agriculture College Estate, 467 m, 22.06.1957, K. Subramanyam 3538 (MH).

35. Eragrostis tef (Zucc.) Trotter, Boll. Soc. Bot. Ital. 1918(1): 62. 1918.

Poa tef Zucc., Diss. Ditef s.p. 1775. Type: AFRICA, Ethiopia; cultivated at Florence from seeds collected by Bruce in Ethiopia, Bruce s.n. (holo FI, n.v.).

(Fig. 9 \& Plate 4)

Perennial. Culms 30-100 cm high, erect, loosely tufted; nodes brownish. Leaf sheaths mostly glabrous, mouth bearded. Ligule a tuft of cilia. Leaf blades 3-25 × 0.2$0.4 \mathrm{~cm}$, linear to lanceolate, involute. Panicles $10-45 \times$ $2.5-22 \mathrm{~cm}$, broadly ovate, large and open or contracted; primary branches $4-17 \mathrm{~cm}$ long, alternate, whorled at base, flexible; axils glabrous or hairy. Spikelets $6-8 \times c$.
$1.5 \mathrm{~mm}$, 5-9-flowered, oblong or oblong-lanceolate, olive to leaden grey; florets more or less closely arranged on rachilla, not disarticulating at maturity. Glumes ovate to lanceolate, chartaceous, 1-nerved, 1-keeled, apex acute; lower glume $0.5-1.5 \times c$. $0.4 \mathrm{~mm}$; upper glume $1-2.5 \times$ c. $0.5 \mathrm{~mm}$. Lemmas $1-2.8 \times 0.5-1 \mathrm{~mm}$, ovate to lanceolate, chartaceous, 3-nerved, apex acute. Paleas $1-2.5 \times 0.25-0.5 \mathrm{~mm}$, persistent, elliptic to oblong, 2-nerved, 2-keeled, scaberulous along keels, apex acute to obtuse. Anthers 2, 0.2-0.8 mm long, yellowish. Caryopses 1-1.2 mm long, spindle-shaped, yellowish brown.

Flowering and fruiting: September-December.

Distribution: INDIA: Kerala, Madhya Pradesh, Maharashtra, Rajasthan, Tamil Nadu, Uttarakhand and Uttar Pradesh; Originated in NORTHERN ETHOPIA, introduced in SOUTH AFRICA.

Habitat: Occasional on roadsides, and banks of backwaters in drier areas.

Chromosome nos.: $2 \mathrm{n}=40$ (Moffett \& Hurcombe, 1949), $2 \mathrm{n}=70,80$ (Pienaar, 1955).

Specimens examined: INDIA. Tamil Nadu: Krishnagiri District: Mathigiri, Hosur, 30.12.1916, s.coll. 13983 $(\mathrm{MH})$.

36. Eragrostis tenella (L.) P. Beauv. ex Roem. \& Schult., Syst. Veg., ed. 2: 576. 1817.

Poa tenella L., Sp. Pl.: 69. 1753. Lectotype: INDIA, Herb. Linn. No. 87.33 (LINN), designated by Mitra \& Jain in Manilal, Bot. Hist. Hort. Malabaricus: 151. 1980.

Poa amabilis L., Sp. Pl.: 68. 1753. Eragrostis amabilis (L.) Wight \& Arn., Cat. Indian Pl. 2: 105, n. 1777. 1834. Lectotype: INDIA: Herb. Hermann 2: 59, No. 46 (BM [BM-000621703, image!]), designated by Veldkamp in Cafferty \& al., Taxon 49: 254. 2000.

Poa plumosa Retz., Observ. Bot. 4: 20. 1786-1787. Eragrostis plumosa (Retz.) Link, Hort. Berol. 1: 192. 1827. Eragrostis tenella (L.) P. Beauv. var. plumosa (Retz.) Stapf in Hook.f., Fl. Brit. India 7: 315. 1896. Type: INDIA, Tranquebar, Koenig s.n. in Herb. Retzius 378 (holo LD image!, frag. K000643376, image!).

Eragrostis tenella (L.) P. Beauv. var. breviculmis Stapf in Hook.f., Fl. Brit. India 7: 316. 1896.

Eragrostis amabilis (L.) Wight \& Arn. var. peramangalamensis P. Umam. \& P. Daniel in J. Econ. Taxon. Bot. 22(1): 211. 1998, syn. nov. Type: INDIA, Tamil Nadu, Tiruchirapalli District, Peramangalam, 
along sandy river banks, 26 November 1978, K.M. Matthew, V.S. Manickam \& C. Manoharan RHT 19276 (holo K [K image!; photo in MH, iso RHT!).

Annual. Culms 5-65 cm high, erect or decumbent, geniculate, slender; nodes brownish; internodes 1-7 $\mathrm{cm}$ long with or without punctate glands close below nodes. Leaf sheaths $3-5 \mathrm{~cm}$ long with tubercle based hairs, ciliate along one margin, mouth bearded. Ligule a fringe of short cilia. Leaf blades 2.5-12.5 $\times 0.2-0.5$ $\mathrm{cm}$, flat to involute, lower surface glabrous, upper surface scaberulous to sparsely ciliate. Panicles 15-21 $\times 4-8 \mathrm{~cm}$, open to narrowly ovate to narrowly spicifrm or contracted; primary branches up to $5 \mathrm{~cm}$ long, alternate, with or without irregular glandular areas; axils ciliate; pedicels $c .3 \mathrm{~mm}$ long, slender. Spikelets 2-3 $\times 0.8-1 \mathrm{~mm}, 4-10$-flowered, ovate to lanceolate or oblong, green to greenish purple; rachilla slender, zigzag; florets more or less loosely arranged on rachilla, disarticulating from above downwards. Glumes ovate to lanceolate, chartaceous, 1-nerved, margins entire or minutely serrulate, 1-keeled, scabrous along keel, apex acute to subacute; lower glume $0.4-0.6 \times 0.2-0.3 \mathrm{~mm}$; upper glume $0.5-1 \times 0.2-0.4 \mathrm{~mm}$. Lemmas $0.6-0.8 \times$ $0.3-0.4 \mathrm{~mm}$, ovate to lanceolate or elliptic, chartaceous, 3-nerved, 1-keeled, scabrid along keel, apex acute to obliquely truncate. Paleas $0.6-0.7 \times 0.2-0.3 \mathrm{~mm}$, caducous, elliptic to oblanceolate, chartaceous, 2nerved, 2-keeled, ciliate along keels, apex acute to obtuse; cilia on keels of palea stiff, bulbous based and equal to or longer than the width of palea body; palea flaps almost equal in width to its body. Anthers 3, 0.2$0.3 \mathrm{~mm}$ long, purplish. Caryopses $0.3-0.5 \times 0.2-0.25$ $\mathrm{mm}$, ovoid to ellipsoid, light brown.

Key to the varieties

1a. Panicles lax; branches over $1 \mathrm{~cm}$ long, spreading ...36.1. var. tenella

1b. Panicles contracted; branches less than $1 \mathrm{~cm}$ long, appressed $\quad$...36.2. var. insularis

36.1. Eragrostis tenella (L.) P. Beauv. var. tenella.

(Fig. 9 \& Plate 4)

Panicles lax; branches 1-5 cm long, spreading.

Flowering and fruiting: June-December; stray flowering almost throughout the year.

Distribution: INDIA: Almost throughout; Tropical parts of the world.

Habitat: Grows in disturbed fields such as roadsides, bunds of paddy fields, waste places, riversides, seashores, plantations, agricultural fields and hills.
Chromosome nos.: $2 \mathrm{n}=20$ (Chen \& Hsu, 1962; Larsen, 1963; Tateoka, 1965), $2 \mathrm{n}=60$ (Christopher \& Abraham, 1974).

Vernacular names: Sanna purlai hullu (Kannada); Chinna garikai gaddi (Telugu).

Notes: This is a highly variable species with panicle of dissimilar appearance. The culms are slender and panicles are open to plumose in one group. Here the panicle branches and pedicels are capillary and spikelets are pale green. Another group of variants have more or less stout culms and somewhat spiciform panicle. Here the spikelets are purplish green. The latter variants have close morphological similarities with forms of E. viscosa (Retz.) Trin. with spiciform panicle. Therefore, many collections of E. tenella in the herbaria have been wrongly determined as E. viscosa. Unlike the latter, E. tenella has comparatively smaller spikelets and longer and bulbous based cilia on palea keels (Vivek \& al., 2019).

Umamaheshwari \& P. Daniel (1998) recognized a variety, E. amabilis var. peramangalamensis based on the differences like glabrous axils of panicle branches, slightly smaller spikelets and truncate lemma apex. During the present study it was found that these are variable characters found in various forms of the species and as such this variety is treated here as a synonym of E. tenella var. tenella.

Poa amabilis L. (1753) and Poa tenella L. (1753) are taxonomic synonyms published at the same time. Nicolson \& al. (1988) point out that it was Munro (1862) who merged these two names for the first time choosing the name $P$. amabilis $\mathrm{L}$. for the combined taxon and accepted Eragrostis amabilis as the correct name. Subsequently, these two names have been invariably applied to the taxon in Indian flora. However, according to Peterson \& al. (2018), the name Eragrostis tenella should be accepted for the taxon.

Specimens examined: INDIA. Andaman Islands: Little Andaman: Dugong Creek, 04.09.1976, N. Bhargava 4393 (CAL); Forest Colony Campus, 04.01.1976, $N$. Bhargava 3295 (CAL); Middle Andaman: Mayabunder, 01.08.1974, N. Bhargava 1985 (CAL); Nicobar: Car Nicobar, Malaca, sea level, 11.08.1973, N.P. Balakrishnan $444(\mathrm{MH})$; Great Nicobar, Copenheat, 21.07.1976, N.P. Balakrishnan 3929 (CAL); North Andamans: Katohal Islands, 24.04.1977, $P$. Chakraborty 5538 (CAL); Narcondum Island, Near Police Campus, 22.09.1977, N.C. Nair 6205 (CAL); South Andaman: Havelock Islands, 2m, 20.01.1959, 
K. Thothathri 9096 (MH); Port Blair, Around garden, \pm 20 m, 15.09.1973, s.coll. 357 (MH). Andhra Pradesh: Ananthapuramu District: S.K. University Campus, 350 $\mathrm{m}, 16.06 .1981$, T. Pulliah \& N. Yesoda $160(\mathrm{MH})$; Chittoor District: Chittoor, 09.10.1902, s.coll. 5503 (MH); Mammandur Valley, 12.10.1958, K. Subramanyam 6905 (MH); Godavari District: Annavaram, 20.01.1916, s.coll. 12620 (MH); Dowleshwaram, 01.05.1902, C.A. Barber 4359 (MH); 18.05.1902 \& 4364 (MH); Dwarapudi, 03.04.1902, C.A. Barber 4340 (MH); Samalkota, 24.08.1902, C.A. Barber 4491 (MH); Godavari District: Ankanna Palem, s.dat., D.C.S. Raju 209 (CAL); Kovvur, 17.04.1902, C.A. Barber 4340 (MH); Palagudem, 08.10.1930, $V$. Narayanaswami 4487 ( $\mathrm{MH})$; Guntur District: Kondamedu Fort, 18.10.1902, C.A. Barber 4712 (MH); Hyderabad District: Katasintha, 28.06.1958, K.M. Sebastine 6008 (CAL \& MH); Phringipuram, 14.10.1902, C.A. Barber 4605 (MH); Krishna District: Chilakalapudi, 23.11.1984, P. Venkanna 5397 (MH); Collapudi, 11.08.1984, P. Venkanna 5268 (MH); Menginapudi, 03.08.1984, P. Venkanna 5135 (MH); Thukkulur, 12.09.1985, P. Venkanna 5738 (MH); Kurnool District: Diguvametta, 440 m, 06.08.1972, J.L. Ellis 42153 (MH); Nallamalai, on the way to Bairanii, 325 m, 17.11.1969, J.L. Ellis 32478 (CAL \& MH); Nalconda District: Vijayapuri, 110 m, 17.12.1959, K.M. Sebastine 9847 (CAL \& MH); Visakhapatanam District: 24.12.1969, G.V. Subbarao 32789 (CAL); Araku Valley, 16.09.1961, N.P. Balakrishnan 621 (CAL); Chilakala Gidda, 100 m, 24.12.1969, G.V. Subbarao 32789 (MH); Warangal District: Pakhal R.F., 290 m, 28.11.1960, K.M. Sebastine 11667 (CAL \& MH). Arunachal Pradesh: Ziro District: Yacholi-Subansiri F.D. (NEFA), 28.09.1959, G. Panigrahi 19768 (CAL). Assam: Dima Hasao District: Haflong-North Cachar Hills, 18.08.1908, William G. Craib 298 (CAL). Bihar: Champaran District: Nolaipur Forest, 09.11.1963, B.V. Shetty 138 (CAL); Manguraha Forest, 14.04.1963, K. Thothathri 10089 (CAL); Raxaul, 08.08.1964, S.P. Banerjee 26 (CAL); Nalanda District: Banglapur, 10.11.1960, P.C. Nanda 2233 (CAL). Chhattisgarh: Bastar District: Abujh Marh, 20.05. 1983, G.P. Roy 35218 (CAL); Keskal, 667 m, 16.11.1958, K. Subramanyam 7109 (CAL \& MH). Daman: 26.09.1963, M.Y. Ansari 93667 (CAL). Gujarat: Anand District: Vallabh-Vidyanagar, 27.09.1967, G.L. Shah 14135 (CAL). Jammu \& Kashmir: Pulwama District: Bargam, 23.09.1978, B.V. Shetty 6694 (CAL). Jharkhand: Hazaribagh District: 04.10.1883, C.B. Clarke 33813 (CAL); Sahibganj District: Rajmahal,.10.1894, Mokim
1502 (CAL). Karnataka: Bangalore District: Bangalore, Jan. 1902, C.A. Barber 4811 (MH); Bellari District: Hagai, 19.09.1916, s.coll. 13759 (MH); Hagari, 10.02.1917, s.coll. 14287 (MH); Hampi, 14.10.1919, s.coll. $15979(\mathrm{MH})$; Coorg District: Fraserpet, 06.06.1902, C.A. Barber 4404 (MH); Mysore District: Mysore: s.dat., G. Thomson s.n., MH Acc. No. 86119 (MH); North Kanara District:.Oct. 1890, W.A. Talbot 2383 (CAL); Uttara Kannada District: Mangalore, Jan. 1902, C.A. Barber 4816 (MH). Kerala: Kannur District: Muzhuppilangad, $\pm 50,16.12 .1979$, V.S. Ramachandran 65263 (MH); Nileshwar, 175 m, 29.01.1979, V.J. Nair 59985 (MH); Kasaragod District: Nileshwar, 06.12.1919, s.coll. 6291 (MH); Kozhikode District: Malabar Botanical Garden, 18.10.1999, P.M. Krishnan 956 (Herbarium of Malabar Botanical Garden \& Institute for Plant Sciences, Kozhikode, Kerala); Palakkad District: Palghat, 19.12.1919, s.coll. 16318 (MH); Trissur District: Chavakkad seashore, sea level, 06.09.1976, K. Ramamurthy 47670 (MH). Lakshadweep: Kavarathi Island: 04.12.1960, B.M. Wadhwa 68669 (CAL). Madhya Pradesh: Gwalior District: Lafa, 28.10.1970, G. Panigrahi 13030 (CAL); Pagara Dam, 13.11.1962, G. Panigrahi 5795 (CAL); Hoshangabad District: Hoshangabad, $300 \mathrm{~m}$, 28.09.1960, J. Joseph 11091 (CAL \& MH); Indore District: Khurel Village, 24..10.1962, A.S. Rao 84157 (CAL). Saugor District: Tigorda- Hirapur R.F., 425 m, 01.11.1960, N.P. Balakrishnan 11445 (CAL \& MH). Maharashtra: Buldhana District: Sultanpur, 23.09.1982, P.G. Diwakar 164090 (BSI); Pune District: LonavlaNear Valuan Dam, 18.08.1964, B. Venkata Reddi 98626 (BSI); Poona, 14.05.1956, Puri 2838 (CAL); Shivneri Hills, 12.10.1962, M.Y. Ansari 83725 (CAL). Odisha: Cuttak District: Angul, 07.01.1962, J.S. Singh 1557 (CAL); Baludi, 03.08.1919, N. Annandale 1204 (CAL); Jagatpur, 22.05. 1967, M.K. Ghosh 228 (CAL); Mahanadi Shores, 15.05. 1968, M.K. Ghosh 1977 (CAL). Rajasthan: Banswara District: Bagaycha, 22.10.1976, V. Singh 3773 (CAL); Barmer District: 17.10.1976, B. V. Shetty 2231 (CAL); 14.11.1981, R.P. Pandey 7987 (CAL); Ganganagar District: Padampur, 29.10.1959, G. Panigrahi 20570 (CAL); Thakari, 12.11.1976, G.P. Roy 3852 (CAL); Churu District: 21.11.1976, G.P. Roy 4005 (CAL); Jaipur District: 19.08.1964, B.M. Wadhwa 4829 (CAL); Mutha, 15.02.1965, B.M. Wadhwa 8149 (CAL); Jhunjhunu District: Pilaui, Nov. 1961, B.D. Deshpande 01 (BSD); Jodhpur District: 14.09.1973, G.L. Tiwari 610 (CAL); Sirohi District: Erinpura, 01.10.1960, Seshagiri Rao Rolla 66752 (CAL). Sikkim: West Sikkim District: 
Chakung, 14.11.1981, B. Krishna 1787 (BSHC); Gayzing, 19.08.1985, D.C.S. Raju 4469 (BSHC); Melli Reserve Forest, 23.02.1986, B.K. Shukla 18782 (BSHC); 26.07.1986, A.K. Verma 6716 (BSHC). Tamil nadu: Chengalpetu District: Kuridimalai foot, $600 \mathrm{~m}$, 27.08.1956, K. Subramanyam 620 ( CAL \& MH); Poonamalli, 17.09.1917, s.coll. 14849 (MH); Sanjivi Hills, 14.02.1957, K.M. Sebastine 2304 (CAL); Chennai District: Madras, 1887, J.S. Gamble s.n., MH Acc. No. 58095 (MH); Coimbatore District: Bharathiar University Campus, 26.05. 2010, C.P. Vivek 126114 (MH); BSI Campus, 27.04.2010, C.P. Vivek 126103 (MH); Burliar, 29.04.2010, C.P. Vivek 126108 (MH); Ibid.,15.05.2010, C.P. Vivek 126112 (MH); Coimbatore, 14.02.1957, K.M. Sebastine 2304 (MH); Coimbatore, 18.11.1964, K.N. Subramanyan 1754 (FRC); Jalavadi, $\pm 762 \mathrm{~m}, 06.03 .1931$, K.C. Jacob 50 (MH); Kunjur, \pm 853 m, 27.01.1931, S.R. Raju \& Naganathan 4996 (MH); Girgagandy Palur, 19.07.1968, K.N. Subramanyan 3488 (FRC); Kovalamthittu, Pichavaram R.F, Vridhachalam Range, 04.03.1970, K.N. Subramanyan 3911 (FRC); Kuridimalai-Foot, 600 m, 27.08.1956, K. Subramanyam $620(\mathrm{MH})$; Madeshwaram Malai-slope, $\pm 1066 \mathrm{~m}$, 09.02.1930, V. Narayanaswami 19819 (MH); Maruthamalai, 654 m, 29.12.1956, K.M. Sebastine 1866 (MH); 27.06.2010, C.P. Vivek 126124 (MH); Siruvani, 03.07.2010, C.P. Vivek 126126 (MH); T.N.A.U. Paddy field, 23.06.2010, C.P. Vivek 126118 (MH); Erode District: Bhavani Sagar, 03.10.1987, N. Venkatasubramanyan 719 (FRC); Chittar, 15.08.1988, N. Venkatasubramanyan 1642 (FRC); Kanchipuram District: Vedanthangal Wild Life Sanctuary, $\pm 120 \mathrm{~m}$, 25.01.1976, A.N. Henry 47025 (MH); Madurai District: Forbay Dam, near-Kumili, 22.06.1959, K. Subramanyam 8122 (CAL); Kodaikkanal, 19.12.1905, C.A. Barber 7472 (MH); Nilgiri District: Aug. 1886, J.S. Gamble 17884 (CAL); Anaikkatti-Kedar Halla, 850 m, 21.08.1970, G.V. Subbarao 36150 (MH); Anthikkadavu Forest, 525 m, 31.07.1975, E. Vajravelu 46392 (MH); Pudukkottai District: Viralimalai, \pm 130 $\mathrm{m}, 11.08 .1984, \quad$ C. Arulappan $143 \quad(\mathrm{MH})$; Ramanadapuram District: Krusadi Island, 11.05. 1949, D. Daniel Sundaraj \& J. Sakharam Rao s.n. (MH); Pamban, 29.01.1945, D. Daniel \& S.R. Raju 20080 $(\mathrm{MH})$; Pamban, $\pm 10 \mathrm{~m}, \quad 20.12 .1986, \quad V$. Balasubramanyam 1009 (MH); Perunazhi, $\pm 75 \mathrm{~m}$, 14.01.1989, V. Balasubramanyam $2056(\mathrm{MH})$; Ramanathapuram, $50 \mathrm{~m}, 21.12 .1986, \quad V$. Balasubramanyam $1024(\mathrm{MH})$; Sethukarai, $\pm 50 \mathrm{~m}$, 23.12.1986, V. Balasubramanyam 1150 (CAL \& MH); Salem District: Hogainakkal-riverbank, 275 m,
11.07.1964, E. Vajravelu 20543 (MH); Pennagaram R.F., 275 m, 02.12.1964, E. Vajravelu 22404 (MH); Tanjavur District: Kodiakkarai R.F., Sea level, 02.02.1987, S. Ragupathy 151 (MH); Tinnevelly District (Tirunelveli): Chinnathadagam, therkkumaai, Way to Nupura Ganga, 167 m, 11.06.1957, K. Subramanyam 3385 (MH); Kingh Manimuthar, 300 m, 26.06.1957, K.M. Sebastine 3663 (MH); Oodatham Estate, Courtallam, 400 m, 26.07.1957, K. Subramanyam 3844 (MH); Mundanthorai Ghat, 14.05.1901, C.A. Barber 2750 (MH); Therkkumalai, Courtallam, $400 \mathrm{~m}$, 24.07.1957, K. Subramanyam 3774 (CAL \& MH); Vadugapatti, 27.04.2010, C.P. Vivek 126105; 126106 (MH). Tripura: Agartala, 26.09.14, P.M. Dobbarman 56 (CAL). Uttarakhand: Kumaon District: Bageshwar, 17.09.1957, T.A. Rao 4008 (BSD). Uttar Pradesh: Ghaziabad District: 04.03.1959, T.A. Rao 8264 (BSD); Gonda District: Janakpur, 30.05.1986, K.K. Khanna \& R. Saran 37295 (CAL). West bengal: Bankwara District: Bilaspur, 12.11.1981, M.N. Sanyal 1461 (CAL); Birbhum District: Hambazar, 27.04.1966, R.K. Basak 279 (CAL); Burdwan District: Burakar, 07.12.1874, C.B. Clarke 25249 (CAL); Burdwan, 14.10.1894, J.D. Hooker 1183 (CAL); Dishirgarh, 25.09.1972, R.N. Banerjee 106 (CAL); Krishna Sagar, Burdwan Town, 28.11.1966, D.K. Banerjee 4826 (CAL); Sanctoria, 22.09.1972, R.N. Banerjee 53 \& 57 (CAL); Dinajpur District: Atreyee river bank, 28.10.1983, R.N. Banerjee \& M.C. Biswas 16060 (CAL); Balurghat, 08.04.1984, R.N. Banerjee 16271 (CAL); 23.10.1961, D.K. Banerjee 48 (CAL); Raigunj, 20.08.1984, R.N. Banerjee 17598 (CAL); Howrah District: Botanic Garden, Central Part, 27.05. 1967, D.K. Banerjee 4857 (CAL); Dakshinmanju, 11.05. 1964, S.S.R. Bennet 705 (CAL); Hoogly, Chapadaqa, 03.12.1967, Subire Sen 101 (CAL); Howrah, 15.09.1894, J.D. Hooker 1224; Ibid., 17.09.1894, J.D. Hooker 1205 (CAL); Malda District: Malda, 29.04.1966, R.M. Dutta 269; Manikchowk, 21.08.1966, R.M. Dutta 405 (CAL); Midnapore District: Chatra, 05.12.1975, Sudarsan Maji 2496 (CAL); Midnapore Coast, 25.02.1965, T.A. Rao 4042 (CAL); Pathrisole, 18.12.1955, M.J. Ganguly (CAL); Murshidabad District: Murshidabad, s.dat., D.N. Guhabakshi 260 (CAL); Parganas District: Garia, 06.08.1961, G. Sengupta 45 (CAL); Purulia District: Matha, near a spring, 13.09.1965, K.C. Malik 287 (CAL); Nutandi, Kuilapal, 16.02.1970, K.C. Malik 972 (CAL).

36.2. Eragrostis tenella var. insularis C.E. Hubb. in Bull. Misc. Inform. Kew 1939: 654. 1939. Type: MAURITIUS, Cane fields near Moka, April 1939, Vaughan 1837 (holo K, photo in MH!). 


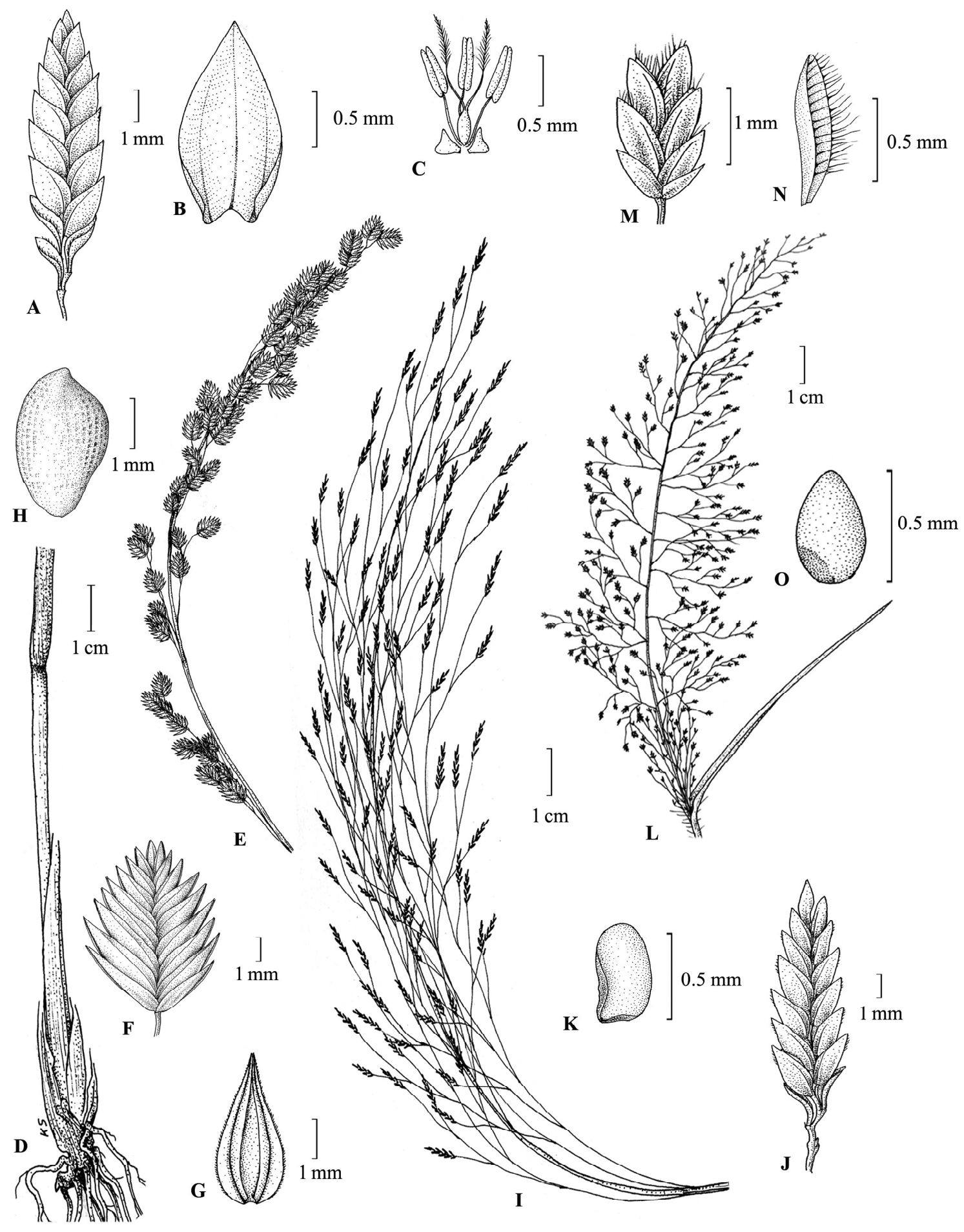

Fig. 9. Eragrostis subsecunda (Lam.) E. Fourn. (A-C). A. Spikelet; B. Lemma-ventral view; C. Lodicules, Stamens \& Pistil. E. superba Peyr. (D-H). D. Culm; E. Inflorescence; F. Spikelet; G. Palea-ventral view; H. Caryopsis. E. tef (Zucc.) Trotter (I-K). I. Inflorescence; J. Spikelet; K. Caryopsis. E. tenella (L.) P. Beauv. var. tenella (L-O). L. Inflorescence; M. Spikelet; N. Palealateral view; O. Caryopsis. 
Panicles contracted; branches less than $1 \mathrm{~cm}$ long, appressed to main rachis.

Flowering and fruiting: Almost throughout the year.

Distribution: INDIA: Tamil Nadu and West Bengal; MADAGASKAR, MASCARENE ISLANDS and SRI LANKA.

Habitat: Coastal sandy seashores.

Chromosome nos.: $2 \mathrm{n}=40,60$ (Gould \& Soderstrom, 1974).

Notes: This is a coastal taxon, distinct from other forms of E. tenella var. tenella by its linear panicle with longer and appressed branches.

Specimens examined: INDIA. Tamil Nadu: Chengalpetu District: Thirukazhukudram, $50 \mathrm{~m}$, 04.09.92, S. Krishnan, N.P. Samson \& P. Ravichandran 273 (MH); Kancheepuram District: 2012, C.P. Vivek 126161 (MH); Ramanathapuram District: Sayalgudi, Kannirajapuram, \pm 75 m, 25.12.1989, $\quad V$. Balasubramaniam 2310 (MH); Uttarakosamangai, 01 Jan. 1958, V. Balasubramaniam 1516 (MH); Thanjavur District: Point Calimere, Sea level, 23.01.1961, J.L. Ellis $11835(\mathrm{MH})$.

37. Eragrostis tenuifolia (A. Rich.) Hochst. ex Steud., Syn. Pl. Glumac. 1: 268. 1854.

Poa tenuifolia A. Rich., Tent. Fl. Abyss. 2: 425. 18501851. Lectotype: ETHIOPIA, in locis incultis Vallium prope Adoam, 18 September 1837, Schimper 92 (P), designated by S.M. Phillips, Fl. Ethiopia 7: 122. 1995.

(Fig. 10 \& Plate 3)

Perennial. Culms $12-85 \mathrm{~cm}$ high, erect to decumbent, geniculate at lower portion; nodes brownish; internodes $4-12 \mathrm{~cm}$ long. Leaf sheaths $4-8 \mathrm{~cm}$ long, sparsely ciliate along one margin, mouth bearded. Ligule a fringe of cilia. Leaf blades $9-46 \times 0.2-0.5 \mathrm{~cm}$, linear to lanceolate, upper surface scabrid to sparsely ciliate. Panicles 5-11 $\times 2.5-6 \mathrm{~cm}$, broadly ovate, open; primary branches alternate; axils ciliate; pedicels 3-7 $\mathrm{mm}$ long, glandular. Spikelets 4-10 × 1.8-2 mm, 6-13-flowered, lanceolate to oblong, serrate in appearance, green to blackish or grey; rachilla zigzag; florets more or less closely arranged on rachilla, disarticulating from below upwards. Glumes ovate to oblong, chartaceous, often nerveless or nerve obscure, apex acute to obtuse; lower glume $0.4-0.9 \times 0.2-0.4 \mathrm{~mm}$; upper glume $0.8-1 \times 0.4-$ $0.6 \mathrm{~mm}$. Lemmas $1.5-2 \times 0.9-1 \mathrm{~mm}$, ovate to oblong or elliptic, chartaceous, 3-nerved, 1-keeled, often glabrous, at times scabrid towards apex, apex acute to subacute or lobed. Paleas $1.5-1.7 \times 0.4-0.6 \mathrm{~mm}$, persistent, elliptic, chartaceous, 2-nerved, 2-keeled, scaberulous along keels, apex obtuse to truncate; palea flaps almost equal in width to its body. Anthers 3, 0.6-0.9 mm long, cream-coloured. Caryopses 1-1.2 $\times$ c. $0.6 \mathrm{~mm}$, ellipsoid to oblongoid, ventrally flattened to grooved, truncate at ends, deep reddish.

Flowering and fruiting: March-October.

Distribution: INDIA: Almost throughout; Tropical parts of the countries; AFRICA, AUSTRALIA, MYANMAR and PAPUA NEW GUINEA.

Habitat: Usually grows in higher elevations, in open dry places, on roadsides, in wetlands, rare in grasslands, fallow grounds, weeds in plantations.

Vernacular name: Kadu sanna samai hullu (Kannada).

Chromosome no.: 2n = 40 (Tateoka, 1965).

Notes: This is commonly found associated with E. nigra in hilly areas and can be easily recognized from other species of Eragrostis by the unique appearance of serrate margins of spikelets with caryopses that often exposed.

Specimens examined: INDIA. Andhra Pradesh: Chittoor District: Irumalai Hills, \pm 792 m, 12.10.1938, K.C. Jacob 475 (MH); Visakhapatnam District: Ananthagiri - West side Bunglow, 910 m, 11.05.1964, G.V. Subbarao 19470 (MH); Arabi Valley, 08.09.1952, Daniel Sunder Raj 21375 (MH). Karnataka: Bangalore, Feb. 1908, A. Meebold 8188 (CAL); Bellary District: Ramandrug, 09.10.1919, s.coll.15942 (MH). Kerala: Idukki District: Kulamavu, 850 m, 25.09.1981, C.N. Mohanan \& Ramanujan 71966 (CAL \& MH); Meenmutty, 600 m, 28.05.1982, C.N. Mohanan 74088 (MH); Munnar, 30.05.1944, K.C. Jacob 20483 (MH); Kannur District: Tolpetty, 840 m, 06.07.1978, s.coll. 54153 (CAL, MH); Begur, 825 m, 03.03.1979, V.S. Ramachandran 62035 (MH); Brahmagiri, $950 \mathrm{~m}$, 04.03.1979, s.coll. $62068(\mathrm{MH})$; Bavel, $825 \mathrm{~m}$, 10.08.1979, V.S. Ramachandran 62796 (CAL, MH); Kottayam District: Kurisumala-Changanasseri, 03.05.1984, V.T. Antony 467 (MH); Palakkad District: Aruvampara - On the way, $850 \mathrm{~m}, 08.10 .1979$, N.C. Nair 64432 (MH); Thiruvanandapuram District: Ponmudi, 1300 m, 25.05.1979, M. Mohanan 63248 (MH); Wayanad District: Meenangadi, 18.07.2010, C.P. Vivek $126134(\mathrm{MH})$. Madhya Pradesh: Damoh District: Bandakpur, 19.08.1978, B.K. Shukla 28744 (CAL \& BSA); Singhrampus, 16.09.1979, B.K. Shukla 29953 (CAL); Ujjain District: Makla, 16.10.1908, J.H. Burkill 31315 (CAL). Maharashtra: Junnar District: 

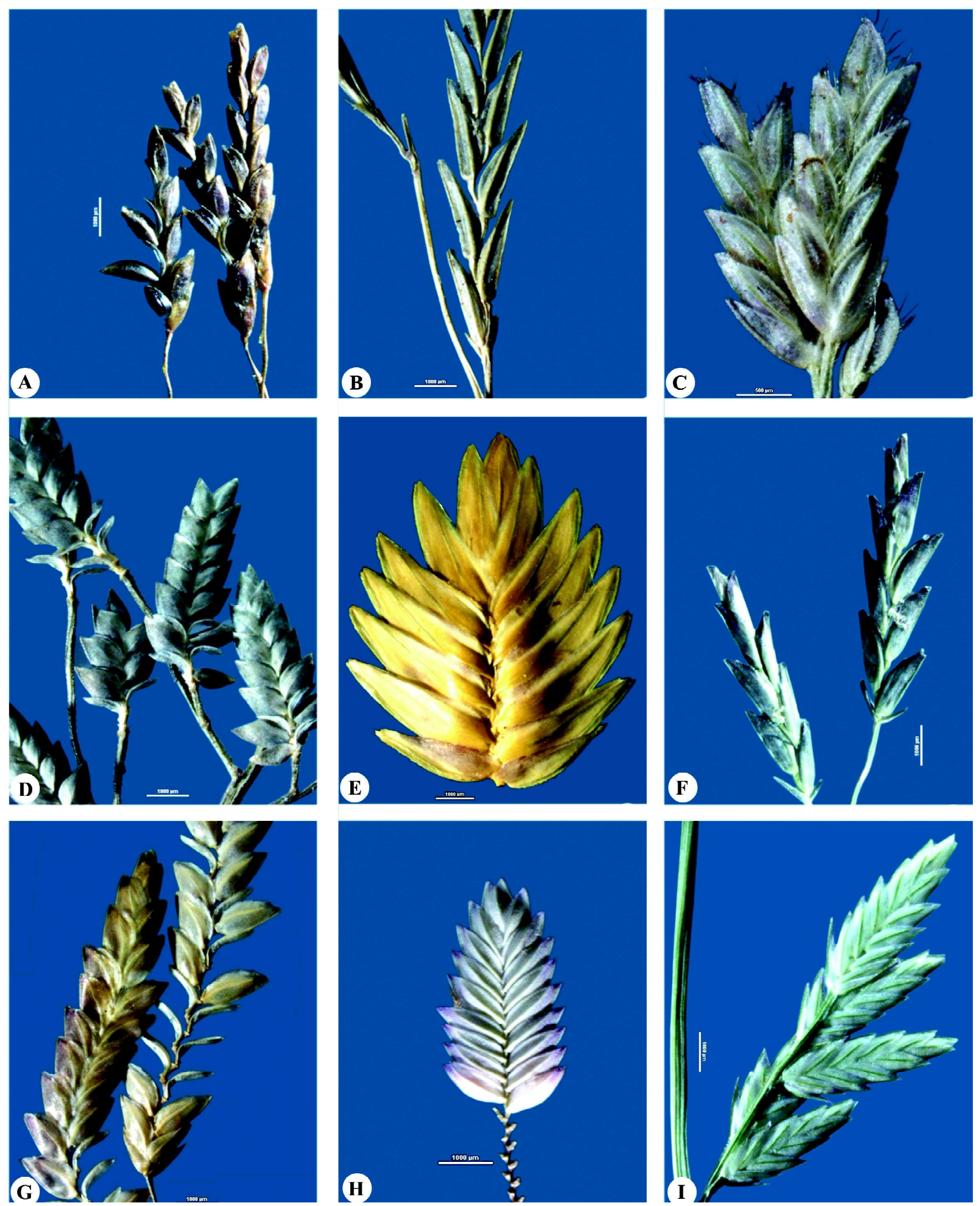

Plate 3. Spikelets of : A. Eragrostis papposa (Roem. \& Schult.) Duf. ex Steud.; B. E. plana Nees; C. E. riparia (Willd.) Nees; D. E. schweinfurthii Chiov.; E. E. superba Peyr.; F. E. tenuifolia (A. Rich.) Hochst. ex Steud.; G. E. tremula Hochst. ex Steud. var. tremula; H. E. unioloides (Retz.) Nees ex Steud.; I. E. zeylanica Nees \& Mey. 
Bhivardikhurd, 30.09.1965, K. Hemadri 107519 (CAL); Pimparwadi, 05.10.1965, K. Hemadri, 106822 (CAL); Yenere, 08.10.1965, Hemadri, K. 106925 (CAL). Tamil Nadu: Coimbatore District: Hasanur, $\pm 914 \mathrm{~m}$, 22.06.1929, K.C. Jacob 18564 (MH); Kallar, 29 Apr. 2010, C.P. Vivek 126109 (MH); Kottadi, \pm 1066 m, 11.03.1931, 275 (MH); Palamalai, 17.09.1969, M.V. Viswanathan 168 (MH); Perinochikotta, $\pm 914 \mathrm{~m}$, 06.02.1930, V. Narayanaswami 19549 (MH); Ramaswami Koil, \pm 1097 m, 24.01.1931, S.R. Raju \& Naganathan 4901 (MH); Shiruvani, 866 m, 07.12.1956, K. Subramanyam 1653 (CAL \& MH); Dindigul District: Kodaikanal Ghat, 09.07.1898, s.coll. s.n. (MH); Kodaikanal Hills, 19.12.1905, C.A. Barber 7460 (MH); Erode District: Minchguli Valley, Sathy Taluk, 02.08.1987, N. Venkatasubramanyan 599 (FRC); Madurai District: Hosur-Central Cattle Farm, 08.10.1926, K.C. Jacob 16940 (MH); Kumili, 850 m, 25.06.1959, K. Subramanyam 8208 (MH); Nilgiri District: Coonoor, along railway track, $1700 \mathrm{~m}$, 22.10.2011, C.P. Vivek 126156 (MH); Hulical Droog, 1733 m, 27.08.1957, s.coll. 4137 (MH); Kotagiri, April 1915, L.J. Sedwick 651 (CAL); June 1916, L.J. Sedgwick 1413 (CAL); Kunnia Combai R.F., 2000 m, 01.09.1957, K.M. Sebastine 3991 (MH); Ootacamund, 09.01.1902, s.coll. 4160 (MH); Ootacamund, 26.08.1901, C.A. Barber 3462 (MH); Reserve Forest near Abayaranyam rest house, 15.05.63, K.N. Subramanyan 670 (FRC); Salem District: Metigiri, 12.12.1917, s.coll. 15436 (CAL \& MH); Pulluhalli, 26.12.1913, s.coll. 9861 (MH); Yercaud-Bauxite Hills, 1470 m, 19.06.1965, A.V.N. Rao 26771 (CAL \& MH); Yercaud-Lady's Seat-Karadu, 1420 m, 27.07.1966, S. Karthikeyan 28295 (CAL \& $\mathrm{MH})$.

38. Eragrostis tremula Hochst. ex Steud., Syn. Pl. Glumac. 1: 269. 1854. Type: Nubia; Hochstetter Hrbr. un. it. no. 6.

Poa tremula Lam., Tabl. Encycl. 1: 185. 1791. Type: "E. Senegal, D. Roussillon. "Africa: Senegal; Roussillon s.n. (P-LAM).

Poa multiflora Roxb., Fl. Ind. 1: 340. 1820, non Forssk. 1775. Type: INDIA, Roxb. s.n.

Eragrostis multiflora Trin., Mém. Acad. Imp. Sci. St.Pétersbourg, Sér. 6, Sci. Math. 1(4): 401. 1830.

Annual. Culms $15-85 \mathrm{~cm}$ high, densely or loosely tufted, geniculate; nodes brownish; internodes $c .6 \mathrm{~cm}$ long. Leaf sheaths $3-4.5 \mathrm{~cm}$ long, sparsely ciliate along one margin, glabrous when mature, mouth bearded. Ligule a fringe of cilia. Leaf blades $3-20 \times 0.2-0.4 \mathrm{~cm}$, linear to lanceolate, upper surface scabrid to tuberclebased ciliate. Panicles 7-30 $\times 5-20 \mathrm{~cm}$, largely open, lax and as long as rest of the plant; primary branches alternate; axils more or less ciliate; pedicels over $5 \mathrm{~mm}$ long, slender, capillary. Spikelets 10-50 × 2-2.3 mm, 10-70-flowered, linear to oblong, narrowly curved, yellowish green to purplish; rachilla slender, zigzag; florets closely arranged on rachilla, disarticulating from below upwards. Glumes unequal, ovate to lanceolate, chartaceous, 1-3-nerved, 1-keeled, glabrous or minutely scabrous along keel, apex acute to sub-acute; lower glume $0.8-1.2 \times 0.4-0.5 \mathrm{~mm}$; upper glume $1.2-$ $1.5 \times 0.9-1 \mathrm{~mm}$. Lemmas $1.5-1.7 \mathrm{~mm}$ long, broadly ovate, chartaceous, 3-nerved, 1-keeled, scabrous along keel, apex acute to subacute or narrowly obtuse. Paleas 1.4-1.5 mm long, persistent, elliptic, 2-nerved, 2keeled, scaberulous along keels above middle, apex acute to obtuse; palea flaps narrower than its body. Anthers 2 or $3, c .0 .1 \mathrm{~mm}$ long, purplish. Caryopses $0.4-0.6 \mathrm{~mm}$ long, ovoid to subglobose or orbicular, yellowish brown.

Key to the varieties

1a. Glumes caducous; upper glume 1-nerved ...38.1. var. tremula

1b. Glumes persistent; upper glume 3-nerved ...38.2. var. gajanandii

38.1. Eragrostis tremula (Lam.) Hochst. ex Steud. var. tremula.

(Fig. 10 \& Plate 3)

Flowering and fruiting: August-December.

Distribution: INDIA: Almost throughout; AFGHANISTAN, MYANMAR, TROPICAL AFRICA.

Habitat: On field margins, roadsides and in wetlands.

Chromosome nos.: $2 \mathrm{n}=20$ (Mulay \& Leelamma, 1956; Tateoka, 1965), $2 \mathrm{n}=30$ (Mulay \& Prasad, 1956), $2 \mathrm{n}=$ 40 (Christopher \& Abraham, 1974).

Vernacular names: Bano, Binnum (Arabic).

Specimens examined: INDIA. Andhra Pradesh: Godavari District: Irumalayaru, 10.12.1902, C.A. Barber 5281 (MH); Kondapudi, 08.12.1902, C.A. Barber 5189 (MH); Peddavegi-Ellore, 09.10.1930, $V$. Narayanaswami 4516 (MH); Kurnool District: Without precise locality, 30.08.1936, K.C. Jacob s.n. (MH); Nellore District: Venkannapalem, 12.03.1962, D.C.S. Raju 246 (CAL). Bihar: Rohtas District: Sasaram, 08.12.1955, B.D. Patil 203 (CAL). Chhattisgarh: Bilaspur District: Bahmui river bed, 03.11.1970, G. Panigrahi 

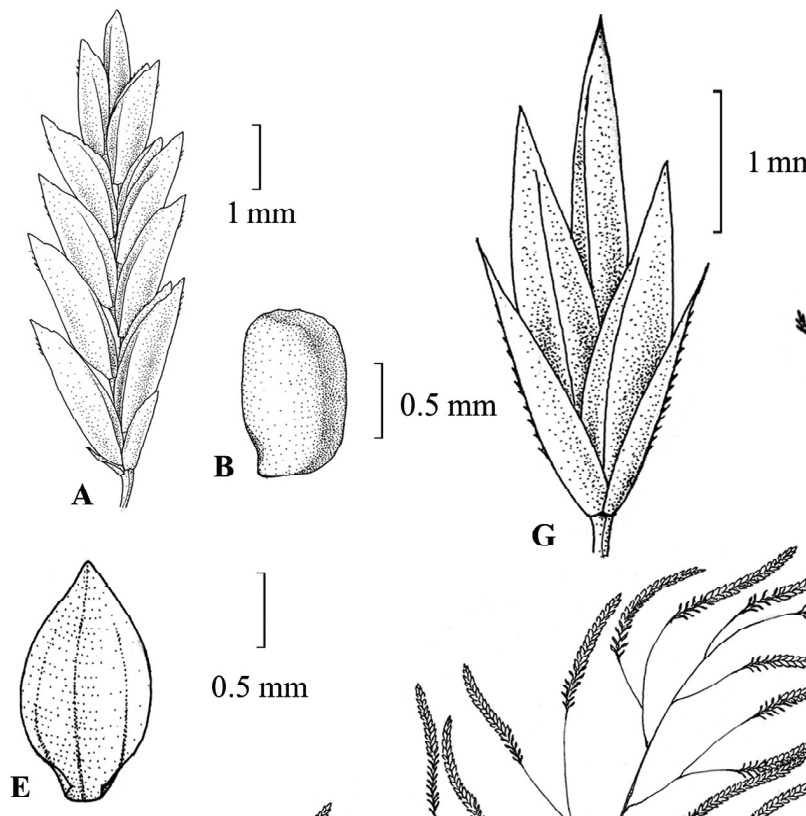

G

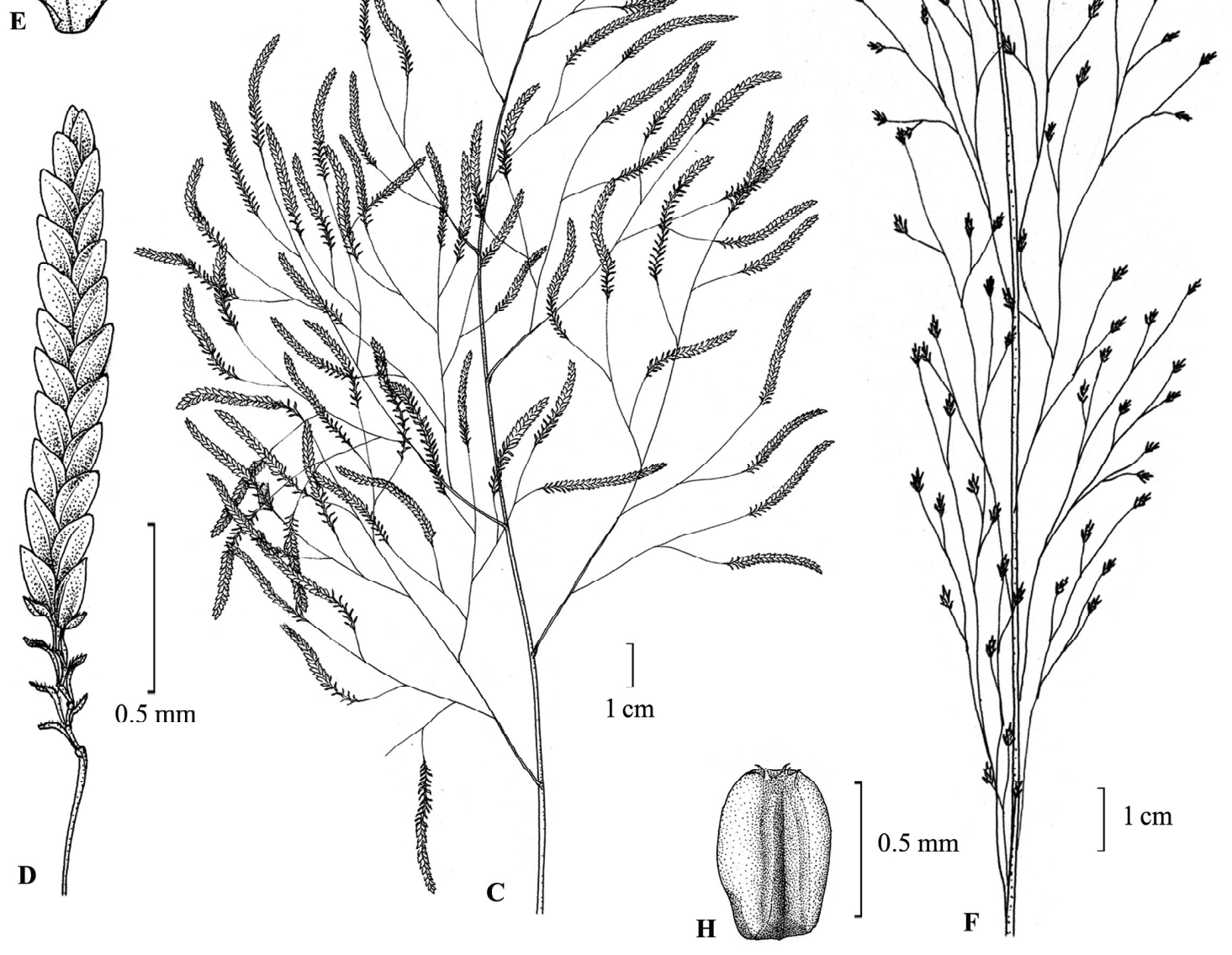

Fig. 10. Eragrostis tenuifolia (A. Rich.) Hochst. ex Steud. (A-B). A. Spikelet; B. Caryopsis. E. tremula Hochst. ex Steud. var. tremula (C-E). C. Inflorescence; D. Spikelet; E. Lemma-ventral view. E. trichodes (Nutt.) Alph. Wood (F-H). F. Inflorescence; G. Spikelet; H. Caryopsis. 
13288 (CAL). Daman and Diu: Daman District: Without precise locality, 24.09.1963, M.Y. Ansari 93515 (CAL). Gujarat: Kachch District: Adesar, 13.09.1968, R.S. Raghavan 114722 (BSI). Haryana: Barahi District: Without preciselocality, 01.03.1977, J.N. Vohra 60216 (BSD); Bhiwani District: Without precise locality, 21.10.1962, N.C. Nair 25067 (BSD); Hisar District: Loudhriwar, 21.10.1962, N.C. Nair 25110 (BSD); Siwani District: 23.10.1967, V.J. Nair 37625 (BSD); Mahendragarh District: Narnaul, 24.10.1962, N.C. Nair 25192 \& 25194 (BSD). Jharkhand: Chota Nagpur Plateau: Chota Nagpur, s.dat., J.J. Wood s.n. (CAL); Ranglur Ghat, 19..10.1883, C. B. Clarke 33902 (CAL); Dumka District: Silingi, 21.12.1957, G. Panigrahi 12027 (CAL); Giridh District: Parasnath, 19..11.1874, C.B. Clarke 24891 (CAL); Ranchi District: Ranchee, 16..10.1873, C.B. Clarke 21242 (CAL); 16..10.1893, s.coll. 21235 (CAL). Karnataka: Eastern Karnataka, Raichur, 15.11.1975, N.P. Singh 141732 (BSI); North Kanara, January 24, W.A. Tabolt 2207 (CAL). Kerala: Kannur District: Muzhuppilangad, 16.12.1979, V.S. Ramachandran 65259 (CAL \& MH); Kasaragod District: Kasaragod, 12.12.1931, G.V. Narayana 6313 (MH); Nileshwar, 08.11.1917 (MH); s.coll. 15315 $(\mathrm{MH}) ; 06.12 .1919$, s.coll. $16310(\mathrm{MH})$. Madhya Pradesh: Gwalior District: Oct. 1889, C. Marier 161 (CAL); Sidhi District: Barka Dol, Sidhi, G. Panigrahi 2170 (CAL). Odisha: Puri District: Puri, 20 Apr. 1965, V. Abraham 316; 315 (CAL); Puri Coast, 02.12.1965, T.A. Rao 5679 (CAL); Puri sandy tarct, 1889, S. Walsh 541961 (CAL). Pujab: Batinda District: 12.05.1963, N.C. Nair 27814 (BSD); Hoshiarpur District: 01.09.1969, U.C. Bhattacharyya 39498 (BSD); Garhshanker, 23.09.1970, O.P. Misra 38245 (BSD). Rajasthan: Churu District: Adsar Village, 18.07.1976, G.P. Roy 2699 (CAL); Barmer District: Dhorimanna $11 \mathrm{~km}$ away, along Gaurka Tala, 29.10.1975, B.V. Shetty, 2350 (CAL); Bikaner District: 25.09.1975, G.P. Roy 2162 (CAL); Bilwara Dist: Badnor, 20.09.78, A.N. Singh 6258 (CAL); Eastern Rajasthan: s.dat., R.B. Majumdar 101 (CAL); Jaipur District: Siran, 25.03.1961, M.A. Rao 14398 (BSD); Jaisalmer District: Mohangarh, 27 Aug.64, B.M. Wadhwa 5054 (CAL); Jalor District: Jalor-Kaniwara near, 13.10.1977, B.L. Vyas 4534 (CAL); Jodhpur District: Agri. Farm, 15.09.1956, S.C. Agarwal 540 (CAL); Chaba, 19.08.1977, A.N. Singh 4328 (CAL); Kharda Randir Village, 25.10.1972, s.coll. 196 (CAL); Shergarh, 08.10.76, A.N. Singh 3129 (CAL); Jhunjhunu District: Pilani, Nov. 1961, B.D. Deshpande 7 (BSD); Pali District: Jawai Dam-Eruinpura near, 01.10.1960, S.R. Rolla 66763 (CAL). Tamil Nadu: Thirunelveli
District: Mundonthorai Ghat, s.dat., C.A. Barber 2773 (MH). Uttarpradesh: Bahraich District: 20.02.1963, C.L. Malhotra 26720 (BSD); Pilibhit District: 04.10.1970, C.L. Malhotra 42543 (BSD); Saharanpur District: Aug. 1984, T.S. Murthy \& A.K. Goel 853 (BSD). West Bengal: Bankuara District: Chakdah, 27.12.79, M.N. Sanyal 1458 (CAL); Birbhum District: Santiniketan, 07.09.1949, K. Biswas 4291 (CAL); 28.01.1956, S.K. Mukherjee 4102 (CAL); Medinipur District: Garbeta range, 18.12.55, J. Ganguly s.n. (CAL); Midnapur Coast, Junput, 24.02.1965, T.A. Rao E4039 (CAL); Purulia District: Kharbani Forest, 07.02.1970, K.C. Malik 729 (CAL).

38.2. Eragrostis tremula var. gajanandii Genda Singh, Bala \& C.S. Purohit in Indian Forester 137 (6): 796. 2011. Type: INDIA, Rajasthan, Jaisalmer District, 07 January 2007, Singh, Bala \& Purohit S70A (holo BSJO!)

Glumes persistent, upper glume 3-nerved.

Flowering and fruiting: January-February.

Distribution: India: Rajasthan. Endemic.

Habitat: Grows along roadsided, in open land.

Specimen examined: INDIA. Rajasthan: Jaisalmer District: 07.01.2007, Singh, Bala \& Purohit S70A (BSJO).

39. Eragrostis trichodes (Nutt.) Alph. Wood, Classbook Bot., ed. 1861: 796. 1861.

Poa trichodes Nutt., Trans. Amer. Philos. Soc., ser. 2, 5: 146. 1835. Type: USA, s.d., Thomas Nuttall s.n. (holo NY [NY00431374, image!]).

(Fig. 10 \& Plate 4)

Perennial. Culms 30-120 (-160) cm high, erect; nodes brownish; internodes glabrous. Leaf sheaths $c .8 \mathrm{~cm}$ long, villous hairy along margins, mouth bearded. Ligule a fringe of $0.3-0.5 \mathrm{~mm}$ long cilia. Leaf blades $15-46(-65) \times 0.15-0.8 \mathrm{~cm}$, involute, upper side glabrous or sparsely ciliate. Panicles $30-80 \times 6-30 \mathrm{~cm}$, open, oblong to ovoid; primary branches $2-35 \mathrm{~cm}$ long, alternate to subwhorled, capillary; axils ciliate; pedicels up to $1 \mathrm{~cm}$ long, capillary, long, flexuous. Spikelets $3-$ $15 \times 1-3.6 \mathrm{~mm}, 4-18$-flowered, ovate to lanceolate or oblong, greenish to yellowish with pinkish to purplish tinge; rachilla zigzag; florets more or less closely arranged on rachilla, disarticulating from below upwards. Glumes 1.8-4 mm long, subequal, narrowly ovate to linear or lanceolate, membranous, 1-nerved, 1-keeled, apex acuminate. Lemmas 2.2-3.5 mm long, broadly ovate to lanceolate, membranous, 3-nerved, lateral nerves distinct, 1-keeled, keel prominent, 
scabrous along keel towards apex, apex obtuse to acute. Paleas 1.8-2.8 × c. $0.35 \mathrm{~mm}$ long, persistent, 2-nerved, 2-keeled, scaberulous along keels above middle, apex obtuse to truncate; palea flaps almost equal in width to its body. Anthers 3, 1-1.6 mm long, purplish. Caryopses $0.6-1.3 \times$ c. $0.4 \mathrm{~mm}$, oblong, prismatic, ventrally grooved, truncate at ends, dark reddish.

Distribution: INDIA: Introduced (Bor, 1960); USA: ILLINOIS to COLORADO and TEXAS.

Chromosome no.: $2 \mathrm{n}=40$ (Reeder, 1977).

Notes: Bor (1960) mentioned the occurrence of this species in India without its precise locality of distribution in the country. Furthermore, the collection of this species from India could not be traced in any of our herbaria. However, it is included here based on the report of Bor (1960). The description and illustration provided here are based on the USA collections deposited at CAL.

Specimens examined: UNITED STATES OF AMERICA. Kansas, 06.10.1968, Le Roy H. Harvey 8326 (CAL); Kansas, Manhattan, 08.09.1826, F.C. Gates 1095, 1096 (CAL); Oklahoma 05.10.1968, Le Roy H. Harvey 8324 (CAL).

40. Eragrostis unioloides (Retz.) Nees ex Steud., Syn. Pl. Glumac. 1: 264. 1854.

Poa unioloides Retz., Observ. Bot. 5: 19. 1788. Type: INDIA, 1776, Koenig s.n., Herb. Retzius (holo LD, fragment and photo in K \& BRI).

Poa rubens Lam., Tabl. Encycl. 1: 184, t. 45, f. 2.1791. Eragrostis rubens (Lam.) Hochst. ex Miq., Nieuwe Verh. Eerste Kl. Kon. Ned. Inst. Wetensch. Amsterdam ser. 3, 4: 38. 1851. Type: India: Mangalore; Sonnerat s.n. (P-LAM).

Eragrostis amabilis sensu Stapf in Hook.f., Fl. Brit. India 7: 317. 1896, non Wight \& Arn. ex Nees, 1838 (excluding Poa amabilis L., Sp. Pl.: 68. 1753).

Eragrostis unioloides var. tremula Jacob in J. Bombay Nat. Hist. Soc. 47: 51. 1947. Type: INDIA, Kerala, Thiruvalla, 30 m, 27 November 1941, K.C. Jacob 2020 (holo MH!).

(Fig. 11 \& Plate 3)

Annual or perennial. Culms up to $90 \mathrm{~cm}$ high, decumbent, geniculate, rooting at lower nodes; nodes brownish; internodes $c .9 \mathrm{~cm}$ long, green to purplish. Leaf sheaths $c .4 \mathrm{~cm}$ long, margins glabrous or narrowly ciliate. Ligule $c .4 \mathrm{~mm}$ long, a fringe of cilia with narrow membrane at base. Leaf blades $2-5 \times c$. $0.4 \mathrm{~cm}$, linear to lanceolate, upper side scabrid to sparsely tubercle based ciliate. Panicles 5-20 $\times 4-8 \mathrm{~cm}$, open, ovate; primary branches alternate; axils glabrous; pedicels 3-7 mm long. Spikelets $4-16 \times 2-4 \mathrm{~mm}$, up to 72 -flowered, ovate to lanceolate, green to greenish purple or pinkish; rachilla slender, zigzag; florets closely arranged on rachilla, disarticulating from below upwards. Glumes unequal, ovate to lanceolate, 1-nerved, 1-keeled, scabrous along keel, apex acute to subacuminate; lower glume $0.9-1.6 \times 0.2-0.3 \mathrm{~mm}$; upper glume $1.2-1.4 \times$ $0.4-0.5 \mathrm{~mm}$. Lemmas c. $1.3 \times 0.4-1 \mathrm{~mm}$, broadly ovate to elliptic, orbicular when spread, chartaceous, 3nerved, 1-keeled, scabrid along keel, apex acute to mucronate. Paleas $1.2-1.8 \times 0.3-0.8 \mathrm{~mm}$, caducous, ovate to elliptic or oblanceolate, 2-nerved, 2-keeled, narrowly winged and scaberulous along keels; palea flaps narrower than its body. Anthers 2, 0.3-0.4 (-0.5) $\mathrm{mm}$ long, purplish. Caryopses $0.4-0.6 \times 0.2-0.4 \mathrm{~mm}$, obovoid to ellipsoid, reddish brown.

Flowering and fruiting: Almost throughout the year.

Distribution: INDIA: Almost throughout; TROPICAL ASIA, AFRICA, MYANMAR, NEPAL and SRI LANKA.

Habitat: This species occurs chiefly in wetland situations; it also found common along the roadsides, railway tracks, in wastelands, muddy sandy soils, clay and black cotton soils after rainy season, seashore, cultivated fields, fallow fields, rocky areas, etc.

Chromosome nos.: $2 \mathrm{n}=20, \approx 30$ (Larsen, 1963).

Vernacular names: Chota loniya, Loniya, Poi, Poke, Moti chava (Bengali); Chiman chara, Seete-che pohe (Marathi); Banso (Nepali); Udara gaddi (Telugu).

Notes: It is a very variable species in habitat and morphology. Bor (1960) says that some of the Ceylon (Sri Lanka) specimens are perennial though the species is typically annual. Colour of the spikelet varies from purplish to greenish and height of the plant from short to much taller. It has a distribution from sea level up to $1000 \mathrm{~m}$ but with similar inflorescence characters irrespective of differences in elevations.

Specimens examined: INDIA. Andaman and Nicobar Islands: Andamans, 1889, David Prain s.n. (CAL); Little Andaman: Hut Bay, 20.11.1977, N. Bhargava 6503 (CAL); Middle Andaman: Nimbudera, 26.07.1974, N. Bhargava 1872 (CAL); Nicobar: Campbell Bay, 24 Aug.1975, N.P. Balakrishnan 3065 (CAL); Campbell Bay town, 10.10.1980, R.P. Dwivedi 8061 (CAL); Mus, Car Nicobar, 20 Sept.1976, N.C. Nair 4455 (CAL); North Andaman: Diglipur, 05.12.1976, N.P. 
Balakrishnan 4959 (CAL); Mayabundar, 29.01.1959, K. Thothathri 9138 (CAL); South Andaman: Carbyns Cove, 03.10.1973, N.P. Balakrishnan 574 (CAL \& MH). Andhra Pradesh: Ananthapuramu District: Chandragiri Fort, S.V. University, $250 \mathrm{~m}, 04.02 .1989$, Ranga Charyulu 2277 (MH); Garugundukona, $350 \mathrm{~m}$, 21.12.1982, T. Pullaiah 951 (MH); Kalasamudram, 08.02.1983, N. Yesoda 1202 (MH); Khammam District: Bandi Revu Forest, $\pm 200 \mathrm{~m}, 21.09 .1994, R$. Chnadrasekaran 99100 (MH); Kurnool District: Moodavanguluvanka, $300 \mathrm{~m}, 08.12 .1963$, J.L. Ellis 18014 (MH); Srikakulam District: Vandra, 30.11.1961, K.P. Janardhanan 75982 (CAL); Visakhapatnam District: Arakuvalley, $950 \mathrm{~m}, 23.08 .1960$, N.P. Balakrishnan 10782 (CAL \& MH); Khotnighanda area, $910 \mathrm{~m}$, 12.05.1964, G.V. Subbarao 19519 (MH); Warangal District: Pakhal R.F., 290 M, 28.11.1960, K.M. Sebastine 11662 (CAL \& MH). Arunachal Pradesh: Anjaw District: Walong, 08.11.1872, C.B. Clarke 18244 (CAL); Lohit District: 17.08.1969, A.S. Rao 47898 (CAL); Subansiri District: Kherfari, 28.09.1959, G. Panigrahi 19699 (CAL); Thuni Pahad, 24.09.1959, G. Panigrahi 19373 (CAL); Tirap District: Margerita to Jairampur, 10.10.1959, Rolla S. Rao 19923 (CAL); Namchek, 13.10.1959, Rolla S. Rao 20186 (CAL). Assam: Cachar District: Haflong, North Cachar Hills, 24.08.1908, William G. Craib 201 \& 440 (CAL). Bihar: Champaran District: Bhainaloton, 18.09.1965, S.P. Banerjee 658 (CAL); Khangaria District: Mathar, 25.10.1959, B.M. Wadhwa 59794 (CAL). Chhattisgarh: Bastar District: Gidam, 26.09.1962, S.K. Jain 5172 (CAL); Keskal, 16.11.1958, K. Subramanyam 7107 (CAL, MH). Daman: Daman (Grande), 26.09.1963, M.Y. Ansari 3669 (CAL); Daman (peq.), 24.09.1963, M.Y. Ansari 93512 (CAL); Gnaga River bank, 11.05.1963. S.R. Rolla 89233 (CAL). Goa: Sanguem District: Dudhasagar, s.dat., M.Y. Ansari 124039 (CAL); Rivona to Colomba, 20.09.1965, John Cherian 106293 (CAL). Jharkhand: Giridh District: Parasnath, 01.10.1873, C.B. Clarke 21078 (CAL); Sahibganj District: Rajmahal, Oct. 1894, Mokim 1469 (CAL); Ramghur Ghat, 19.10.1883, C.B. Clarke 33887 (CAL); Singhbhum District: s.loc., Dec. 1902, H.H. Haines 520 (CAL). Karnataka: Belgaum District: Molem, 17.09.1970, N.P. Singh 124229 (CAL); Coorg District: Fraserpet, 06.06.1902, C.A. Brber 4405, 4416 \& 4417 (MH); Mysore District: Bandipur Area, 24.08.1964, B.D. Naithani 21120 (CAL \& MH); Biligiri Rangan Hills, 26.10.1978, S.R. Ramesh \& S.B. Manohar 3914 (CAL); Nammar, 29.05.1957, G.S. Puri 19638 (CAL); Vlial Someshwar Beach, 21.11.1972, T.A. Rao 9994 (CAL);
North Kanara District: Siddapur, 05.05.1956, G.S. Puri 1984 \& 1985 (CAL); Uttara Kannada District: Mangalore,.01.1902, C.A. Barber 4808 \& 4848 (MH); Sullia, 25.10.1900, C.A. Barber 2175 (MH). Kerala: Eranakulam District: Willingdon Island, 31.10.1972, T.A. Rao 9656 (CAL); Idukki District: Kulamavu, 23.09.1984, C.N. Mohanan 80144 (MH); Narakkanam, 18.08.1977, K. Vivekanathan 49681 (CAL \& MH); Thekkadi (Periyar Wildlife Sanctuary), 26.11.1987, N. Venhatasubramanyan \& K.R. Sasidharan 13500 (FRC); Kannur District: Ambayathode, 22.01.1979, V.S. Ramachandran 59141 (CAL \& MH); Bekal, 29.01.1979, V.J. Nair 59957 (CAL \& MH); Madayipara, 29.07.2008, K.K. Suresh 04970 (Herbarium of Malabar Botanical Garden and Institute For Plant Sciences, Kozhikode, Kerala); Mattannur, 21.01.1979, V.J. Nair 59771 (CAL, $\mathrm{MH})$; Bela, 01.10.1982, R. Ansari 74416 (CAL); Hosdurg, 07.10.1979, R. Ansari 64785 (CAL \& MH); Kakkadavu, 250 m, 11.05.1982, V.J. Nair 73833 \& 73847A (MH); Kollam District: Anathode, 10.11.1975, K. Vivekananthan 46598 (CAL \& MH); Nilamel, 24.05.1978, C.N. Mohanan 55778 (CAL \& MH); Kottayam District: Changanasseri, Ittithanam 04.02.1984, V.T. Antony 259 (MH); Nagarampara R.F., Ayyappankovil Range, 16.09.1982, K.N. Subramanyan, N. Venkatasubramanyan \& K.R. Sasidharan 8678 (FRC); Ranni-Moozhiyar Konni R.F., 19.10.1983, K.N. Subramanyan 9671 (FRC); Kozhikode District: Aruvampara, 08.10.1979, N.C. Nair 64426 (MH); Palakkad District: Aruvampara slope, 08.10.1979, N.C. Nair 64426 (MH); Kanjarampuzha, 26.10.1964, K.M. Sebastine 22305 (CAL \& MH); Pathanamthitta District: Naduvathumuzhi, 25.08.1913, C.C. Calder \& M.S. Ramaswami 142 (CAL); Thiruvanananthapuram District: Kottur, 175 m, 20.11.1977, M. Mohanan 52589 (CAL \& MH); Thrissur District: Chalakkudi to Thumbermuzhi, 25 m, 15.09.1976, K. Ramamurthy 48527 (CAL \& MH); Travancore: s.d., M.A. Lawson 157 (CAL); Wayanad District: Kammana, 700 m, 03.04.2010, C.P. Vivek 126101; 126102 (MH). Madhya Pradesh: Hosangabad District: Hoshangabad, 28.09.1960, J. Joseph 11062 (MH); Pachmarhi, 28.07.1964, G. Panigrahi \& V.N. Singh 4597 (CAL); Rewa District: Chaihai falls, 16.09.1959, K.M. Sebastine 8834 (CAL \& MH); Khatoulia Forest, Rewa town, 14.09.1959, K.M. Sebastine 9775 (CAL). Maharashtra: Ahmednagar District: Kukadi Junnar River bank, 14.10.1962, M.Y. Ansari 81964 (CAL); Amravati District: Chandrabhaga, Konark Coast, 20.10.1967, A.K. Mukherjee 6036 (CAL); Pune District: Bhimasangar, 12.10.1956, G.S. Puri 8421 (CAL); Bhimasangar Road, 
08.10.1962, K.P. Janardhanan 81667 (CAL); Shinga Hills, Khed Taluka, 27.11.1961, K.P. Janardhanan 75936 (CAL); Wanawadi, 25.09.1965, K. Hemadri 107343 (CAL); Yavatmal District: Kharbi range, Penganga filling series, 23.12.1976, S. Karthikeyan 148549 (BSI). Meghalaya: East Khasi Hill District: Shillong Gauhati Main Road, 16.11.1956, G. Panigrahi 4483 (CAL); Khasi Hills District: Khasia, s.dat., J.D. Hooker s.n., MH Acc. No. 87757 (MH). Nagar Haveli: Minico Islands: Dolara, s.dat., M.Y. Ansari 94111 (CAL). Odisha: Balasore District: Balaramgudi, Odisha Coast, 30.11.1965, T.A. Rao 5642 (CAL); Ganjam District: 20.10.1930, V. Narayanaswami 4706 (MH); Chatrapur, Dec. 1889, J.S. Gamble 21532 (CAL); Kalahadi District: Kanghatta, 22.09.1956, S.K. Mukherjee 4332 (CAL); Kendrapara District: Bhitarkauka, R.F., 06.02.1961, G. Panigrahi 23740 (CAL); Sundargarh District: Bondamunda, 12.09.1988, D. Namhata 3778 (CAL). Rajasthan: Banaswara District: Punapather Forest, 15.10.1976, V. Singh 3657 (CAL). Sikkim: East District: Chalisay, on way to Rongli, 07.12.1980, P. Chakraborthy 1021 (BSHC); Gangtok, 06.09.1968, N.C. Majumdar 258 (CAL); East Himalaya: Muigfoo, 01.10.1940, K. Biswas 4476 (CAL); Simulbari, 16.11.1948, K. Biswas 8733 (CAL); North Sikkim: Thingvul, 10.10.1985, Desh Ray 4803 (BSHC). Tamil nadu: Coimbatore District: Aliyar submergible area, 350 m, 21.11.1962, K.M. Sebastine 15095 (MH); Bolampatti, 28.09.1910, C.E.C. Fischer 2250 (FRC); Kallar, 10.07.2010, C.P. Vivek \& S. Arumugam 126130 (MH); Poonachi Ghat, Anamalais, 08.10.1901, C.A. Barber 3560 (MH); Walayar Dam Area, 04.09.2010, C.P. Vivek 126142 (MH); Cuddalore District: Kovalanthittu, Pichavaram R.F., 04.03.1970, K.N. Subramanyan 3920 (FRC); Dindigul District: Kodaikkanal, 11.10.1919, K.C. Jacob 16019 (MH); Erode District: Kurubithi Halla forest, 26.06.1988, N. Venkatasubramanyan 1282 (FRC); Krishnagiri District: Hosur Cattle Farm, 09.06.1930, V. Narayanaswami 3054 (MH); Madurai District: Tiruparangundram, 225 m, 06.05.1984, K. Ravikumar 1757 (MH); Nilgiris District: Avalanche, 14.06.1970, B.V. Shetty $34230(\mathrm{MH})$; Bikkapathimund shola, 26.03.1972, G.V. Subbarao 40492 (MH); Coonoor, Feb. 11, Prain s.n. (CAL); Coonoor, Sims Park, 28.07.1957, K.M. Sebastine 4079 (CAL \& MH); Ootacamund, 27.08.1901, C.A. Barber 3481 (MH); Pudukkottai District: Sithannavasal, 120 m, 28.11.1984, C. Arulappan 260 (MH); Thanjavur District: Kodikkavai, sea level, 27.02.1987, S. Ragupathy 227 (MH); Tirunelveli District: Ambasamudram, 13.05.1901, C.A.
Barber 2744 (MH); Singapatty, Virakulam, $66 \mathrm{~m}$, 02.03.1958, K.M. Sebastine 5483 (MH); Sivanaperi, 225 m, 12.11.1962, J. Joseph 15271 (CAL, MH); Tiruchirappalli District: Pachimalais, Thuraiyur Taluka, 16.03.1976, K.M. Matthew 12307 (CAL); Puliamcholai, 333 m, 06.08.1958, K.M. Sebastine 6192 (CAL, MH). Tripura: Agartala: Agartala Road, 25.08.1957, R.S. Rao 8813 (CAL); Charilam, Agartala, 04.03.1960, D.B. Deb 2518 (CAL). Uttarakhand: Kumaon District: Dafia Dhoon, 2500 m, C.M. Arora 53272 (BSD); Garhwal District: Batwalchari, $1000 \mathrm{~m}$, 20.09.1958, M.A. Rao 6397 (BSD); Trinula, 1200 m, 24.10.1970, B.D. Naithani 42294 (BSD); Dehra Dun District: Robber's cave, 29.07.1964, C.R. Babu 33258 (BSD); Hathibarkala, 700 m, 19.09.1956, T.A. Rao 879 (BSD). Uttar Pradesh: Gorakhpur District: Barwa, 02.11.1963, C.M. Arora 1476 (CAL); Kumaun Division, Askot, 1550 m, 01.09.1971, C.M. Arora 45474 (BSD); Dafia Dhoon, 2500 m, 03.09.1973, C.M. Arora 53272 (BSD); Lauwand, Dhudi, 24.07.1965, O.P. Misra 9937 (CAL); Lakshmipur Kheri District: Mailani, 17.12.1960, C.L. Malhotra 13370 (BSD); Pilibhit District: Amaria, 02.10.1970, C.L. Malhotra 42410 (BSD); Bisalpur, 21.10.1970, C.L. Malhotra 42893 (BSD); Madhotanda, 20.10.1970, C.L. Malhotra 42862 (BSD); Shahi, 03.10.1970, C.L. Malhotra 42506 (BSD); Saharanpur District:.09.85, T.S. Murthy \& A.K. Goel 1152 (BSD); Saharanpur, 13.10.1887, J.F. Duthie s.n., CAL Acc. No. 541567 (CAL); Nisharigara, 24.11.1964, G. Panigrahi \& O.P. Misra 6599 (CAL). West Bengal: Birbhum District: Nalhati, 15.12.1966, R.K. Basak 633 (CAL); Santiniketan, 16.10.1968, R.K. Basak 633 (CAL); Darjeeling District: Jaldhaka, 22.02.1910, Ribu \& Rhomoo 3657 (CAL); Dinajpur District: Baro Raghunathpur, Ralurghat, 25.10.1983, R.N. Banerjee 15958 (CAL); Chopra West, 26.08.1984, R.N. Banerjee 18728 (CAL); Malda District: Kisli, 14.03.1962, J.K. Maheshwari 4911 (CAL).

41. Eragrostis viscosa (Retz.) Trin., Mem. Acad. Imp. Sci. St. Petersbourg., Ser. 6, 1(4): 397. 1830.

Poa viscosa Retz., Observ. Bot. 4 : 20. 1786 -1787. Eragrostis tenella var. viscosa (Retz.) Stapf in Hook.f., Fl. Brit. India 7: 315. 1896. Type: INDIA, Malabar, Koenig s.n. in Herb. Retzius (holo LD, fragment K [K000643369, image!], iso BM [BM000578756, image!]).

(Fig. 11 \& Plate 4)

Annual or perennial. Culms $22-76 \mathrm{~cm}$ high, erect to decumbent, geniculate, viscous; nodes brownish; internodes 3-13 cm long with glandular patches all along or glandular ring or spots close below nodes, 
more or less viscous. Leaf sheaths $c .3 \mathrm{~cm}$ long, viscous around mouth, more or less ciliate along one margin, mouth bearded. Ligule a fringe of cilia. Leaf blades 8$20 \times 0.2-0.5 \mathrm{~cm}$, linear to lanceolate, upper side scabrid, lower side with or without tubercle based cilia. Panicles $7-20 \times 1.5-6 \mathrm{~cm}$, open to narrowly spiciform, ovate; primary branches alternate with glandular patches, more or less viscous; axils glabrous, at times ciliate; pedicels $0.5-2 \mathrm{~mm}$ long, glandular. Spikelets $2-5 \times$ 0.9-1.5 mm, 5-11(-12)-flowered, ovate to lanceolate or oblong, light green or green with purple shade; rachilla slender, zigzag; florets more or less closely arranged on rachilla, disarticulating from above downwards. Glumes ovate to lanceolate, more or less viscous, margins at times minutely serrulate, chartaceous, 1-nerved, 1-keeled, scabrid along keel, apex acute; lower glume $0.5-1 \times 0.2-0.4 \mathrm{~mm}$; upper glume $0.9-1 \times$ c. $0.4 \mathrm{~mm}$. Lemmas $0.9-1.1 \times 0.4-0.6$ $\mathrm{mm}$, ovate to elliptic, chartaceous, 3-nerved, 1-keeled, scabrid along keel towards apex, apex acute. Paleas 0.9$1 \times 0.3-0.4 \mathrm{~mm}$, caducous, elliptic, 2-nerved, 2-keeled, ciliate along keels, apex acute to obtuse; cilia on palea keels ascending, equal to or shorter than width of palea body; palea flaps narrower than its body. Anthers 3, 0.15-0.4 mm long, purplish. Caryopses 0.4-0.5 × 0.2$0.3 \mathrm{~mm}$, ovoid to ellipsoid, reddish brown.

Flowering and fruiting: Throughout the year.

Distribution: INDIA: Almost throughout; TROPICAL and SOUTH AFRICA, PHILIPPINES, MALAYSIA, MYANMAR and SRI LANKA.

Habitat: On roadsides, in wastelands, drier areas, in open grasslands and sandy seashores.

Etymology: The specific epithet 'viscosa' means viscid, because of the glands all over the plant body.

Vernacular names: Bhurbur, Bhurbusi, Chikti, Chipal (Bengali), Bankasigarantha (Telugu).

Notes: This is a highly variable species with three forms in India. However, panicles of each form are apparently different. The most common viscous form is distributed in drier areas which has spreading panicle. The second form is commonly distributed in wetlands and costal areas that have linear and narrow panicle. This form is comparatively less viscous and resembles E. tenella., but different from the latter by the comparatively larger spikelets. Third form has spiciform and viscous panicle as in E. riparia while the latter species has more condensed and non-viscous panicle.

The combination made by Trinius in 1830 for this species is valid. Thus the same combination done by Nees ex Wight \& Arnott in Wight's Catalogue: 106 (1834) is superfluous (Noltie, 2005).

Specimens examined: INDIA. Andhra Pradesh: Ananthapuramu District: S.K. University, $350 \mathrm{~m}$, 21.10.1982, N. Yesoda 875 (MH); Seshachalam, 26.11.1917, s.coll. $15364(\mathrm{MH})$; Chittoor District: Varadaya Palem to Kambakam, 125 m, 27.09.1974, M. Chandrabose 45157 (MH); East Godavari District: Annavaram, 20.01.1916, s.coll. 12620 (MH); Annavaram, 20.01.1916, C.E.C. Fischer s.n. (FRC); Mundapetta, 17.03.1902, C.A. Barber 4290 (MH); Karimnagar District: Aklaspur, 200 m, 30.12.1964, G.V. Subbarao 22491 (MH); Krishna District: Mylavaram, 04.08.1907, s.coll. s.n. (MH); Kurnool District: Gazulapalle, 30.08.1936, K.C. Jacob s.n. (MH); Nellore District: 21.12.1917, C.E.C. Fischer 4243 (CAL); March 1918, C.E.C. Fischer 4307 (CAL); Bhintaldevi, 06.01.1920, s.coll. 16398 (MH); Duttalur, March 1918, C.E.C. Fischer 4305 \& 4307 (FRC); West Godavari District: Pedda-Ellore, 09.10.1930, V. Narayanaswami 4513; 4517 (MH). Bihar: Shahabad District: Nov. 1875, C.B. Clarke 27415 (CAL). Karnataka: Belgaum District: Dharwar, 22.11.1957, G.H. Khisti 29640 (CAL); Mysore District: Bangalore, Oct. 1916, R.D. Anstear s.n. (MH); North Canara District: 29.11.89, W.A. Talbot 2102 (CAL); South Canara District: Udupi (Udyavar), 12.02.1952, A. Sunanda kamath s.n. (MH). Kerala: Kannur District: Muzhuppilangad, 50 m, 16.12.1979, V.S. Ramachandran 65262 (CAL \& MH); Nileshwar, 29.01.1979, V.J. Nair 59985 (CAL); Tellichery-near paddy field, $\pm 50 \mathrm{~m}, 15.07 .1978$, V.S. Ramachandran 57665 (MH); Tellichery, $50 \mathrm{~m}, 16.08 .1980$, V.S. Ramachandran 66982 (MH); Kollam District: Kodumon, 125 m, 02.09.1978, C.N. Mohanan 54959 (MH); Paravur, sealevel, 23.05.1978, C.N. Mohanan 55754 (MH); Thenmalai, $450 \mathrm{~m}, 19.05 .1978$, C.N. Mohanan 55594 (MH); Travancore: Chivarambu, 28.11.1941, K.C. Jacob 20211 (MH); Kozhikode District: Malabar Botanical Garden, 09.09.1999, P.M. Krishnan 784 (Herbarium of Malabar Botanical Garden \& Institute for Plant Sciences, Kozhikode, Kerala); Malaparamba, 30.05.2010, C.P. Vivek 126116 (MH). Maharashtra: Gharodi, J.H. Burkill 16326 (CAL); Mukuthan: 14.12.1976, S. Karthikeyan 148425 (BSI). Odisha: Lampda, 22.12.1960, P.C. Nanda 2443 (CAL). Rajasthan: Sirohi District: Gurusikhar-On the way, 10.11.1959, S.K. Jain 60185 (BSI). Tamil Nadu: Chengalpattu District: Sithamur, 28.10.1914, s.coll. 11198 (MH); Coimbatore District: Aliar Dam nearby, 650 m, 11.05.2011, C.P. Vivek $126150(\mathrm{MH})$; Bharathiar 

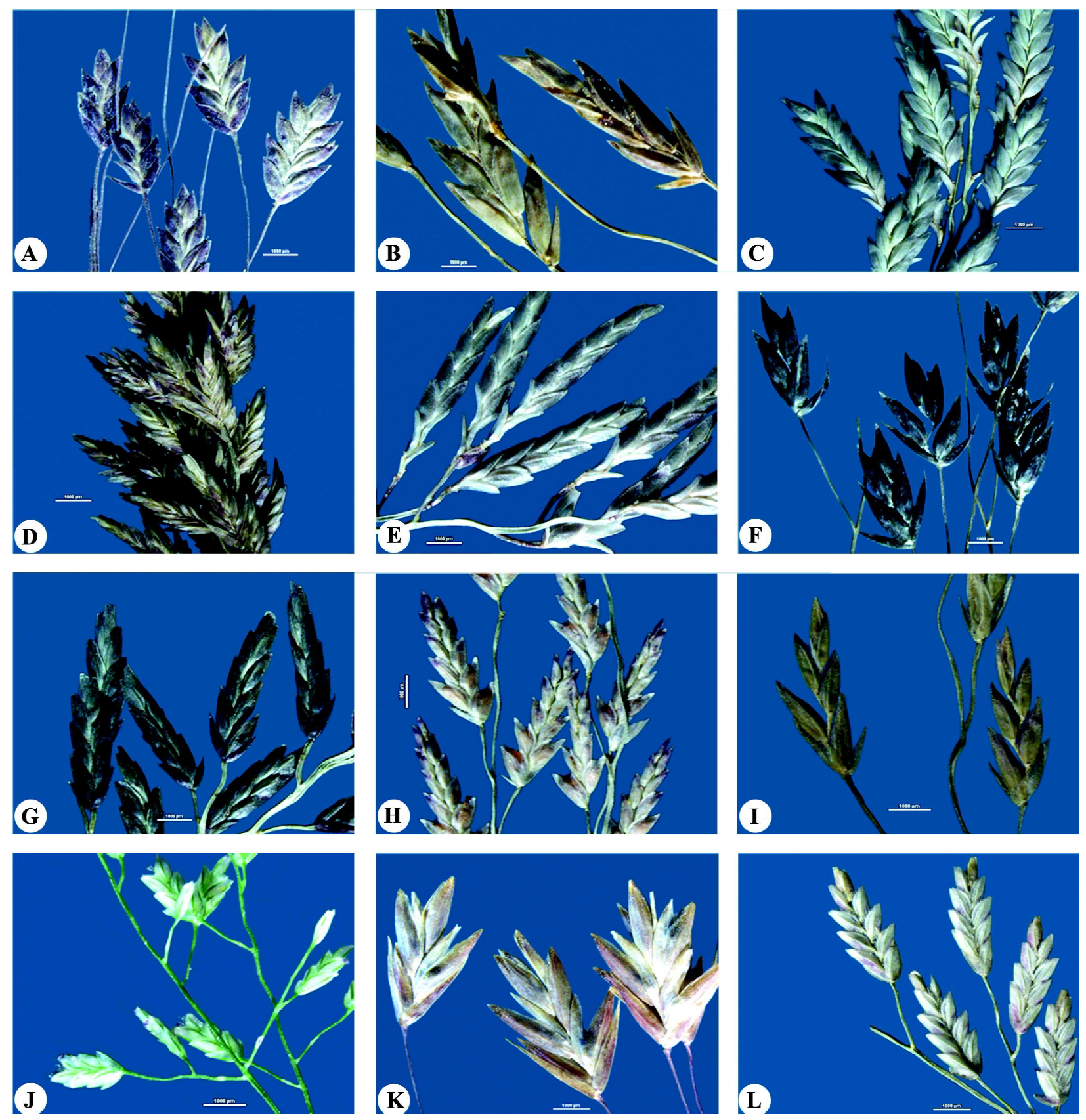

Plate 4. Spikelets of : A. Eragrostis burmanica Bor; B. E. ferruginea (Thunb.) P. Beauv.; C. E. gangetica (Roxb.) Steud.; D. E. japonica (Thunb.) Trin.; E. E. maderaspatana Bor; F. E. nigra Nees ex Steud.; G. E. nilgiriensis Vivek, G.V.S. Murthy \& V.J. Nair; H. E. pilosa (L.) P. Beauv.; I. E. tef (Zucc.) Trotter; J. E. tenella (L.) P. Beauv. var. tenella; K. E. trichodes (Nutt.) Wood; L. E. viscosa (Retz.) Trin. 
University Campus, 27.06.2010, C.P. Vivek 126121 (MH); 22.07.2010, C.P. Vivek $126138(\mathrm{MH})$; Burliar, 11.05.2010, C.P. Vivek, 126111 (MH); Maruthamalai, 653 m, 14.11.1956, K.M. Sebastine 1287 (CAL \& MH); Thekkumalai, 09.07.1956, K.M. Sebastine 200 (CAL); Velliangiri Temple nearby, $600 \mathrm{~m}$, 23.10.2011, C.P. Vivek 126158 (MH); Cuddalore District: Without precise locality, 12.03.1903, C.A. Barber 6062 (MH); 19.02.1979, K. Ramamurthy 60318 (CAL); Kanchipuram District: Echankadu-Pelabuppam, sealevel, 21.08.1930, V. Narayanaswami 4083 (MH); Palur, 23.08.1930, V. Narayanaswami 4083 (MH); Oragadam, 19.12.1916, s.coll. $14080(\mathrm{MH})$; Madurai District: 27.10.1956, S.C. Agarwal 625 (CAL); Nupuraganga-On the way, $167 \mathrm{~m}, 11.06 .1957$, s.coll. 3385 (MH); Ramanathapuram District: Alagar Koil Forest, 300 m, 27.07.1965, E. Vajravelu 25287 (MH); Chitrangudi, \pm 75 m, 24.05.1988, V. Balasubramanyam 1778 (CAL \& $\mathrm{MH}$ ); KrishnathevarthoppuMamsapuram, 225 m, 09.03.1980, S.R. Srinivasan 63635 (MH); Krusadai, 30.01.1945, D. Daniel \& S.R. Raju s.n. (MH); Perunazhi, $75 \mathrm{~m}, 22.02 .1988, V$. Balasubramanyam 1632 (MH); Peruvayal, $10 \mathrm{~m}$, 16.01.1989, V. Balasubramanyam 2118 (MH); Sayalgudi, 90 m, 07.12.1986, V. Balasubramanyam 53179 (MH); Thiruppullani, 16.05.1944, S.N. Chandrasekaran \& S.V. Parthasarathi 87123 (MH); Thiruvannamalai District: Chettupatu to Pennathur, 75 m, 19.02.1979, K. Ramamurthy 60318 (MH); Thoothukkudi District: Nazareth, 11.07.1899, C.A. Braber 662 (MH); Villakku Road, 50 m, 07.02.87, $V$. Balasubramanyam 1186 (MH); Tippukadu R.F., 165 m, 25.11.1963, 17691 (MH); Tirunelveli District: Alankolam, 30.06.1901, C.A. Barber 3345 (MH); Ambasamudram, 13.05.1901, C.A. Braber 2748 (MH); Manimuthar Dam area, 300 m, 24.06.1957, K.M. Sebastine $3562(\mathrm{MH})$; Mundanthorai Ghat, 14.05.1901, C.A. Braber 2752 (MH); Muthaliavattu-Srivilliputhur, 19.09.1917, s.coll. 15014 (MH); Palamkotta, 11.05.1901, C.A. Barber 2716 (MH); Vadugapatti, 29 Apr. 2010, C.P. Vivek 126104 \& 126105 (MH). Uttarakhand: Dehra Dun District: Dak Pathar, 15.07.1986, J.P. Sharma 70379 (BSD); Rispana, 08.11.1964, C.R. Babu 34632 (BSD). Uttarpradesh: Aligarh District: Gangetic Plain, 07.11.1887, J.F. Duthie 6780; 6785 (CAL); Saharanpur District: 11.10.1887, J.F. Duthie 6781 (CAL); Elmah, 26.11.1886, J.F. Duthie 6598 (CAL).

42. Eragrostis zeylanica Nees \& Mey., Nov. Actorum Acad. Caes. Leop.-Carol. Nat. Cur. 19(Suppl. 1): 204. 1843. Type: SRI LANKA, July 1829, Macrae s.n. (holo herb. Lindley and herb. Arnott, picture K; iso BM [BM000959607, image!]).

Eragrostis elongata sensu Stapf in Hook.f., Fl. Brit. India. 7: 319. 1896, non Jacq., 1813. (Fig. 11 \& Plate 3)

Perennial. Culms 5-25 cm high, erect to narrowly decumbent, geniculate; nodes brownish; internodes $c$. $4 \mathrm{~cm}$ long. Leaf sheaths c. $2 \mathrm{~cm}$ long, slightly ciliate along one margin. Ligule a fringe of cilia with a narrow membrane at base. Leaf blades $2.4-2.6 \times 0.1-0.2 \mathrm{~cm}$, linear to lanceolate, upper surface scabrid. Panicles 3$15 \times 2-4 \mathrm{~cm}$, open, with spikelets fascicled and grouped in branches; primary branches $0.5-1 \mathrm{~mm}$ long, fascicled, start dividing from near base; axils slightly ciliate; pedicels up to $5 \mathrm{~mm}$ long. Spikelets $5-20 \times 0.15-$ $3 \mathrm{~mm}, 10-60$-flowered, subsessile, lanceolate to oblong, apex sharply acute; rachilla narrowly zigzag to almost straight; florets firmly arranged on rachilla, disarticulating from below upwards. Glumes linear to lanceolate, chartaceous or coriaceous, 1-nerved, 1keeled, scabrid along keel towards apex, apex acuminate; lower glume $1-1.5 \times 0.3-0.5 \mathrm{~mm}$; upper glume $1.5-2.5 \times$ c. $0.5 \mathrm{~mm}$. Lemmas $1.5-2.5 \times 1-1.5$ $\mathrm{mm}$, ovate to lanceolate, coriaceous, 3-nerved, nerves prominent and straight, 1-keeled, scabrid along keel towards apex, apex subacute to acuminate. Paleas 1$1.5 \times 0.3-1 \mathrm{~mm}$, persistent, elliptic, slightly curved, 2nerved, 2-keeled, ciliolate along keels above middle; palea flaps narrower than its body. Anthers 3, 0.25-0.3 $\mathrm{mm}$ long, yellowish. Caryopses $0.4-0.5 \times 0.3-0.45 \mathrm{~mm}$, ovoid to subglobose or orbicular, terete to laterally compressed, light brown.

Flowering and fruiting: August-October.

Distribution: INDIA: Assam, Kerala, Madhya Pradesh, Sikkim, Uttar Pradesh and West Bengal; BANGALADESH, MYANMAR and SRI LANKA.

Habitat: On roadsides, banks of streams and backwaters, also in open grasslands.

Chromosome nos.: $2 \mathrm{n}=36,40,60,80$ (Larsen, 1963).

Notes: Eragrostis zeylanica closely resembles E. brownii and $E$. viscosa but clearly differs from both by its short spreading habit, fascicled spikelets, subsessile pedicels and more number of florets in each spikelets.

Specimens examined: INDIA. Kerala: Kannur District: Hosdurg, 125 m, 29.01.1979, V.S. Ramachandran 60035 (CAL \& MH); Palakkad District: Malampuzha Dam site, 23.09.2012, C.P. Vivek 126160 (MH). Sikkim: East Sikkim District: Bendang, 01.11.1988, R.C. Srivastava 


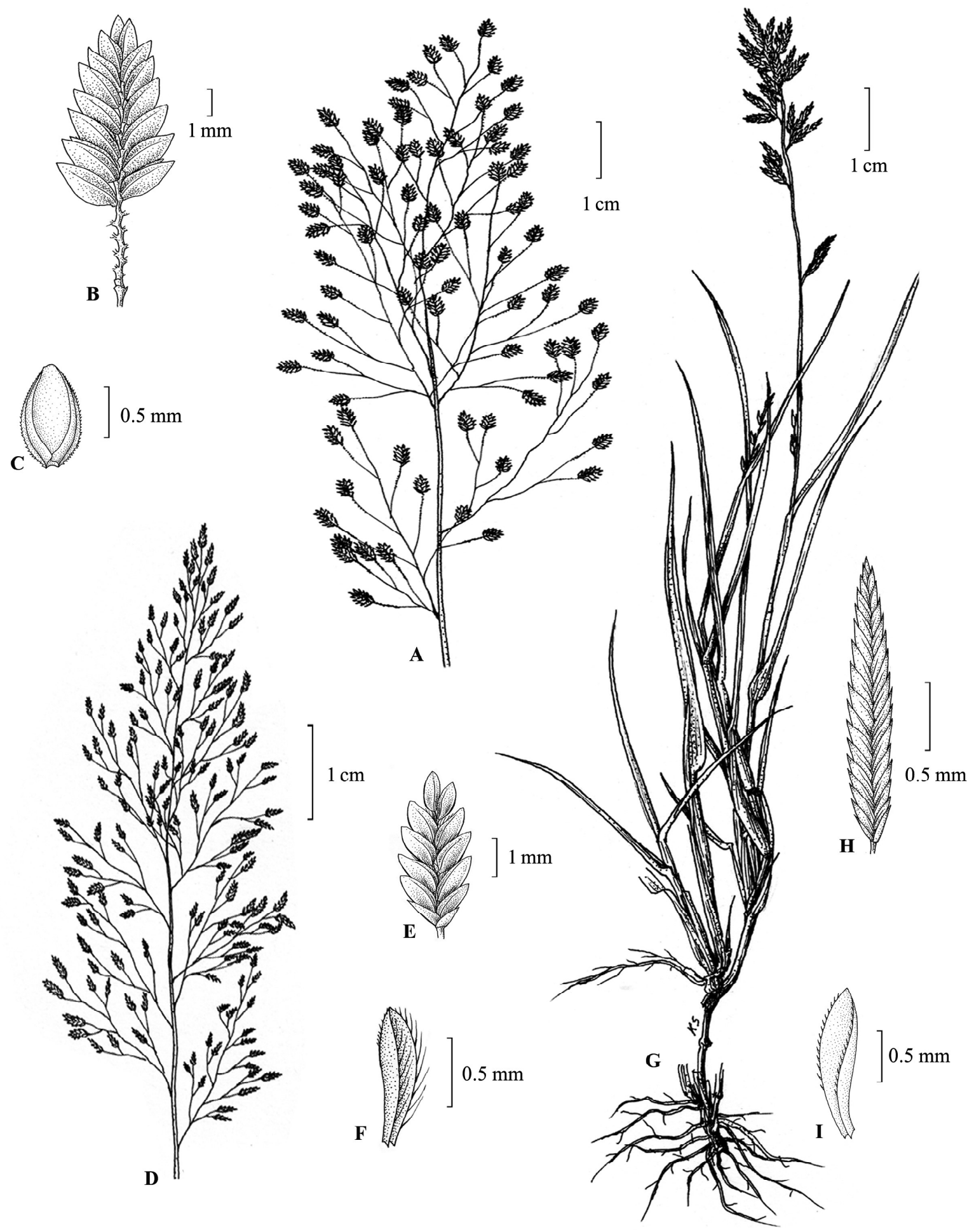

Fig. 11. Eragrostis unioloides (Retz.) Nees ex Steud. (A-C). A. Inflorescence; B. Spikelet; C. Palea-ventral view. E. viscosa (Retz.) Trin. (D-F). D. Inflorescence; E. Spikelet; F. Palea-lateral view. E. zeylanica Nees \& Mey. (G-I). G. Habit; H. Spikelet; I. Palea-lateral view. 
12114, 12118 (BSHC). West Bengal: without precise locality, 1836-38, Joh. W. Helfer s.n. (CAL).

\section{ACKNOWLEDGEMENTS}

The authors wish to thank the Director, Botanical Survey of India for facilities and encouragement. They are thankful to the Ministry of Environment, Forest and Climate Change, Govt. of India, New Delhi for the financial support provided under their All India Coordinated Project in Taxonomy (AICOPTAX). They are also thankful to the Curators of B, BM, BRI, E, FI, K, LD, L, LE, LINN, NTM, NY, P, UPS and W, to locate the specimens; Dr. Kanchi N. Gandhi, Senior Nomenclatural Registrar, Harvard University Herbaria, USA, for nomenclatural clarifications on Eragrostis tenella and E. tenuifolia; Mr. K. Sivanandan, Retired Artist, BSI, SRC, for illustrations.

\section{REFERENCES}

BAQUAR, S.R. AND M. SAEED. 1969. Chromosome studies and polyploidy analysis in grasses of West Pakistan I. Caryologia 22: 103-111.

BEETLE, A.A., E.M. FORCECK, J.A.M. SANCHEZ, V. LUQUE, A.C. HERNANDEZ AND A.M. RODRIGUEZ. 1991. Eragrostis Wolf. In: Las Gramineas de Mexico,Tomo III. COTECOCA, S.A.R.H., Mexico: 50-97.

BOECHAT. S. De C. AND H.M. LONGHI-WAGNER. 2000. Padroes de distribuica geografica dos taxon eiros de Eragrostis (Poaceae, Chloridoideae). Rev. Brazil. Bot. 23: 177-194.

BOECHAT, S. De C. AND H.M. LONGHI-WAGNER. 2001. Ogenero Eragrostis (Poaceae) no Brasil. Iher. ser. Bot. 55:23-169.

BOR, N.L. 1960. The Grasses of Burma, Ceylon, India and Pakistan (excluding Bambuseae). Pergamon Press, London.

BORRE, A. VAN DEN AND L. WATSON. 1994. The infrageneric classification of Eragrostis (Poaceae). Taxon 43: 383-422.

BOWDEN, W.M. AND H.A. SENN. 1962. Chromosome numbers in 28 grass genera from South America.
Canad. J. Bot. 40: 1115-1124.

CHAISONGKRAMA, W., P. CHANTARANOTHAIA AND T.R. HODKINSONB. 2013. A taxonomic revision of the genus Eragrostis in Thailand. Sci. Asia 39: 111-123.

CHEN, C.C. AND C.C. HSU. 1962. Cytological studies on Taiwan grasses. 2. Chromosome numbers of some miscellaneous tribes. J. Jap. Bot. 37: 300-313.

CHRISTOPHER, J. 1976. In: IOPB chromosome number reports LII. Taxon 25: 341-346.

CHRISTOPHER, J. AND A. ABRAHAM. 1974. Studies on the cytology and phylogeny of South Indian grasses. 11. Subfamily Eragrostoideae. Cytologia 39: 561-571.

CLAYTON, W.D AND S.A. RENVOIZE. 1986. Genera Graminum. Grasses of the World. Kew Bull. Addit. Ser. 13: 1-389.

COOKE, T. 1908. The Flora of the Presidency of Bombay. Vol. 2. Francis \& Taylor, London.

COPE, T.A. 1982. Poaceae. In: NASIR, E. AND S.I. ALI (eds.), Flora of Pakistan 143: 1-678. University of Karachi, Karachi.

COPE, T.A. 1998. A synopsis of Eragrostis Wolf (Poaceae) in the flora Zambesiaca area. Kew Bull. 53: 129-164.

COVAS, G. 1991. Taxonomía y morfología del pasto llorón [Eragrostis curvula (Schrad.) Nees], con referencias sobre otras especies cultivadas de Eragrostis. In: FERNÁNDEZ, O., R. BREVEDAN AND A. GARGANO (eds.), El pasto llorón, su biología y manejo. CERZOS and Universidad Nacional del Sur, Bahía Blanca, Argentina, 7-17. da Silva Meyer, A., A. Franco García.

DAVIDSE, G. 1994. Eragrostis Wolf. In: DAVIDSE, G., M., SOUSA AND A.O. CHATER, (eds.), Flora Mesoamericana 6: 263-272. Missouri Botanical Garden Press, St. Louis.

DESHPANDE, U.R. AND N.P. SINGH. 1986. Grasses of Maharashtra. Mittal Publications, New Delhi.

De WET, J.M.J. 1954. Chromosome numbers of a few South African grasses. Cytologia 19: 97-103. 
De WET, J.M.J. 1960. Chromosome numbers and some morphological attributes of various South African grasses. Amer. J. Bot. 47: 44-50.

DUJARDIN, M. 1978. Chromosome numbers of some tropical African grasses from Western Zaire. Canad. J. Bot. 56: 2138-2152.

DUJARDIN, M. 1979. Additional chromosome numbers and meiotic behavior in tropical African grasses from Western Zaire. Canad. J. Bot. 57: 864-876.

ESPEJO-SERNA, A., A.R. LOPEZ-FERRARI AND J.VALDES-REYNA. 2000. Poaceae. In: A. ESPEJO SERNA AND A.R. LOPEZ-FERRARI, eds. Las Monocotyledoneas Mexicanus: una synopsis floristica, Partes IX-XI. Consejo Nacional de la Flora de Mexico, A.C., Univerisidad Autonoma Metropolitanalzapalapa, and Comision Nacional para el conocimiento y uso de la Biodiversidad, Mexico, D.F. $10: 8-236$.

FISCHER, C.E.C. 1934-1936. Gramineae. In: Flora of the Presidency of Madras. Part X and XI. Adlard \& Son Ltd., London.

GIRALDO-CANAS, D., P.M. PETERSON AND I. SANCHEZ VEGA. 2012. The genus Eragrostis (Poaceae: Chloridoideae) in northwestern South America (Colombia, Ecuador, and Peru): Morphological and taxonomic studies. Bibliot. José Jerónimo Triana 23: 1-180.

GRATEROL, A., P. TORRECILLA AND B. TRUJILLO. 1989. Critical comments on the genus Eragrostis Wolf in Venezuela and a key to the identification of its species. Ernstia 51: 16-28.

GOULD, F.W. AND T.R. SODERSTROM. 1974. Chromosome numbers of some Ceylon grasses. Canad. J. Bot. 52: 1075-1090.

HAINES, H.H. 1921-1925. The Botany of Bihar and Orissa. Adlard \& Son \& West Newman Ltd., London.

HARTLEY, W. AND C. SLATER. 1960. Studies on the origin, evolution and distribution of the Gramineae III. The tribes of the subfamily Eragrostoideae. Austral. J. Bot. 8: 256-276.
HALVORSEN, W.L. AND P. GUERTIN. 2003. Fact sheet for: Eragrostis curvula (Schrad.) Nees and Eragrostis curvula var. conferta Stapf. USGS Weeds in the West project: Status of Introduced Plants in Southern Arizona Parks Sonoran Desert Field Station, University of Arizona, Tuson, USA. http://sdrsnet.srnr. arizona.edu/ data/sdrs/ww/docs/eraglehm.pdf.

HITCHCOCK, A.S. 1908. Types of American grasses: a study of the American species of grasses described by Linnaeus, Gronovius, Sloane, Swartz \& Michaux. Contr. US Natl. Herb. 12: 121.

HOST, N.T. 1809. Icones et descriptiones graminum austriacorum... 4:14-15, t. 24. Schmidt, Vienna.

HOUINATO, M., C. DELVAUX AND L. PAUWELS. 2000. Les Eragrostis (Poaceae) du Bénin. Belg. J. Bot. 133: $21 " 35$.

INGRAM, A.L. 2010. Evolution of leaf blade anatomy in Eragrostis (Poaceae). Syst. Bot. 35 (4): 755-765.

INGRAM, A.L. AND J.J. DOYLE. 2007. Eragrostis (Poaceae): monophyly and infrageneric classification. Aliso 23: 595-604.

JAIN, S.K. 1968. Notes on Indian grasses X. Proliferation in Eragrostis Beauv. and Bromus L. Bull. Bot. Surv. India 10:229-230.

JONES, B., J. PONTI, A. TAVASSOLI AND P. DIXON. 1978. Relationships of the Ethiopian cereal Tef (Eragrostis tef (Zucc.) Trotter): evidence from morphology and chromosome number. Ann. Bot. 42: 1369-1373.

KABEER, K.A.A. AND V.J. NAIR. 2009. Flora of Tamil $\mathrm{Nadu}$ - Grasses. Botanical Survey of India, Kolkata.

KALIDASS, C., R. KOTTAIMUTHU, R. AND P.C. PANDA. 2019. Updated checklist of the genus Eragrostis Wolf (Poaceae) in Eastern Ghats, India. Pl. Sci. Res. 41(1\&2): 44-47.

KAMI, E. 1993. The genus Eragrostis in Congo (Central Africa). Belj. J. Bot. 126:33-44.

KAMMACHER, P., G. ANOMA, E. ADJANOHOUN AND L. AKE ASSI. 1973. Nombers chromosomiques 
de graminees de Cote - d' Ivoire. Candollea 28: 191-217.

KARTHIKEYAN, S., S.K. JAIN, M.P. NAYAR AND M. SANJAPPA. 1989. Poaceae. In: Florae Indicae Enumeratio Monocotyledonae. Botanical Survey of India, Calcutta.

LAKSHMINARASIMHAN, P. 1996. Flora of Maharashtra State. Monocotyledons. (edited by KARTHIKEYAN, S. AND N.P. SINGH). Botanical Survey of India, Calcutta.

LARSEN, K. 1963. Studies in the flora of Thailand. 14. Cytological studies in vascular plants of Thailand. Dansk Bot. Ark. 20: 211-275.

LAZARIDES, M. 1972. A revision of Australian Chlorideae (Gramineae). Austral. J. Bot. Suppl. Ser. 5: 1-51.

LAZARIDES, M. 1994. Eragrostis. In: M.S. DASSANAYAKE (ed.), Revised handbook of the flora of Ceylon 8: 212. Amerind, New Delhi.

LAZARIDES, M. 1997. A revision of Eragrostis (Eragrostideae, Eleusininae, Poaceae) in Australia. Austral. Syst. Bot. 10: 77-187.

MALIK, C.P. AND R.C. TRIPATHI. 1970. In: IOPB Chromosome number reports XXVII. Taxon 19: 437442.

MEHRA, P.N., P.K. KHOSLA, B.L. KOHLI AND J.S. KOONAR. 1968. Cytological studies in North Indian grasses. I. Res. Bull. Punjab Univ.19: 157-230.

MOFFETT, A.A. AND R. HURCOMBE. 1949. Chromosome numbers of South African grasses. Heredity 3: 369-373.

MOULIK, S. 1997. The Grasses and Bamboos of India. Vol. 1 \& 2. Scientific Publishers, Jodhpur.

MUKHERJEE, P. 1977. Studies on the karyotype of Eragrostis pilosa Beauv. Proc. 64th Indian Sci. Congr. Part 3: 152.

MUKHERJEE, P. 1978. Karyotype of Eragrostis pilosa. Bull. Bot. Soc. Bengal 32: 63-65.

MULAY, B.N. AND D. JAGADISAN. 1956. Morphology and number of chromosomes in some desert grasses. Proc. 43rd Indian Sci. Congr. 3: 259.
MULAY, B.N. AND M.K. PRASAD. 1956. Chromosome number of some desert grasses. Proc. 43 rd Indian Sc. Congr. 3: 258-259.

MULAY, B.N. AND P.J. LEELAMMA. 1956. Chromosome number of some desert grasses. Proc. Rajasthan Acad. Sci. 6: 65-69.

MUNRO, W. 1862. Eragrostis amabilis. J. Linn. Soc., Bot. 6: 43.

NEES, C.G.D. VON 1829. Agrostologia Brasiliensis. In: MARTIUS, C.F.P. DE (ed.), Flora brasiliensis seu enumeration plantarum 2: 512. Cotta, Stuttgart, Tubingen.

NICOLSON, D.H., C.R. SURESH AND K.S. MANILAL. 1988. An interpretation of Rheede's Hortus Malabaricus. Regnum Veg. 119.

NICORA, E.G. 1998. Revision of the genus Eragrostis Wolf (Gramineae-Eragrostideae) from Argentina and neighbouring countries. Boissiera 54: 5-109.

NOLTIE, H.J. 2005. The Botany of Robert Wight. A.R.G. Gantner Verlag, Liechtenstein.

ONO, H. AND T. TATEOKA. 1953. Karyotaxonomy in Poaceae. I. Chromosome and taxonomic relations in some Japanese grasses. Bot. Mag. 66: 18-27.

PETERSON, P.M. 2003. Eragrostis. In: M.E. BARKWORTH, K.M. CAPELS, S. LONG AND M.B. PIEP (eds.), Magnoliophyta: Commelinidae (in part): Poaceae, Part 2. Flora of North America North of Mexico. Volume 25. Oxford University Press, New York. 65105.

PETERSON, P.M. AND J. VALDES-REYNA. 2005. Eragrostis (Poaceae: Chloridoideae: Eragrostideae: Eragrostidinae) from north eastern Mexico. Sida 21: 1365-1420.

PETERSON, P.M., R.J. SORENG, G. DAVIDSE, T. FILGUERIAS, F.O. ZULOAGA AND E. JUDZIEWICZ. 2001. Catalogue of New World grasses (Poaceae): II. subfamily Chloridoideae. Contr. U.S. Natl. Herb. 41: 1-255.

PETERSON, P.M. AND VEGA SANCHEZ. 2007. Eragrostis (Poaceae: Chloridoideae: Eragrostideae: 
Eragrostidinae) of Peru. Ann. Missouri Bot. Gard. 94: 745-790.

PETERSON, P.M. 2008. Eragrostis (Poaceae: Chloridoideae: Eragrostideae) in Colombia. J. Bot. Res. Inst. Texas 2 (2): 875-916.

PETERSON, P.M., R.D. WEBSTER AND J. VALDESREYNA. 1997. Genera of New World Eragrostideae (Poaceae: Chloridoideae). Smithsonian Contr. Bot. 87: $1-50$.

PETERSON, P.M., R.J. SORENG, S.M. PHILLIPS AND J.H. WIERSEMA. 2018. Proposal to reject the name Poa amabilis (Eragrostis amabilis) (Poaceae). Taxon 67(3): 644-645.

PHILlIPS, S.M. 1995. Poaceae (Gramineae). In: I. HEDBERG AND S. EDWARDS (eds.), Flora of Ethiopia and Eritrea 7:122. National Herbarium, Addis Ababa and Department of Systematic Botany, Uppsala.

PIENAAR, R. De V. 1955. The chromosome numbers of some indigenous South African and introduced Gramineae. In: MEREDITH, H.D. (ed.), Grasses and Pastures of South Africa. Central News Agency Ltd., Johannesburg. pp. 551-570.

POHL, R.W. 1980. Family 15. Gramineae. In: BURGER, W.C. (ed.), Flora Costaricensis. Fieldiana, Bot. 4: 1608.

PORTAL, R. 2002. Eragrostis de France et de l'Europe occidentale. Vals près Le Puy.

REEDER, J.R. 1977. Chromosome numbers in western grasses. Amer. J. Bot. 64:102-110.

ROSS, R. 1966. The generic names published by N.M. von Wolf. Acta Bot. Neerl. 15: 157.

ROY, G.P. 1976. The genus Eragrostis P. Beauv. in Rajasthan. Bull. Bot. Surv. India 18(1-4): 102-108.

ROY, G.P. 1984. Grasses of Madhya Pradesh. Botanical Survey of India, Calcutta.

SCHOLZ, H. 2000. In: S. CAFFERTY, C.E. JARVIS AND N.J. TURLAND, Typification of Linnaean plant names in the Poaceae (Gramineae). Taxon 49: 256.

SHANTHAMMA, C. AND K.N. NARAYAN. 1976-1977.
Studies of Poaceae (Gramineae ). J. Mysore Univ., Sect. B. 27: 302-305.

SHUKLA, U. 1996. The Grasses of North-Eastern India. Scientific Publishers, Jodhpur.

SOKOLOVSKAYA, A.P. AND O.S. STRLKOVA. 1969. In: FEDOROV, A.N.A. (ed.), Chromosome Numbers of Flowering Plants. Academy of Science of the V.L. Komorov Botanical Institute, U.S.S.R.

SREEKUMAR, P.V. AND V.J. NAIR. 1991. Flora of Kerala - Grasses. Botanical Survey of India, Calcutta.

STAPF, O. 1896. Eragrostis. In: HOOKER, J.D. (ed.), The Flora of British India. Vol. 7. L. Reeve \& Co., London. pp. 312-327.

STEUDEL, E.G. 1840. Nomenclator Botanicus. ed. 2, 1. 562-563, 565. Cotta, Stuttgart, Tübingen.

TATEOKA, T. 1953. Karyotaxonomic studies in Poaceae. I. Rep. (Annual) Natl. Inst. Genet. 4: 45-47.

TATEOKA, T. 1954. Karyotaxonomic studies in Poaceae. II. Rep. (Annual) Natl. Inst. Genet. 5: 68-69.

TATEOKA, T. 1965. Chromosome numbers of some grasses from Madagascar. Bot. Mag. 78: 306-311.

TATEOKA, T. 1967. In: IOPB. Chromosome Number Report XIV. Taxon 16: 552-571.

TOVAR, O. 1993. Las Gramineas (Poaceae) del Peru. Ruizia 13: 1-480.

TRINIUS, C.B. 1830. Graminum genera. Mém. Acad. Imp. Sci. St.-Pétersbourg, Sér. 6, Sci. Math. 1: 397, 405, 409, 411.

UMAMAHESHWARI, P. AND P.DANIEL. 1998. Eragrostis amabilis (L.) Wight \& Arn. (Poaceae) and its varieties. J. Econ. Taxon. Bot. 22(1): 211-216.

UNIYAL, B.P., B. BALODI AND B. NATH. 1994. The Grasses of Uttar Pradesh - A Checklist. Bishen Singh Mahendrapal Singh, Dehradun.

VELDKAMP, J.F. 2002. Revision of Eragrostis (Gramineae, Chloridoideae) in Malesia. Blumea 47: 157-204.

VELDKAMP, J.F. THOIBA KOTTEKKATTU AND A.K. PRADEEP. 2017. Eragrostis paniciformis (Poaceae): A 
new record for Asia from Kerala, India. Rheedea 27(1): $46-49$.

VIGNOLO, F. 1904. Sul valore sistematica della Poa cilianensis All. (1785). Malpighia 18: 380.

VIVEK, C.P., G.V.S. MURTHY AND V.J. NAIR. 2012. A new species Eragrostis henryi (Poaceae: Eragrostideae) from Tamil Nadu, India. Nelumbo 54: 9-12.

VIVEK, C.P., G.V.S. MURTHY AND V.J. NAIR. 2013a. Eragrostis nilgiriensis (Poaceae): a new grass species from Nilgiri district, Tamil Nadu, India. Nordic J. Bot. 30: 700-703. doi: 10.1111/j.1756-1051.2012.01676.x.

VIVEK, C.P., G.V.S. MURTHY AND V.J. NAIR. 2013b. Eragrostis collinensis (Poaceae: Eragrostideae) - a new species from the hills of Kerala and Tamil Nadu. Indian J. Forest. 36(3): 401-404.

VIVEK, C.P., G.V.S. MURTHY AND V.J. NAIR. 2013c. Eragrostis jainii (Poaceae: Eragrostideae) a new species from Kerala, India. Nelumbo 55: 1-5.

VIVEK, C.P., G.V.S. MURTHY AND V.J. NAIR. 2013d. A note on Eragrostis rottleri Stapf (Poaceae) and its lectotypification. Nelumbo 55: 109-112.

VIVEK, C.P., G.V.S. MURTHY AND V.J. NAIR. 2013e. On the confusion in the identity of Eragrostis atrovirens, E. gangetica and E. nutans. Nelumbo 55: 94-101.

VIVEK, C.P., G.V.S. MURTHY AND V.J. NAIR. $2013 f$. Eragrostis minor Host var. rajasthanensis (Poaceae: Eragrostideae) - a new variety from Rajasthan, India. J. Econ. Taxon. Bot. 37(4): 717-719.
VIVEK, C.P., G.V.S. MURTHY, G. GNANASEKARAN, K.A.A. KABEER AND V.J. NAIR. 2015a. A study on the caryopses morphology of the grass genus Eragrostis in India. Nelumbo 57: 1-10.

VIVEK, C.P., G.V.S. MURTHY AND V.J. NAIR. 2015b. Lectotypification of Eragrostis maderaspatana. Nelumbo 57: 40-42.

VIVEK, C.P., G.V.S. MURTHY AND V.J. NAIR. 2015c. On the identity of Eragrostis dayanandanii (Poaceae) described from Tamil Nadu, India. Nelumbo 57: 4345.

VIVEK, C.P., G. GNANASEKARAN, G.V.S. MURTHY AND V.J. NAIR. 2016. Microhairs of Indian Eragrostis Wolf (Poaceae) and their taxonomic significance. Nelumbo 58: 48-56.

VIVEK, C.P., G.V.S. MURTHY AND V.J. NAIR. 2019. On the identity of seven interrelated species of Eragrostis (Poaceae: Chloridoideae) in India. Nelumbo 61(2): 1023.

VIVEK, C.P., L.J. SINGH AND G.A. EKKA. 2020. Taxonomy and distribution of Eragrostis cumingii (Poaceae: Chloridoideae) in India. Rheedea 29(4): 306309.

VOIGT, P., N. RETHMAN AND M. POVERENE. 2004. Lovegrasses. Warm seasons (C4) grasses. Agron. Monogr. 45: 1027-1055.

WOLF, N.M. VON 1776. Genera Plantarum ...: 23. Danzig.

YADAV, S.R. AND M.M. SARDESAI. 2002. Flora of Kolhapur District. Shivaji University, Kolhapur. 